LBL-36575

UC-411

\title{
Semiclassical Methods in Chemical Reaction Dynamics
}

\author{
Srihari Keshavamurthy \\ Ph.D. Thesis \\ Chemistry Department \\ University of California \\ and \\ Chemical Sciences Division \\ Lawrence Berkeley Laboratory \\ University of California \\ Berkeley, CA 94720
}

December 1994

\section{DISCLAIMER}

This report was prepared as an account of work sponsored by an agency of the United States Government. Neither the United States Government nor any agency thereof, nor any of their employees, makes any warranty, express or implied, or assumes any legal liability or responsibility for the accuracy, completeness, or usefulness of any information, apparatus, product, or process disclosed, or represents that its use would not infringe privately owned rights. Reference herein to any specific commercial product, process, or service by trade name, trademark, manufacturer, or otherwise does not necessarily constitute or imply its endorsement, recommendation, or favoring by the United States Government or any agency thereof. The views and opinions of authors expressed herein do not necessarily state or reflect those of the United States Government or any agency thereof.

This work was supported by the Director, Office of Energy Research, Office of Basic Energy Sciences, Chemical Sciences Division, of the U.S. Department of Energy under Contract No. DE-AC03-76SF00098.

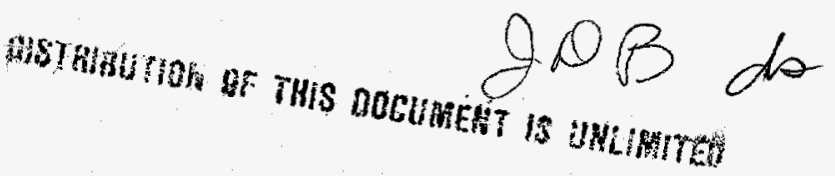




\section{DISCLAIMER}

Portions of this document may be illegible in electronic image products. Images are produced from the best available original document. 


\author{
Semiclassical Methods in Chemical Reaction Dynamics \\ by \\ Srihari Keshavamurthy \\ M. S. (Villanova University) 1989 \\ A dissertation submitted in partial satisfaction of the \\ requirements for the degree of \\ Doctor of Philosophy \\ in \\ Chemistry \\ in the \\ GRADUATE DIVISION \\ of the \\ UNIVERSITY of CALIFORNIA at BERKELEY
}

Committee in charge:

Professor William H. Miller, Chair

Professor Robert A. Harris

Professor Eugene D. Commins

1994 


\section{Abstract \\ Semiclassical Methods in Chemical Reaction Dynamics \\ by \\ Srihari Keshavamurthy \\ Doctor of Philosophy in Chemistry \\ University of California at Berkeley \\ Professor William H. Miller, Chair}

Semiclassical approximations, simple as well as rigorous, are formulated in order to be able to describe gas phase chemical reactions in large systems.

We formulate a simple but accurate semiclassical model for incorporating multidimensional tunneling in classical trajectory simulations. This model is based on the existence of locally conserved actions around the saddle point region on a multidimensional potential energy surface. Using classical perturbation theory and monitoring the imaginary action as a function of time along a classical trajectory we calculate state-specific unimolecular decay rates for a model two dimensional potential with coupling. The results are in good comparison with exact quantum results for the potential over a wide range of coupling constants.

We propose a new semiclassical hybrid method to calculate state-to-state Smatrix elements for bimolecular reactive scattering. The accuracy of the Van VleckGutzwiller propagator and the short time dynamics of the system make this method self-consistent and accurate. We also go beyond the stationary phase approximation by doing the resulting integrals exactly (numerically). As a result, classically forbidden probabilties are calculated with purely real time classical trajectories within this approach. Application to the one dimensional Eckart barrier demonstrates the accuracy of this approach.

Successful application of the semiclassical hybrid approach to collinear reactive scattering is prevented by the phenomenon of chaotic scattering. The modified 
Filinov approach to evaluating the integrals is discussed, but application to collinear systems requires a more careful analysis. In three and higher dimensional scattering systems, chaotic scattering is supressed and hence the accuracy and usefulness of the semiclassical method should be tested for such systems.

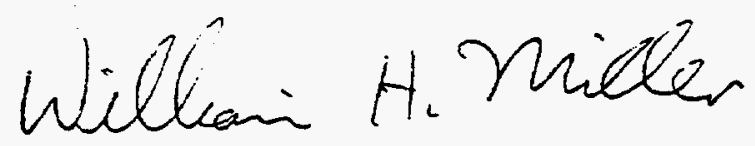


To my parents 


\section{Contents}

Table of Contents $\quad$ ii

List of Figures $\quad$ iv

1 General Introduction $\quad 1$

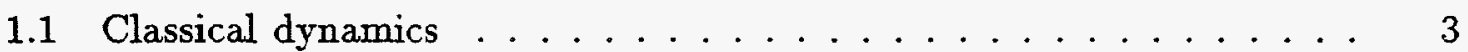

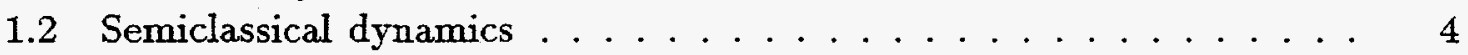

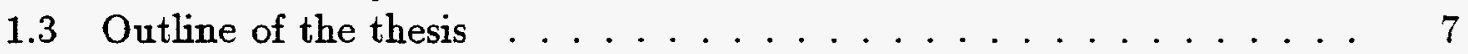

2 A Semiclassical Model for Tunneling 9

2.1 Introduction . . . . . . . . . . . . . . . . . 9

2.1.1 Earlier models ................... 11

2.2 The Semiclassical model . . . . . . . . . . . . . . 13

2.2.1 Determination of the tunneling action ........... 14

2.2.2 Determination of the tunneling time .......... 18

$2.3 k_{u n i}$ for a model potential . . . . . . . . . . . . 20

2.3.1 Choice of initial conditions . . . . . . . . . . 20

2.3.2 Results and Discussion . . . . . . . . . . . . . 22

2.4 Concluding remarks . . . . . . . . . . . . . . . . . . . . . . . . . . . . . . . .

2.4.1 Comments on the choice of $t_{n} \ldots \ldots . \ldots 27$

2.5 Appendix I: Classical perturbation theory ............ 29

2.5.1 Notations .................. . . . . 30

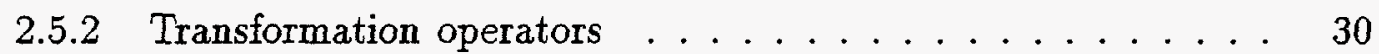

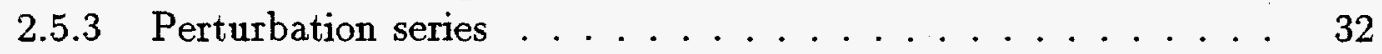

2.5.4 Simple example .................. 34

3 Semiclassical Scattering Theory 37

3.1 Introduction . . . . . . . . . . . . . . . 37

3.2 S-Matrix Kohn variational principle . . . . . . . . . . . . 41

3.3 Semiclassical approximation to $\Delta S_{\mathbf{n}^{\prime} \alpha^{\prime}, \mathbf{n} \alpha}(E) \ldots \ldots \ldots$

3.4 Application to a model potential . . . . . . . . . . . . . . . 48

3.4.1 Results and discussion . . . . . . . . . . . . . . 52 
3.5 Concluding remarks . . . . . . . . . . . . . . . 55

3.6 Appendix I: Analysis of the singularity in the integrand of Eq. (3.28) 57

3.7 Appendix II: Direct semiclassical approach to thermal rate constant $k(T)$-challenges and a new transition state result . . . . . . 59

3.7.1 A new semiclassical TST result for $k(T) \ldots \ldots \ldots \ldots 63$

4 Application to collinear $A+B C$ reactions 64

4.1 Introduction . . . . . . . . . . . . . . . . . 64

4.2 The Jacobi Hamiltonian . . . . . . . . . . . . . . . . 65

4.3 The semiclassical S-matrix . . . . . . . . . . . . . 66

4.3.1 Choice of the trial wavefunctions . . . . . . . . 67

4.3.2 Evaluating the Jacobian determinant . . . . . . . . 68

4.4 Behaviour of the integrand . . . . . . . . . . . 70

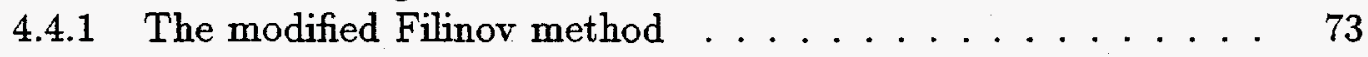

4.4 .2 Eckart barrier revisited . . . . . . . . . . . . 76

4.4.3 Modified Filinov approach to collinear systems . . . . . . . 79

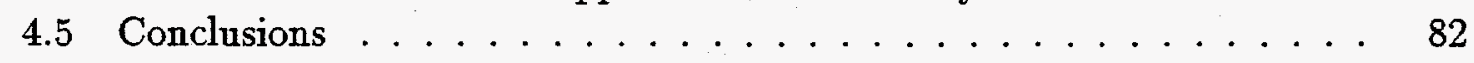

$\begin{array}{lc}\text { Bibliography } & 84\end{array}$ 


\section{List of Figures}

$2.1 \bar{J}_{F}(t)$ along a classical trajectory . . . . . . . . . . . . . 19

$2.2 k_{u n i}$ for specific initial reactant states, $c=0 a u \ldots \ldots$

$2.3 k_{u n i}$ for specific initial reactant states, $c=0.001$ au . . . . . . 24

$2.4 k_{u n i}$ for specific initial reactant states, $c=0.005 a u \ldots 25$

$2.5 \bar{J}_{F}(t), E$ and $E_{\text {pert }}$ along a classical trajectory . . . . . . . . . 28

3.1 Integrand as a function of $p_{1} \ldots \ldots \ldots \ldots \ldots$

$3.2 P(E)$ as a function of $E$ for $\lambda=2.5 \mathrm{au}$ and $x_{0}=5.0 \mathrm{au} \ldots \ldots$

3.3 Variation in $P(E)$ as a function of $x_{0} \ldots \ldots \ldots \ldots$

3.4 Summary of the difficulties involved in a direct semiclassical calculation

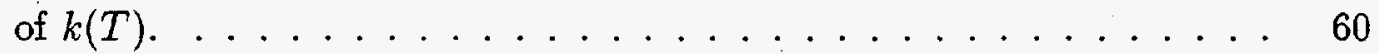

4.1 Determining the Jacobian determinant along a trajectory. . . . . . . 69

4.2 Typical plot of the imaginary part of the integrand as a function of $P_{R}^{\alpha} .71$

4.3 Typical plot of the imaginary part of the integrand as a function of $P_{R}^{\alpha}$ near a R-NR boundary. . . . . . . . . . . . . . 72

4.4 Three different kinds of reactive trajectories. . . . . . . . . . . 74

4.5 Integrand as a function of $p$ for various values of $\mathrm{b}$. . . . . . . . 78

4.6 Integrand as a function of $P_{R}^{\alpha}$ for various values of b. . . . . . . . 81 


\section{Acknowledgments}

I have been extremely fortunate to have had this chance to do my graduate research at Berkeley. I cannot begin to describe the influence both scientific and personal that two people have had during my stay here. My reseach advisor, Prof. William H. Miller, has been infinitely patient and encouraging through all these years. Among other things, Bill has taught me how to do good and useful science and his infectious style has had a great impact on my scientific outlook. Bill's insights into semiclassics have never ceased to amaze me and I have, hopefully, learnt a lot from all those discussions with him. Prof. Robert G. Littlejohn has been a source of inspiration to me ever since I got here at Berkeley. I still remember his lectures on classical dynamics during my first year. The style, content and clarity of his lectures have left a permanent mark on the way I understand classical mechanics. Over the years Robert has taught and shared with me his deep insights into classical and semiclassical dynamics. He always found time to discuss interesting semiclassical issues with me including some of my ideas which were absolutely crazy. I am indebted to both Bill and Robert for all the support and encouragement they have given me over my graduate career. They have provided me with lofty standards of research and teaching which $I$ aspire to achieve during my scientific career.

The great thing about being at Berkeley is the opportunity to listen and learn from the masters. I have been very lucky to have the pleasure of listening to some very good lectures. Prof. Commins' lectures on Quantum mechanics, Prof. Zumino's lectures on General Relativity, Prof. Alvarez's lectures on Quantum Field theory and Prof. Weinstein's lectures on classical and semiclassical dynamics are a few of those that I have learnt a great deal from and will cherish forever. I also thank Prof. Harris for all the interesting discussions and for providing me with an opportunity to teach his class-an assignment that I really enjoyed doing.

A big part of my graduate career was the interaction with the Miller group members. I appreciate their patience with all those blackboard sessions, cafe strada 
trips and various other idiosyncracies of mine. They have really made me feel at home. I am especially grateful to Dan Gezelter for asking me the questions about knotted periodic orbits and the relation between symmetry and integrability-questions which led me to study a lot of interesting topics. Dan also made all my computer problems disappear for which I thank him profusely. A lot of my all-nighters would have been dull and unproductive if it weren't for Ward Thompson's presence and his ability to discuss on topics ranging all the way from the NFL to the DVR-ABC. I would like to thank Prof. Nancy Makri for all the help she provided me with my first research project. I have enjoyed numerous interesting discussions with the Miller group postdocs, especially Dr. Gerrit C. Gröenenboom, Dr. Gerhard Stock, Dr. Peter Saalfrank and Dr. Uri Peskin. Thanks are also due to the rest of the Miller group past and present: Prof. Claude J. Leforestier, Dr. Tamar Seideman, Dr. Uwe Manthe, Dr. Agathe Untch, Dr. Yan-Tyng Chang, Dr. Lionel F. Gaucher, Dr. Scott M. Auerbach, Dr. Rigoberto Hernandez, Bruce W. Spath, Bill Poirier and Sean Sun. It is a pleasure to thank Mrs. Cheryn Gliebe for her moral support and taking care of all the administrative details.

During my stay here, I have been fortunate to have some very special friends. Thanks to Zoran Kurtovič for being such a great friend for all these years, Jim Morehead for patiently listening to my semiclassical mumblings, Dr. Kevin Leung, Raymond Yee and Felix Csjaka for many hours of stimulating discussions on a lot of random but interesting topics, Dr. Eric M. Hiller for telling me all the subtelties about Berry's phase and finally Dr. John N. Gehlen for help with the thesis and all those exciting hoops sessions.

This acknowledgment would be incomplete without mentioning the people responsible for my arrival at Berkeley. Profs. J. R. Delavega, O. G. Ludwig, and A. J. Phares have been great teachers and friends for all these years and I appreciate their interest in my scientific progress. Prof. T. K. Krishnamurthy played a key role during my undergraduate years in India and sparked my interest in theoretical chemistry through his impeccable lectures.

I have been extremely fortunate to have such great parents. My father and mother have been infinitely loving and are responsible to a large extent for what I am 
today. To them I dedicate this thesis with all my love and respect. As for my uncle and aunt Dr.and Mrs. M. J. Narasimha, it is not possible to thank them enough for all their hospitality towards me over these years at Berkeley. They have been very kind and supportive and were there for me in my hours of need. Finally, Suma has been a constant source of inspiration for me and it is hard to imagine life without her love and encouragement. I appreciate her patience during these thesis writing months.

This research was supported by the Director, Office of Energy Research, Office of Basic Energy Sciences, Chemical Sciences Division of the U.S. Department of Energy under Contract No. DE-AC03-76SF00098. 


\section{Chapter 1}

\section{General Introduction}

Chemical reaction dynamics is a fascinating field, full of complexities and surprises. As chemists we are interested in understanding chemical reactivity from first principles. There are various different levels of detail to understanding chemical reactivity, ranging from broad empirical guidelines to the description based on fundamental interactions at the atomic level. On the experimental side, we now have the capability to probe and study chemical reactions in a very detailed fashion. Recent progress in experimental techniques like molecular beam methods[38] and high resolution spectroscopies[39] have led reseachers to measure lifetimes, reaction rates and other interesting quantum state-specific properties of fairly complicated molecules. With the advent of ultrashort laser pulses[84] it is also possible to study the realtime evolution of specific initial states. These experimental advances have provided a challenge and motivation for the theoreticians to understand chemical reactions at the microscopic level. The task for a theoretical chemist, in my opinion, is to formulate models for chemical reactions which lead to valuable insights into the nature of chemical reactivity and provide a clear explanation for the experimental results.

In contrast to the experimental advances, theoretical description of chemical reaction dynamics at similar levels of detail is not yet well developed. In principle, at the fundamental level, we can certainly write down the Hamiltonian for a complicated system and thus the Schrödinger or Hamilton's equation describing the dynamics. However, it is an entirely different issue to solve these equations in a rigorous fashion 
due to the fact that in any chemical system we have a large number of strongly coupled degrees of freedom. Solving the Schrödinger equation rigorously for several strongly coupled degrees of freedom is still out of reach of our current computational capabilities. In fact the best we can do is a four atom (six degrees of freedom) reaction[44]. In addition, we need accurate $a b$ initio potential surfaces in order to perform dynamics in a reliable way. Present levels of sophistication in electronic structure theory cannot provide accurate potential surfaces for more than a few atom systems. Thus, it becomes very important to consider approximate approaches to develop potential surfaces and solving for the dynamics of a complicated chemical system.

There has been a flurry of activities in the recent years in developing efficient approximate methods to solve the Schrödinger equation for both gas phase and condensed phase reactions. Considerable progress has been made in the theory of reactive scattering[41] in order to compute state-specific transition probabilities. The S-matrix Kohn variational principle[42] formulation of reactive scattering represents a significant advance in our capabilities to calculate state-specific observables for gas phase reactions. For the calculation of averaged observables like the thermal rate constants and cummulative reaction probabilities, there are many direct approaches including the path integral method [5, 74] and methods based on time dependent[47] and time independent $[44,46]$ scattering theory. Path integral methods, based on the influence functional idea[62,85, 12], also have been useful in describing reactions in condensed phases. Despite these numerically attractive and ingenious approaches to solving the Schrödinger equation, we still cannot go beyond a few atom systems. One of the main bottlenecks has to do with the speed and memory limitations of our current computing capabilities. It is clear that computers will continue to grow in speed and memory size making it possible to provide a realistic description of larger molecular systems, but we are always interested in carrying out reliable calculations that are beyond the capabilities of a fully rigorous quantum treatment. The question is then, are there ways to gain insights into the dynamics of a chemical reaction which are computationally straightforward, qualitative and quantitative. From a numerical and intutive standpoint, classical mechanics offers such a framework to study reactions of 
complicated chemical systems.

\subsection{Classical dynamics}

It is well known that classical trajectory calculations are feasible for truly complex molecular systems. For example, in the description of reactions in condensed phases molecular dynamics simulations play a very important role[86] due to the fact that the numerical effort in solving the coupled first order Hamilton's equations of motion scales linearly with the number of degrees of freedom. Moreover, recent advances in our understanding of the foundations of classical mechanics $[19,87,88,89,8]$ has provided a variety of methods to analyze and gain insights into the classical dynamics of complicated systems. Considerable amount of work has been done in understanding the structure of phase space of complicated dynamical systems leading to insights on bimolecular and unimolecular reactions[79, 80]. Researchers are realizing that clarifying the rich phase space structure of a complicated system can yield valuable information about the corresponding underlying dynamics[90, 94]. This is still a very active area of research. Nevertheless, a naive use of classical mechanics cannot describe processes which are of importance in chemical reactions. An important example is tunneling, which dominates the rates at low temperatures when the reaction involves light atoms such as hydrogen $[1,4]$.

Let me expand a little on the use of the words naive classical mechanics. Ordinarily, we deal with classical trajectories in real time defined via the Hamilton's variational principle[19]

$$
\delta \mathcal{S}[\mathbf{q}(t)] \equiv \delta \int_{0}^{t} d t^{\prime} \mathcal{L}\left[\mathbf{q}\left(t^{\prime}\right), \dot{\mathbf{q}}\left(t^{\prime}\right), t^{\prime}\right]=0,
$$

where $\mathcal{S}$ and $\mathcal{L}$ represent the action functional and the Lagrangian of the system respectively. In real time $t$ classical trajectories cannot evolve from one phase space region to another equivalent but disconnected phase space region. For example, consider the simple symmetric double well potential. It is easy to see in this case that for energies below the barrier height, the phase space is made up of energy manifolds which are topologically disconnected. Thus, a real time classical trajectory evolving 
on one of the energy manifolds cannot continously evolve into the other equivalent energy manifold. Hence, tunneling cannot be accounted for in this version of classical mechanics. However, notice that in Eq. (1.1) there is no requirement that $t$ be real. In fact, interestingly, the consequences of relaxing the constraint of real $t$ leads to tunneling in this analytically continued version of classical mechanics[91]. Incidentally, the analysis of classical trajectories in complex time is linked to certain interesting conjectures about the integrability of the corresponding classical Hamiltonian $[92,88]$. In this thesis, we will be concerned not with analytically continued classical mechanics, but with semiclassical extensions of the naive classical mechanics in ordex to properly account for classically forbidden processes in chemical reactions.

There are other effects like quantum interference, zero point energies which are again not explicitly present in the naive classical mechanics. An elegant approach to build in these effects, keeping intact all of the usual adavntages of classical mechanics, is to consider families of classical trajectories as opposed to a single classical trajectory $[9,93]$. Each one of the classical trajectories has assosciated with it an amplitude and a phase which are classical quantities and the idea of quantum superposition is introduced. Thus, observables are calculated by summing up the contributions from each individual trajectory at the amplitude level. By explicitly including superposition, we are guaranteed to treat essentially all quantum effects like tunneling, interference, quantization and selection rules[9]. We are thus naturally lead to semiclassical dynamics in order to construct the precise form of these amplitudes and phases.

\subsection{Semiclassical dynamics}

Semiclassical methods are very general and powerful techniques to study the asymptotic properties of the solutions to differential or integral equations $[93,60$, $24,89,94]$. In fact, for any system which can support solutions in the form of a rapidly varying phase with a slowly modulated amplitude we can use semiclassical approximations in order to gain insights about the system. For example, semiclassical methods are used in such diverse fields like wave propagation in atmosphere and 
interacting seismic waves.

As far as this thesis is concerned we will be interested in semiclassical approximations to the non-relativisitc Schrödinger equation. For this case, the semiclassical methods yield asymptotic solutions to the wavefunction in the limit that the relevant classical actions are much larger than compared to the fundamental unit of action $\hbar$. Semiclassical application to bound state problems has a very long and illustrious history. For example, semiclassical anaysis of the energy eigenfunctions leads to the celebrated Bohr-Sommerfeld or Einstein-Brillouin-Keller[23] quantization of the energy levels for an integrable system via

$$
\oint_{\mathcal{C}_{j}} \mathbf{p} \cdot d \mathbf{q}=2 \pi \hbar\left(n_{j}+\frac{\mu_{j}}{4}\right)
$$

where $\mathcal{C}_{j}$ is a closed contour on an invariant torus, $n_{j}$ is the quantum number and $\mu_{j}$ is the Maslov index[60]. Quantization of integrable systems is a very well established field. The quantization of non-integrable systems is a very different story. Here there are no invariant tori in phase space and the relevant objects are periodic orbits in phase space as shown in the seminal work by Gutzwiller[89, 95]. However, the problem of quantization of non-integrable systems is not a solved problem due to the fact that the Gutzwiller approach is not well behaved. Recent progress in this area has been pioneered by many people including Artuso[96], Littlejohn[97], Wintgen[98] and Berry[99]. The problem also has to do with understanding the consequences of non-integrability in the corresponding quantum system. This is still a very interesting and active field of research.

On the other hand, an important contribution to semiclassical analysis to molecular collisions was made by Ford and Wheeler[100] who showed how elastic scattering of atoms could be described semiclassically. Later Miller[9] generalized the ideas and provided a very elegant and practical approach to applying semiclassical methods to more complicated systems which could undergo inelastic and reactive collisions. This classical S-matrix theory shows how to calculate the state-to-state S-matrix elements for reactive collisions. The only difficult feature of this theory is that taking into account classically forbidden processes like tunneling involved complex valued classical trajectories. Nevetheless, in a series of seminal papers, Miller 
and George[11] demonstrated the accuracy of the theory by analytically continuing classical mechanics. Despite the success, it was realized that application to truly large systems was prohibitively difficult.

Thus far we have concentrated on semiclassical approximations in the energy representation. It is also equally possible to perform the analysis in the time domain. The main contribution in this area was by Van Vleck who derived the semiclassical approximation to the propagator and Gutzwiller who later derived a more accurate version of Van Vleck's results which was valid for longer times[64]. The resulting expression is the Van Vleck-Gutzwiller approximation[64]

$$
\begin{aligned}
\left\langle\mathbf{x}_{2}\left|\exp \left(-\frac{i \hat{H} t}{\hbar}\right)\right| \mathbf{x}_{1}\right\rangle \approx\left(\frac{1}{2 \pi i \hbar}\right)^{F / 2} \sum_{k} \mid & \left.\operatorname{Det}\left(\frac{\partial^{2} \mathcal{S}_{k}\left(\mathbf{x}_{2}, \mathbf{x}_{1} ; t\right)}{\partial \mathbf{x}_{2} \partial \mathbf{x}_{1}}\right)\right|^{1 / 2} \\
& \times \exp \left(\frac{i \mathcal{S}_{k}\left(\mathbf{x}_{2}, \mathbf{x}_{1} ; t\right)}{\hbar}-\frac{i \pi \mu_{k}}{2}\right)
\end{aligned}
$$

where $\mathcal{S}_{k}$ is the classical action for the $k^{\text {th }}$ classical trajectory, $F$ is the number of degrees of freedom and $\mu_{k}$ is the Maslov index (different from the one in Eq. (1.2)). However, the work of Berry et al.[68] raised doubts about the accuracies of semiclassical approximations for long times when the classical dynamics was irregular. They linked the failure of semiclassical approximations to the development of complicated phase space structures and demonstrated it by applying to the quartic oscillator problem. They also provided some estimates of the time scales for which the semiclassical propagation could be usefully accurate. This feature of the semiclassical propagator seemed to limit its applicability until recently when Heller and Tomsovic[58] demonstrated accuracy of the approximation to much longer times then that implied by Berry et al. It was shown that the semiclassical propagator provided very accurate results for strongly chaotic systems even when the corresponding phase space had developed incredibily complicated structures. Later, application [58, 59] to mixed systems showed similar accuracies and once again the Van Vleck-Gutzwiller approximation was rejuvenated. In a series of papers, Heller and coworkers have demonstrated the accuracy and usefulness of the semiclassical propagator. In retrospect, the analysis of Berry et al. was not quite general as the quartic oscillator example is not the 
typical case due to some of the special properties of this system.

To summarize, the semiclassical analysis for bound or unbound systems allows us to construct the amplitude and phase assosciated with each classical trajectory. From Eq (1.3), the phase is simply given by the classical action which satisfies the Hamilton-Jacobi equation $[19,88]$ and the amplitude is related to the stability of nearby classical trajectories and satisfies the amplitude transport equation. The amplitude also plays the role of a classical probability. In addition to providing physical insights into the system, the semiclassical approximations can be usefully accurate over a wide variety of situations ranging from completely integrable to strongly chaotic systems. The next few chapters in this thesis will demonstrate the usefulness of the semiclassical approximations for molecular systems.

\subsection{Outline of the thesis}

The main theme of this thesis is concerned with semiclassical analysis of general multidimensional systems. It is precisely these systems which are of interest to us in reaction dynamics due both to their richness and complexity. The problem of finding good conserved actions is intimately linked to the problem of integrability or non-integrability of these strongly coupled multidimensional systems. Even for a non-integrable system it is possible to find locally conserved actions around stationary points on the corresponding potential surface. It is shown in chapter 2 that these locally conserved actions can provide us with a simple but accurate semiclassical model for multidimensional tunneling.

In chapter 3 we make use of the recent ideas put forward by Heller et al. to formulate a semiclassical hybrid model for reactive scattering. Again, a better understanding of the underlying phase space allows us to go beyond stationary phase approximation and obtain the state-to-state S-matrix elements. It is shown that within this approach classically forbidden processes are accounted for via purely real time classical trajectories.

Finally, in chapter 4 we apply the hybrid approach to collinear reactive scattering and discover a significant bottleneck due to chaotic scattering. Some sug- 
gestions are offered to alleviate the problem. 


\section{Chapter 2}

\section{A Semiclassical Model for Tunneling}

\subsection{Introduction}

Tunneling plays a very dominant role in chemical reactions at low temperatures, especially reactions which involve significant motion of light atoms[1] . Isomerization and unimolecular decay are two of the important types of phenomena which are quite common in chemistry. In the case of isomerization reactions tunneling leads to energy splittings of the degenerate states. As far as unimolecular decay reactions are concerned, tunneling gives rise to finite lifetimes for the metastable states through coupling to the continuum[2]. Both the energy splittings and the lifetimes are experimentally measurable quantities and indeed experiments have been performed on molecules like malonaldehyde and formaldehyde in order to obtain detailed information about the tunneling dynamics of these molecules[3]. Tunneling also contributes significantly near and below the threshold region of bimolecular reactions involving light atoms, for example

$$
H_{2}+X \rightarrow H+H X
$$

There are many such examples, spanning diverse fields, where tunneling plays an important role in determining the low temperature behaviour of the system of interest[4]. 
It is thus important for theoreticians to accurately predict the effect of tunneling on rates of chemical reactions.

Most of the reactions of interest in chemistry involve large molecules. This leads to the fact that the tunneling degree of freedom is coupled to the rest of the degrees of freedom of the molecule. For example, intramolecular hydrogen atom transfer reaction in malonaldehyde, which is a nine atom system, would involve all of the $3 N-6(=21)$ degrees of freedom of the molecule. An exact quantum calculation on such a large system is out of reach of the current theoretical methods. At this stage one makes an approximation, albeit a good one, by considering only a few modes strongly coupled to the large amplitude tunneling degree of freedom. However, all of the recent progress in exact quantum methods still cannot account for more than six degrees of freedom. Feynman path integral approaches certainly seem promising in extending the capabilities of exact quantum methods due to the recent advances in the Monte Carlo methods for evaluation of the path integrals[5]. There are a host of approximate quantum approaches like the MulticonfigurationTime Dependent-Selfconsistent Field (MC-TDSCF) method[6] which can be applied to larger systems than fully quantum approaches but they are still limited. There have been considerable efforts to incorporate tunneling corrections to transition state theory expressions for thermal rate constants[7]. However, they are not applicable to more general dynamical phenomena. On the other hand, classical trajectories are easily computed for systems with a very large number of degrees of freedom[8]; but naive classical mechanics does not allow tunneling from one classically allowed region of phase space to another. In order to exploit the attractive features of classical mechanics in situations involving classically forbidden dynamics one has to consider semiclassical extensions to classical mechanics.

There already exist semiclassically rigorous theories for multidimensional tunneling, for example, the classical S-matrix theory[9] and the instanton (periodic orbit in imaginary time) model[10]. However, in both cases one needs to calculate classical trajectories in complex time. Miller and George[11] have analytically continued classical mechanics and demonstrated its accuracy in the case of the collinear $\mathrm{H}+\mathrm{H}_{2}$ reactions. Analytic continuation of classical mechanics to complex times is 
a very rigorous way of approaching classically forbidden dynamics, but it is numerically very challenging. In particular, application to higher dimensional systems is not at all straightforward. The instanton model has gained considerable popularity for describing tunneling in both gas phase and condensed phase systems[12]. But the instanton corresponds, dynamically, to a zero kinetic energy trajectory on the upsidedown potential - which makes it effective to describe only the ground state tunneling dynamics. For higher energy states, the instanton model does not perform as well. Again, one needs to calculate periodic orbits in imaginary time which becomes difficult in situations where the number of degrees of freedom is very large. In addition, if the potential has a non-quadratic minimum then the instanton method is inapplicable[37]. One should also note that in case of one degree of freedom, the standard Wentzel-Kramers-Brillouin (WKB) approximation [13] works quite well, however a satisfactory generalization of WKB theory for multidimensional tunneling is still lacking[37]. Hence, it is of paramount importance to consider simple semiclassical models to incorporate tunneling in classical trajectory simulations. It is important to realize that, in coming up with simple models at the price of sacrificing a certain amount of rigour inherently leads to ad hoc models.

\subsubsection{Earlier models}

There has been a lot of work in the literature dealing with simple models for multidimensional tunneling[14]. Most of these simple models involve running classical trajectories in the allowed region of phase space and at certain times (tunneling times, $t_{n}$ ) computing the probability of tunneling, instantaneously in real time, to another allowed region of phase space. They are similar in spirit to the Tully-Preston surface hopping model[15] for electronically non-adiabatic processes.

Central to all these models is the determination of

- Tunneling times, $t_{n}$

- Tunneling path, $\Gamma_{n}$

- Action, $\theta_{n}$, along $\Gamma_{n}$ to determine the tunneling amplitude, $e^{-\theta_{n}}$.

It has to be emphasized that the path, $\Gamma_{n}$, is a nondynamical path. In other words, $\Gamma_{n}$ 
is not a classical trajectory obtained by solving for the Hamilton's equations of motion in the classically forbidden region, but some a priori path picked in the coordinate space. For example, it could be a straight line path connecting one point in the classically allowed region to another classically allowed region. Assosciated with $\Gamma_{n}$ is also a tunneling direction $\mathbf{e}_{n}$. The trajectory is then allowed to tunnel along $\Gamma_{n}$ in the direction $\mathbf{e}_{n}$ every time the component of the trajectory momentum $\mathbf{p}$ along $\mathbf{e}_{n}, \mathbf{p .} \mathbf{e}_{n}$, experiences a classical turning point (i.e., goes through zero). Thus, as the classical trajectory evolves, at times $t_{n}$ an amplitude for tunneling is calculated and a certain amount of probability "leaks out" of the classically allowed region. The net tunneling amplitude

$$
A_{n e t}(t)=\sum_{n} h\left(t-t_{n}\right) e^{-\theta_{n}},
$$

where

$$
\theta_{n}=\frac{1}{\hbar} \operatorname{Im} \int_{\Gamma_{n}} \mathbf{p}(\mathbf{q}) \cdot d \mathbf{q},
$$

is calculated as a function of time. $h(\eta)$ is the usual step function (=1 if $\eta>0$ and $=0$ if $\eta<0) . A_{\text {net }}(t)$ is then averaged over appropriate initial conditions to obtain the average net tunneling amplitude $\left\langle A_{n}(t)\right\rangle$. The unimolecular decay rate or energy splittings are related to the time derivative of $\left\langle A_{n}(t)\right\rangle$. These models have been used to calculate tunneling splittings and decay rates with a simple straight line approximation for $\Gamma_{n}[16]$. The results are quite impressive even when the coupling between the large amplitude tunneling mode and the rest is so large as to change the results by 2 orders of magnitude.

The choice of $\Gamma_{n}$ is very critical to the model. A straight line approximation is the simplest possible and motivated by the fact that rigorous theories show that the optimum tunneling path is relatively straight in the tunneling region. However, one is left wondering if there are ways to allow for more general paths that would lead to better results at higher coupling constants. Unfortunately, attempts to generalize the straight line path to more general paths do not lead to better results. Worse yet, the results seem to deviate significantly from the exact results at even lower couplings than the straight line approximation[17]. The results are very sensitive to the particular path being chosen[18]. The expalnation for this is as follows: Suppose 
$\Gamma_{n}$ were a dynamical path (i.e., projection of the exact classical trajectory in phase space on to the cofiguration space). Then, from well known results from classical mechanics[19], one can prove that

$$
\int_{\Gamma_{n}} \mathbf{p}(\mathbf{q}) \cdot d \mathbf{q}
$$

is only a function of the end points and does not depend on the path. If $\Gamma_{n}$ is a nondynamical path in configuration space, as is indeed the case with the path in the model, then the action will, in general, depend on the path. In addition, the straight line path is a one dimensional path which is relatively easy to specify in two dimensional cases. In $N>2$ dimensions the specification of the direction might not be easy let alone unique. Thus, one asks the question - Is it possible to come up with a semiclassical model, in the same spirit as the above model, where the tunneling times and actions are computed in a dynamically consistent fashion i.e., with no necessity of explicitly postulating a nondynamical path in order to calculate the action.

The next section describes such a model[20] which is simple and satisfies all the requirements. Application to a model two dimensional unimolecular decay potential and conclusions are described in subsequent sections. In particular, it is seen that the model has the capability of accurately predicting tunneling decay rates from specific initial reactant states over a wide range of system parameters.

\subsection{The Semiclassical model}

The starting point for this model is the same as that of the model described above in that we adopt a Tully-Preston like approach. The significant difference from the previous model[16] is that we adopt a different strategy to determine $\theta_{n}$ and $t_{n}$. In particular, our choice for $\theta_{n}$ and $t_{n}$ is dynamically consistent i.e., there is no need to specify, a priori, a tunneling direction, path or time. The motivation for this choice comes from earlier work[21] which showed that good i.e., locally conserved actions exist not only around potential minima but also around transition states. This allows us to write down a perturbative expression for the tunneling action as a function 
of the total energy and $F-1$ real actions determined in a dynamically consistent manner.

\subsubsection{Determination of the tunneling action}

Consider a cartesian, non-separable classical Hamiltonian in $F$ degrees of freedom

$$
H(\mathbf{p}, \mathbf{q})=\frac{\mathbf{p} \cdot \mathbf{p}}{2 m}+V(\mathbf{q}) .
$$

The Hamiltonian is said to be completely integrable[19] if $F$ time independent constants of motion, $\left\{f_{k}(\mathbf{p}, \mathbf{q}), k=1, \ldots, F\right\}$, exist which are mutually commuting in a Poisson bracket sense i.e.,

$$
\left\{f_{k}, f_{l}\right\} \equiv \frac{\partial f_{k}}{\partial \mathbf{p}} \frac{\partial f_{l}}{\partial \mathbf{q}}-\frac{\partial f_{k}}{\partial \mathbf{q}} \frac{\partial f_{l}}{\partial \mathbf{p}}=0
$$

where $k, l=1, \ldots, F$. In this case one can perform a canonical transformation to obtain the Hamiltonian purely as functions of $f_{k}, K\left(f_{1}, \ldots, f_{F}\right)$. The classical Hamilton's equations of motion take a very simple form

$$
\begin{aligned}
& \frac{d g_{k}}{d t}=\frac{\partial K}{\partial f_{k}} \\
& \frac{d f_{k}}{d t}=-\frac{\partial K}{\partial g_{k}}=0
\end{aligned}
$$

where $\left\{g_{k}(\mathbf{p}, \mathbf{q}), k=1, \ldots, F\right\}$ are the variables conjugate to $f_{k}$. As an example, consider the one dimensional harmonic oscillator Hamiltonian

$$
H(p, q)=\frac{p^{2}}{2 m}+\frac{m \omega^{2}}{2} q^{2},
$$

where $m$ and $\omega$ are the mass and frequency respectively. Performing the canonical transformation $(p, q) \rightarrow(J, \psi)$, where

$$
\begin{aligned}
q & =\sqrt{\frac{2 J}{m \omega}} \sin \psi \\
p & =\sqrt{2 m \omega J} \cos \psi,
\end{aligned}
$$

one obtains the new Hamiltonian

$$
K(J, \psi)=\omega J
$$


The variables $(J, \psi)$ are referred to as action-angle variables. The equations of motion in the $(J, \psi)$ variables are very simple and immediately integrated to give

$$
\begin{aligned}
& \psi(t)=\psi(0)+\omega t \\
& J(t)=J(0) .
\end{aligned}
$$

However, a generic multidimensional Hamiltonian is nonintegrable and there are no global constants of motion. Even if the Hamiltonian is integrable, analytically finding all the constants of motion is a non-trivial task. In addition, disentangling the canonical transformation to obtain the conserved quantities as functions of $(\mathbf{p}, \mathbf{q})$ is not an easy problem either. Thus, in order to keep the model practical we take a perturbative approach to calculate the conserved quantities. If we are dealing with an integrable system then the perturbation expansions have a finite radius of convergence. In the case of nonintegrable systems there are no global constants of motion and a perturbation calculation only gives locally conserved quantities ${ }^{1}$. Nevertheless, progress can be made by considering stationary points on the potential energy surface. Around these points it is possible, in a perturbative sense, to find action-angle variables in terms of which the Hamiltonian involves only the actions. For example, around a potential minimum one obtains the following well known result[22]

$$
\begin{aligned}
H(\mathbf{p}, \mathbf{q}) & \rightarrow H(\mathbf{J}, \boldsymbol{\psi}) \\
& =V_{0}+\sum_{k=1}^{F} \Omega_{k} J_{k}+\sum_{k \leq l=1}^{F} x_{k l} J_{k} J_{l}+\ldots,
\end{aligned}
$$

where $\left\{\Omega_{k}\right\}$ are the normal mode vibrational frequencies, and $\left\{x_{k l}\right\}$ are the anharmonic constants. In this case one quantizes the actions semiclassically

$$
J_{k}=\left(n_{k}+\frac{1}{2}\right) \hbar
$$

where $\left\{n_{k}\right\}$, the vibrational frequencies, are non-negative integers. This leads to the semiclassical Einstein-Brillouin-Keller (EBK) [23] vibrational energy levels[24]. The important point here is that a similar analysis holds around a saddle point on the potential surface as shown in an early work by Miller[21].

\footnotetext{
${ }^{1}$ Using the Kolmogoroff-Arnold-Moser (KAM) theorem we can make these statements more precise[19].
} 
Around a saddle point it is still possible to find local action-angle variables in terms of which the Hamiltonian is purely a function of the action variables. The difference from the analyis around a minimum is that one of the frequencies, $\Omega_{F}$ say, is pure imaginary. This corresponds to the reaction coordinate mode. It is easy to see that the corresponding action, $J_{F}$, is also pure imaginary and so are the anharmonic constants $x_{k F}, k \neq F$. Substituting

$$
\begin{aligned}
\Omega_{F} & =i \bar{\Omega}_{F}, \\
J_{F} & =i \bar{J}_{F}, \\
x_{k F} & =-i \bar{x}_{k F},
\end{aligned}
$$

where, $\bar{\Omega}_{F}, \bar{J}_{F}$ and $\bar{x}_{k F}$ are real, we obtain the equation corresponding to Eq. (2.12) for points around the saddle point as

$$
\begin{aligned}
E\left(\mathbf{J}^{\prime}, \bar{J}_{F}\right) & \equiv H\left(\mathbf{J}^{\prime}, \bar{J}_{F}, \boldsymbol{\psi}\right) ; \quad \mathbf{J}^{\prime} \equiv\left(J_{1}, \ldots, J_{F-1}\right) \\
& =\alpha\left(\mathbf{J}^{\prime}\right)-\beta\left(\mathbf{J}^{\prime}\right) \bar{J}_{F}-x_{F F} \bar{J}_{F}^{2}+\ldots,
\end{aligned}
$$

where

$$
\begin{aligned}
& \alpha\left(\mathbf{J}^{\prime}\right) \equiv V_{0}+\sum_{k=1}^{F-1} \Omega_{k} J_{k}+\sum_{k \leq l=1}^{F-1} x_{k l} J_{k} J_{l}, \\
& \beta\left(\mathbf{J}^{\prime}\right) \equiv \bar{\Omega}_{F}-\sum_{k=1}^{F-1} \bar{x}_{k F} J_{k} .
\end{aligned}
$$

Using the expansion (2.15) we can determine $\bar{J}_{F}$ as a function of the conserved total energy $E$ and $F-1$ real actions $\mathbf{J}^{\prime}$. At this juncture we assosciate $\bar{J}_{F}$ with the tunneling action as follows

$$
\theta\left(\mathbf{J}^{\prime} ; E\right)=\frac{\pi}{\hbar} \bar{J}_{F}\left(\mathbf{J}^{\prime} ; E\right) .
$$

The tunneling or transmission probability through the transition state region is given by

$$
P\left(\mathbf{J}^{\prime} ; E\right)=\frac{1}{1+e^{2 \theta\left(\mathbf{J}^{\prime} ; E\right)}} .
$$

Here we are interpreting $\theta\left(\mathbf{J}^{\prime} ; E\right)$ as a multidimensional generalization of the one dimensional WKB barrier penetration integral[24]

$$
\int p(q) d q=\int d q \sqrt{2 m[V(q)-E]} .
$$


With this interpretation, the tunneling probability in Eq. (2.18) is a uniformly valid[24] semiclassical result i.e, it is valid for energies above and below the barrier height. The inversion of the expansion (2.15) is simplified by keeping terms upto second order in $\bar{J}_{F}$. A quadratic equation is obtained which can be solved for $\bar{J}_{F}$ as a function of $\mathbf{J}^{\prime}$ and $E$

$$
\bar{J}_{F}\left(\mathbf{J}^{\prime} ; E\right)=\frac{2\left(\alpha\left(\mathbf{J}^{\prime}\right)-E\right)}{\beta\left(\mathbf{J}^{\prime}\right) \gamma\left(\mathbf{J}^{\prime}\right)},
$$

where

$$
\gamma\left(\mathbf{J}^{\prime}\right) \equiv\left(1+\sqrt{1+\frac{4 x_{F F}\left(\alpha\left(\mathbf{J}^{\prime}\right)-E\right)}{\beta^{2}\left(\mathbf{J}^{\prime}\right)}}\right) .
$$

Hence, we have determined the tunneling action where the path is a dynamical path, in a perturbative sense, in the full phase space of the system. Note that the actions are not globally conserved anymore but conserved only in a local sense.

It is worthwhile pointing out that in this model we can exploit the recent advances in $a b$ initio quantum chemistry to determine normal mode frequencies and anharmonic constants. Handy and coworkers have shown how the analytic second derivative methods of $a b$ initio quantum chemistry (including the effects of electron correlation) can be used to accurately determine the $\Omega_{k}$ 's and $x_{k l}$ 's[25]. Given a set of frequencies and anharmonicities for a molecule about the transition state, it is possible to calculate the tunneling probabilites through the transition state in order to determine microcanonical and canonical transition state theory rate constants. This particular model is then a transition state based theory. Application to several systems of interests like unimolecular decay reaction of deuterated formaldehyde and collinear $\mathrm{H}+\mathrm{H}_{2}$ reaction has yielded accurate results[26]. In our present model we are running classical trajectories combined with perturbation theory in order to calculate tunneling probabilities. Thus in additon to the frequencies and anharmonicities we also need a potential surface to run the classical trajectories ${ }^{2}$. In this fashion we are explicitly including the underlying complicated dynamics of the system.

\footnotetext{
${ }^{2}$ Obtaining a global potential energy surface for a multidimensional system is very difficult if not impossible. It can be argued that for this model based on locally conserved actions, only part of the potential, regions around transition states, would be necessary. There is a lot of interest in the chemical community to build up potentials based solely on force constant data. Perhaps for a large molecule this model can be applied without the need for a global potential surface.
} 
The results in this section have been derived by performing classical Lie transform[27] perturbation theory on our original Hamiltonian. We can quantize the actions $\mathbf{J}^{\prime}$ semiclassically as in Eq. (2.13) and interpret them as the quantum numbers for the states of the activated complex. Similar results can be obtained by taking the corresponding quantum Hamiltonian and performing canonical Van-Vleck perturbation theory[28] on the Hamiltonian. The details of the perturbation method are presented in the appendix to this chapter. However, in this model we want to be consistent by calculating everything as a function of the trajectory evolving in the classically allowed region of phase space.

\subsubsection{Determination of the tunneling time}

We make use of our locally conserved actions to determine the tunneling time. We run a classical trajectory in the allowed region of phase space under the exact multidimensional potential. The barrier action $\bar{J}_{F}$ is a function of the classical trajectory $(\mathbf{p}(t), \mathbf{q}(t))$ through the canonical transformation $(\mathbf{p}, \mathbf{q}) \rightarrow(\mathbf{J}, \boldsymbol{\psi})$. The time dependence of $\bar{J}_{F}$ is monitored along the exact classical trajectory. If $\bar{J}_{F}$ were globally conserved then we would see a trivial dependence on time i.e., $\bar{J}_{F}(t)=\bar{J}_{F}(0)$. However $\bar{J}_{F}$ is only locally conserved which results in the fact that it is approximately conserved in the vicinity of the saddle point and far from being conserved away from the saddle point. Figure 1.1 shows such a plot of $\bar{J}_{F}$ being conserved at certain times along the trajectory. The tunneling times are chosen to be that when $\bar{J}_{F}$ is locally conserved (stationary) in time.

This completes the description of the model. To summarize, one first perturbatively works out the locally conserved transition state action, $\bar{J}_{F}$, as a function of cartesian variables $(\mathbf{p}, \mathbf{q})$. Along the classical trajectory we monitor the values of $\bar{J}_{F}(t) \equiv \bar{J}_{F}(\mathbf{p}(t), \mathbf{q}(t))$, and a tunneling time is signaled by the stationarity with respect to time of $\bar{J}_{F}(t)$. At these times the trajectory is allowed to hop or tunnel with a probability given by Eq. (2.18) and the net tunneling probability $P_{\text {net }}\left(\mathbf{J}^{\prime} ; E, t\right)$, 


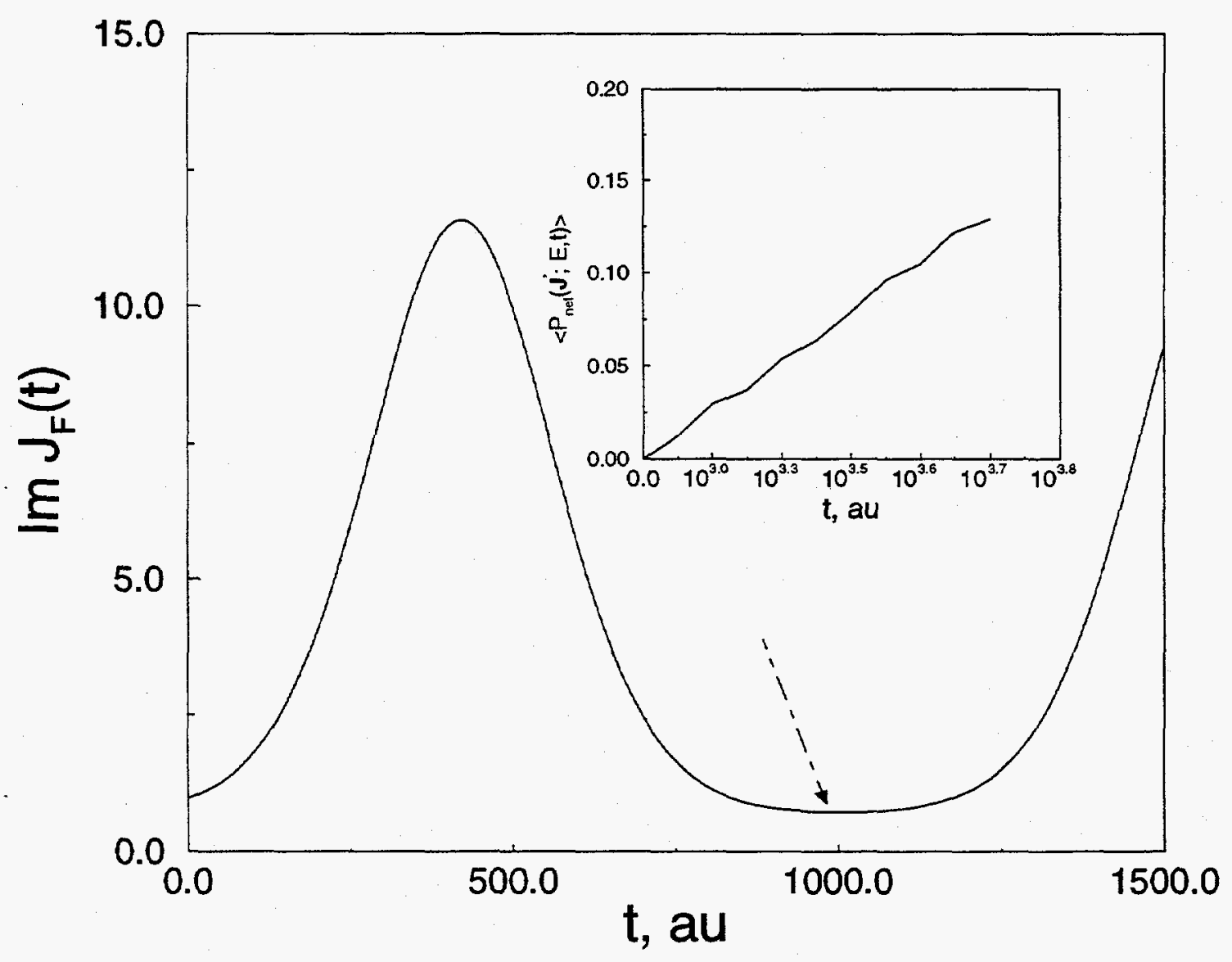

Figure 2.1: Shown here is a typical behaviour of $\bar{J}_{F}$ as a function of time and a tunneling time is indicated corresponding to the conserved value of $\bar{J}_{F}$. The inset shows a typical plot of the averaged net tunneling probability, $\left\langle P_{n e t}\left(\mathbf{J}^{\prime} ; E, t\right)\right\rangle$, as a function of time. 
neglecting back reaction,

$$
P_{n e t}\left(\mathbf{J}^{\prime} ; E, t\right)=\sum_{n} h\left(t-t_{n}\right) P\left(\mathbf{J}^{\prime} ; E\right)
$$

is calculated along the trajectory. Averaging $P_{\text {net }}\left(\mathbf{J}^{\prime} ; E, t\right)$ over appropriate inital conditions yields the average net tunneling probability, $\left\langle P_{\text {net }}\left(\mathbf{J}^{\prime} ; E, t\right)\right\rangle$. The rate constant for a unimolecular decay reaction is given by

$$
k_{u n i}=\frac{d}{d t}\left\langle P_{\text {net }}\left(\mathbf{J}^{\prime} ; E, t\right)\right\rangle \text {. }
$$

In the next section we apply this model to a two dimensional coupled unimolecular decay potential to demonstrate the accuracy of the model for predicting state-specific decay rates.

\section{3 $\quad k_{\text {uni }}$ for a model potential}

Our model potential is of the form

$$
V\left(q_{1}, q_{2}\right)=\frac{1}{2} a q_{1}^{2}-\frac{1}{3} b q_{1}^{3}+\frac{1}{2} m \omega^{2}\left(q_{2}-\frac{c q_{1}}{m \omega^{2}}\right)^{2}
$$

where the parameters $a$ and $b$ were chosen to correspond to a barrier height of $7.4 \mathrm{kcal} \mathrm{mol}^{-1}$, the mass $m$ taken to be that of a hydrogen atom, the harmonic frequency, $\omega$, was chosen to be $300 \mathrm{~cm}^{-1}$, and $c$ is the coupling constant. These values are typical of hydrogen atom transfer reactions. The perturbation calculation determining the locally conserved transition state actions as a function of cartesian variables was carried to second order using Lie transform perturbation theory ${ }^{3}$.

\subsubsection{Choice of initial conditions}

We want to calculate unimolecular decay rates from metastable (quasibound) initial states. We thus need to choose initial conditions for the classical trajectories that lie on a quantizing torus $\left(\mathcal{I}_{1}, \mathcal{I}_{2}\right)$, corresponding to our Hamiltonian,

\footnotetext{
${ }^{3}$ See appendix.
} 
and average over all initial angles $\left(\Phi_{1}, \Phi_{2}\right)$. In other words, a given specific metastable state corresponds semiclassically to a KAM torus ${ }^{4}[19]$.

A rigorous way of doing this is to use the method of adiabatic switching [29]. In this method, initial conditions are chosen from an appropriate KAM torus of some zeroth order Hamiltonian $H_{0}$, with the corresponding angles unformly distributed over the interval $[0,2 \pi)$. The remaining part of the Hamiltonian, $H-H_{0}$, is treated as a perturbation. Now the trajectories are allowed to evolve under the following time dependent Hamiltonian

$$
H(t)=H_{0}+\lambda(t)\left(H-H_{0}\right),
$$

where $\lambda(t)$, the switching function, starts out at zero and reaches unity at some later time $T$, the switching time. The principle of adiabatic invariance[30] guarantees that the actions remain approximately constant during the switching process whereas the energy changes to the correct value. At time $T$ we have the correct KAM torus from which the initial conditions for our calculations are chosen. Our choice for $H_{0}$ included the kinetic energy terms and an harmonic approximation for $V\left(q_{1}, q_{2}\right)$ about the minimum. The anharmonicities constitute the perturbation term which is switched on by using

$$
\lambda(t)=\frac{t}{T}-\frac{1}{2 \pi} \sin \left[\frac{2 \pi t}{T}\right] .
$$

At the end of the switching process the time $t$ is set equal to zero and the equations of motion are integrated with the full potential.

We have used another simpler procedure to choose initial conditions from the appropriate KAM torus. In this scaling method [31] initial conditions are chosen from an appropriate KAM torus corresponding to a zeroth order $H_{0}$, with the initial angles uniformly distributed over the interval $[0,2 \pi)$. We then rescale the harmonic initial momenta accordingly to achieve the necessary energy corresponding to the specific inital metastable state. This scaling procedure, though less rigorous as compared to the adiabatic switching method, is much more easily implemented in a truly large system. Adiabatic switching techniques are difficult to routinely apply to large systems.

\footnotetext{
${ }^{4}$ The assumption here is that the dynamics is regular.
} 
We performed the calculations described in this section with both methods and the differences were negligible.

\subsubsection{Results and Discussion}

The unimolecular decay rates for specific initial states were calculated using Eq. (2.23) where the averaging is done over the angle variables corresponding to the appropriate KAM torus. In order to compare our results to the exact quantum results, we computed the width of the metastable states using the method of complex scaling[32]. The $q_{1}$ coordinate was rotated as $q_{1} \rightarrow q_{1} e^{i \alpha}$, while the $q_{2}$ coordinate remained real. The resulting complex scaled Hamiltonian was diagonalized in a real basis set and the complex eigenvalues

$$
\mathcal{E}=\mathcal{E}_{\tau}-\frac{i}{2} \mathcal{E}_{i},
$$

which were stable under change of the scaling angle $\alpha$ were identified as resonances. The decay rate is then given by

$$
k_{u n i}=\frac{\mathcal{E}_{i}}{\hbar}
$$

Shown in figures $2.2,2.3,2.4$ are the semiclassical $k_{u n i}$ along with the accurate quantum results for various initial states as a function of the energy of the state. For the specific parameters chosen for our model potential there are about seven bound metastable states. As the perturbation calculations were done about the saddle point the model is not expected to perform as well for states lying far below the barrier. However, as a test we calculated the rates for all the metastable states. In the figures $\left(n_{1}, n_{2}\right)$ denotes the specific initial reactant state for which the calculations were done where $n_{1}$ corresponds to the reactive, $q_{1}$, mode. Each point was averaged over 1000 trajectories, but the results are reasonably converged with as few as a couple of hundred trajectories.

In the case of figure (2.2) with zero coupling constant the semiclassical results compare very well with the exact quantum results. This is to be expected since for $c=0$ the system is integrable and effectively an one dimensional problem. The perturbation calculation combined with the uniformly valid tunneling probability 


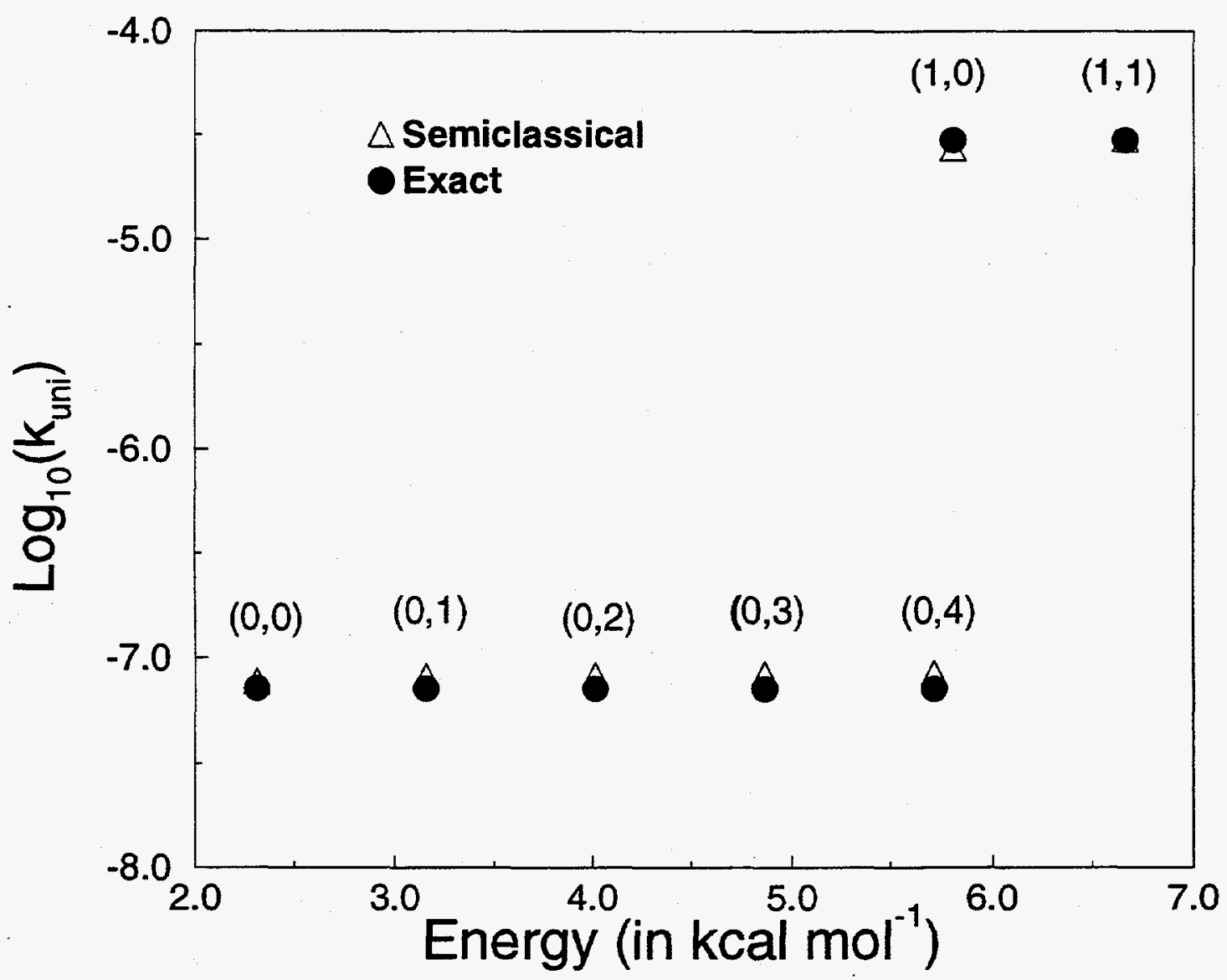

Figure 2.2: Unimolecular deacy rates for specific reactant quantum states $\left(n_{1}, n_{2}\right)$ as a function of the energy of the state. The coupling constant $c$ is equal to zero. Each point is averaged over 1000 trajectories. Notice the strong mode specificity between the states $(0,4)$ and $(1,0)$. 


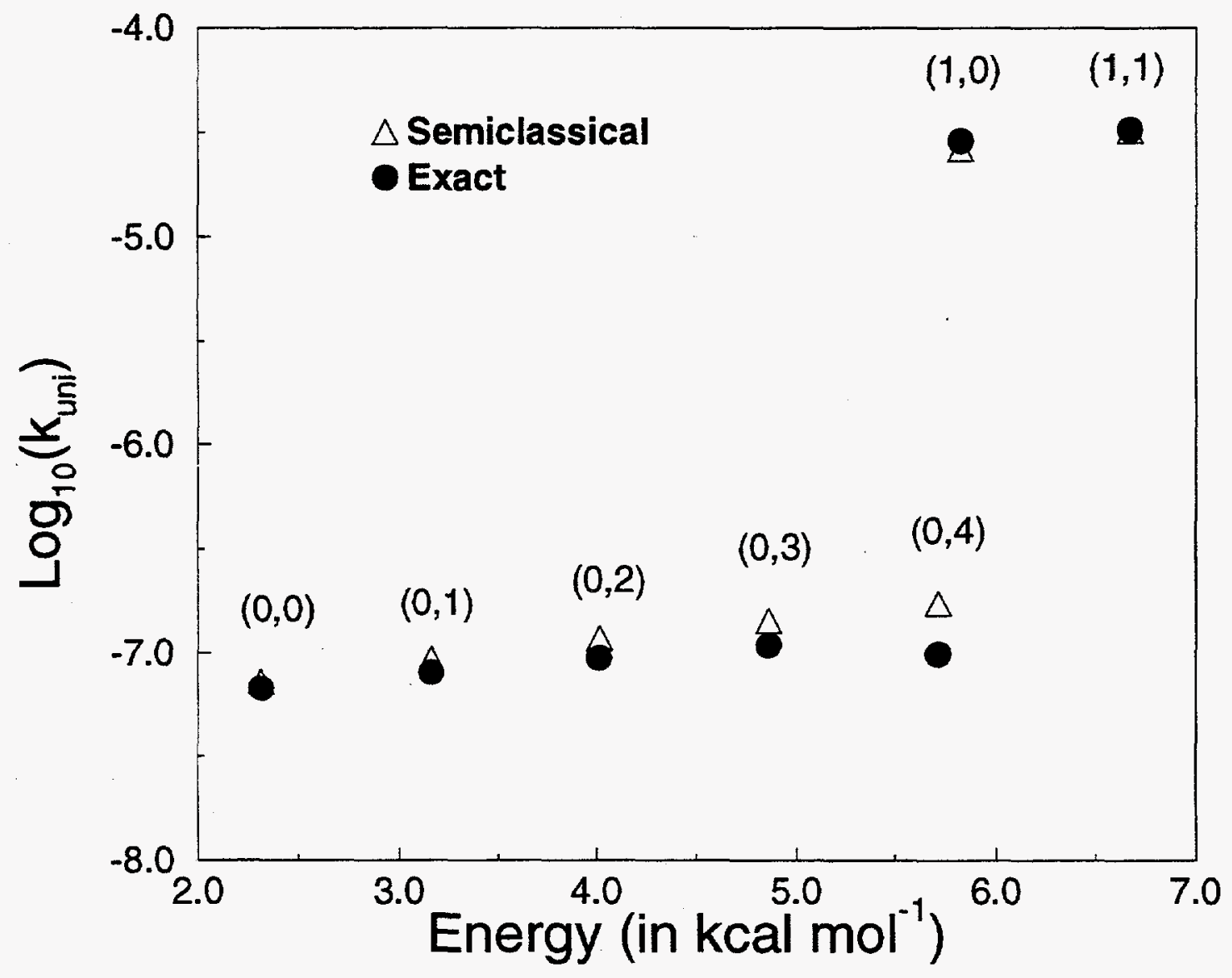

Figure 2.3: Unimolecular decay rates for coupling constant $c=0.001 \mathrm{au}$. Each point is averaged over 1000 trajectories. Strong mode specificity still persists at this value of $c$. 


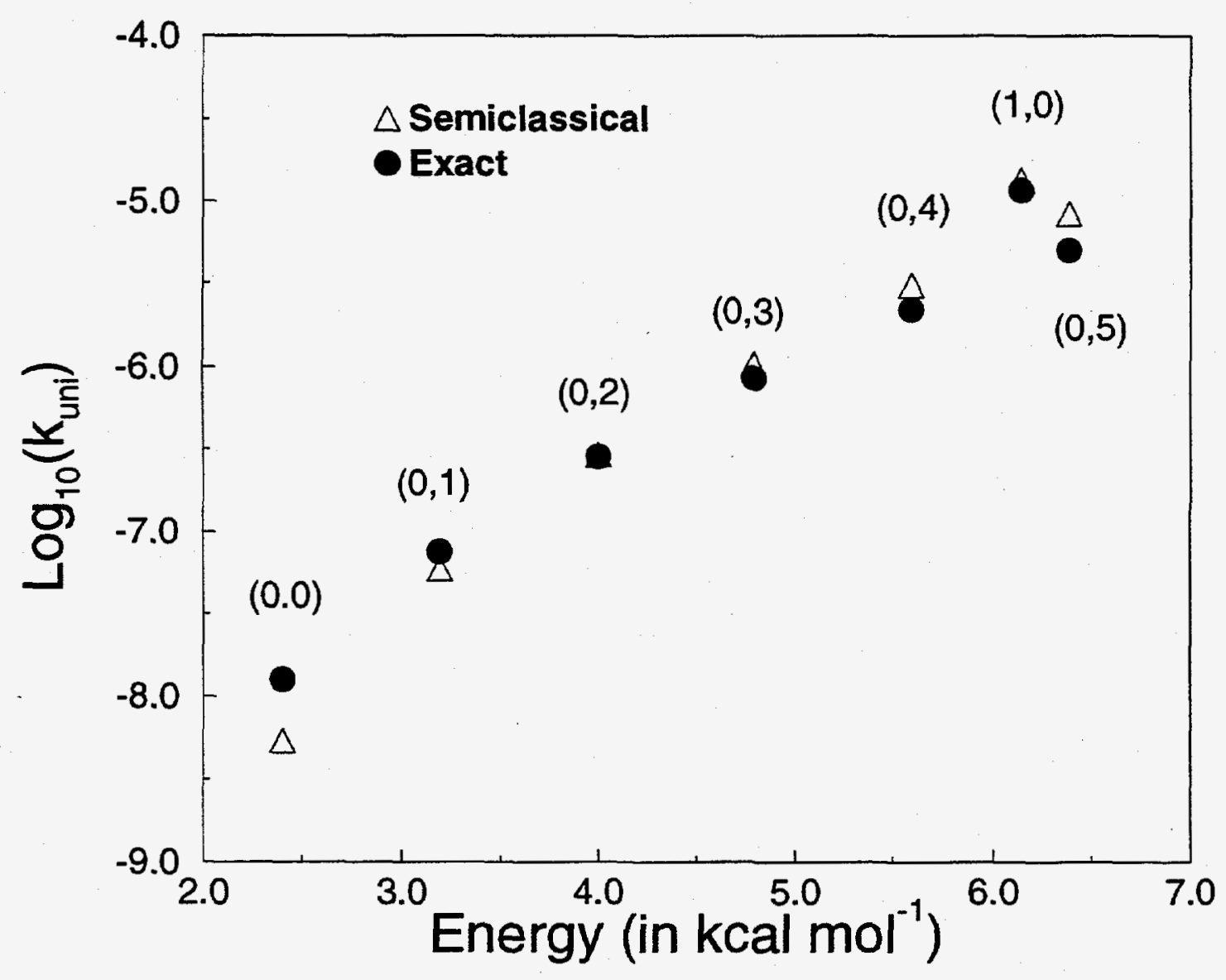

Figure 2.4: Unimolecular decay rates for coupling constant $c=0.005$ au. Each point is averaged over 1000 trajectories. The mode specificity has almost vanished and the rates are purely a function of the total energy. Also note the nontrivial dependence of rate at high energies i.e., $(0,5)$ state has a lower rate than the $(1,0)$ state. This feature is also predicted by the semiclassical model. 
of Eq. (2.18) is as good as the WKB result. For the case of moderate coupling, $c=0.001 \mathrm{au}$, in figure (2.3) the semiclassical model again performs well for all low lying states. For both $c=0 a u$ and $c=0.001 a u$ we see a strong mode specificity in the decay rates. As an example, for the moderate coupling case the states $(0,4)$ and $(1,0)$ differ in energy by less than $0.1 \mathrm{kcal} \mathrm{mol}^{-1}$ but the corresponding rates differ by almost two orders of magnitude. Our model is successful in describing this mode specific effect. There is a lot of interest in mode specific chemistry[36] and it is significant that our simple model can account for this effect. Figure (2.4) shows the results for the highest coupling constant, $c=0.005 \mathrm{au}$. There is no mode specificity and the rates are almost a function only of the total energy of the system. The semiclassical rates agree very well with the exact rates for states close to the barrier. For states far below the barrier the agreement with exact values of the rate are not as good since the second order perturbation results at this high coupling are not as reliable. Note the slight decrease in the decay rate of the state $(0,5)$ relative to the state $(1,0)$. This nontrivial feature is also successfully captured by the semiclassical model. It is impressive to note that the semiclassical results in the worst case are off from the exact quantum rates by a factor of two.

\subsection{Concluding remarks}

In this chapter we have outlined a semiclassical model for multidimensional tunneling which is based on locally conserved actions. The model performs very well in predicting unimolecular decay rates from specific initial reactant states. More significantly, it is dynamically self-consistent and requires fewer ad hoc assumptions in order to define it as compared to earlier models for tunneling. As the tunneling actions and times are determined in a dynamically consistent fashion, this model has the ability to directly incorporate the underlying, presumably complicated, dynamics of the system of interest. In the present version it is possible to exploit the information around stationary states on the potential surface provided by $a b$ initio quantum chemistry calculations. This model certainly seems to show promises of applicability to real chemical systems where the tunneling motion of hydrogen atoms is an 
important aspect of the reaction dynamics.

\subsubsection{Comments on the choice of $t_{n}$}

It is satisfying to note that the semiclassical model described above is capable of obtaining reliable tunneling decay rates for specific reactant states. However we have not addressed the question as to why does this perturbative model give such encouraging results even though the dynamics is nonintegrable. In this section we will attempt to answer the question which, in turn possibly leads to a clearer mathematical and physical basis for our choice of the tunneling action and times.

Realizing the hopelesness of finding a canonical transformation, or a series of canonical transformations which would reduce our Hamiltonian to a function of actions alone, we put forward a perturbative model. In order to be of practical utility the perturbation series is truncated at a certain order. Note that the formal perturbation series almose always diverges. The resulting truncated Hamiltonian allows us to study the behaviour of the system of interest over an appreciable period of time for inital conditions close to the point in phase space $z_{0}$ about which the perturbation is done[19]. In other words, by truncating the perturbation series we have replaced our original nonintegrable Hamiltonian with an integrable one ${ }^{5}$. This makes sense as locally every Hamiltonian is integrable ${ }^{6}$. However, it would be erroneous to calculate observables corresponding to our original Hamiltonian by doing dynamics on the truncated, integrable Hamiltonian. This is because trajectories which lead to important contribution could have initial conditions which are nowhere close to the region around which the perturbation analysis was performed. This error would be especially serious for tunneling which is inherently an observable corresponding to long time dynamics. Thus, the classical dynamics must correspond to the original Hamiltonian and when the exact trajectory is in the neighbourhood of $z_{0}$ the corresponding actions and energies are approximated well by the perturbation analysis. In fact in the time interval when the imaginary action is approximately conserved, the

\footnotetext{
${ }^{5}$ We are assuming for the argument that there are no resonances. In presence of resonances the argument can be suitably modified.

${ }^{6}$ This is the celebrated Darboux's theorem in classical mechanics[19].
} 


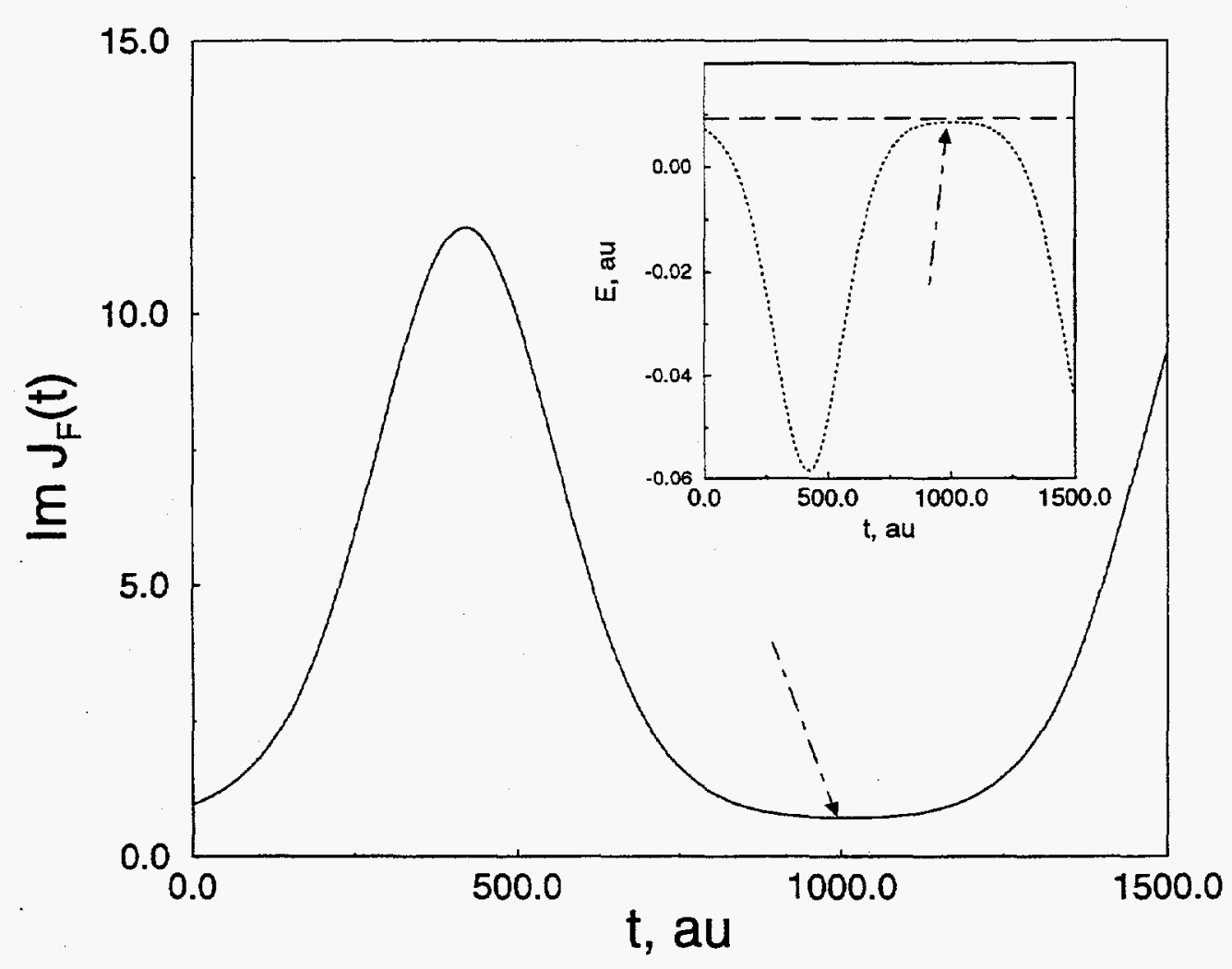

Figure 2.5: In the main figure $\bar{J}_{F}(t)$ is shown as a function of time. The inset shows the exact trajectory energy $E$ (dashed line) and the perturbative energy $E_{\text {pert }}$ (dotted line) as a function of time along the same trajectory. Notice that $E_{p e r t}$ and $\bar{J}_{F}(t)$ are locally conserved in the same time interval. In that interval the system is locally integrable.

value of the truncated Hamiltonian is very close to the exact energy of the trajectory. In that particular time interval it is plausible that there exist $F$ locally conserved quantities which render the dynamics locally integrable. For example, as shown in figure 2.5, in our two dimensional model case presented earlier the two conserved quantities happen to be $\bar{J}_{F}$ and the value of the truncated Hamiltonian $E_{\text {pert }}$. The real action is not conserved except at very low coupling constants.

At this stage we will briefly consider the rigorous approach to tunneling based on analytically continued classical mechanics[11]. In a conservative one dimensional system, which is always integrable, it is relatively simple to calculate tunneling actions by considering complex time trajectories. In fact one can choose a proper 
contour in complex time plane to keep the coordinate real. However, for a general multidimensional nonintegrable system it is not possible to keep all of the coordinates real but one. Thus there is no way to avoid dealing with complex valued trajectories. Suppose the multidimensional $F$ degree of freedom system is indeed integrable and all of the constants of motion are known. In this case it is still possible to keep all of the coordinates real because one has $F$ independent time like parameters which can be chosen appropriately to keep the trajectory real ${ }^{7}$. What happens in the situation when we do not have global contants of motion but only local constants of motion? This is exactly where the connection lies to our semiclassical model described in this chapter. In our model we do have, over a certain time interval, a locally integrable system and we know the corresponding locally conserved quantities. We then use the values of various dynamical quantities along the numerically exact trajectory in that time interval to determine, perturbatively, the tunneling action. Our model has implicitly chosen the right branches and the correct tunneling action to that order in perturbation theory. This is perhaps some justification for our choice of the tunneling time and the action.

\subsection{Appendix I: Classical perturbation theory}

Classical perturbation theory has a long illustrious history going back to Poincaré and Von Zeipel. Reports on progress in perturbation theory applied to systems where they fail and do not fail can be found in the articles by Chirikov[33] and Cary[27]. In this appendix we will give a brief introduction to Lie transform perturbation theory ${ }^{8}$ which is a more efficient method utilizing the natural structure assosciated with infinitesimal canonical transformations. In particular, the detailed differences between previous methods and Lie transform methods will not be presented.

\footnotetext{
${ }^{7}$ For example, these could be the angle variables on the KAM torus in phase space. Remember, for a harmonic oscillator $\psi(t)=\psi(0)+\omega t$.

${ }^{8}$ Most of this appendix relies heavily on the unpublished notes of Prof. Littlejohn.
} 


\subsubsection{Notations}

In order to keep the presentation clear we will adopt the following notations. Any point in phase space is denoted by $\mathbf{z} \equiv(\mathbf{p}, \mathbf{q})$. We introduce a $2 F \times 2 F$ antisymmetric matrix $\Gamma$ given by its partition into four $F \times F$ matrices

$$
\Gamma=\left(\begin{array}{cc}
\mathbf{0} & \mathbf{I} \\
-\mathbf{I} & \mathbf{0}
\end{array}\right)
$$

With this notation we can write down Hamilton's equation of motion as

$$
\frac{d \mathbf{z}}{d t}=\boldsymbol{\Gamma} \cdot \frac{\partial H}{\partial \mathbf{z}} .
$$

We will also use a notation for Poisson brackets given by

$$
\begin{aligned}
L_{A} B & \equiv\{A, B\}=\frac{\widetilde{\partial A}}{\partial \mathbf{z}} \cdot \boldsymbol{\Gamma} \cdot \frac{\partial B}{\partial \mathbf{z}} \\
& =\sum_{k, l=1}^{2 F} \frac{\partial A}{\partial z_{k}} \Gamma_{k l} \frac{\partial B}{\partial z_{l}} \equiv \sum_{k, l=1}^{2 F} A_{, k} \Gamma_{k l} B_{, l}
\end{aligned}
$$

where $A$ and $B$ are some functions on phase space. Notice the notation we are using for derivatives of functions on phase space with respect to the coordinates. In particular, the Poisson brackets of the coordinates among themselves are just the components of the matrix $\Gamma$,

$$
\left\{z_{k}, z_{l}\right\}=\Gamma_{k l} .
$$

\subsubsection{Transformation operators}

In this section we will obtain an operator representation of a canonical transformation. We will assosciate a canonical transformation $\mathbf{z}^{\prime}=\mathbf{Z}(\mathbf{z})$ with an operator $T$, defined by

$$
(T f)(\mathbf{z})=f(\mathbf{Z}(\mathbf{z}))
$$

which is written as

$$
T f=f \circ \mathbf{Z},
$$


where $o$ is the operation of composition. $T$ is an operator which maps old functions into new functions, in terms of which we can write the transformation of Hamiltonians in the form,

$$
H(\mathbf{z})=K\left(\mathbf{z}^{\prime}\right)=(K \circ \mathbf{Z})(\mathbf{z})
$$

or,

$$
H=T K
$$

Note that $T$ transforms the new Hamiltonian $K$ into the old one $H$ and not the other way. The inverse transformation of $\mathbf{z}^{\prime}=\mathbf{Z}(\mathbf{z})$ is written down as

$$
\mathbf{z}=\mathbf{Y}\left(\mathbf{z}^{\prime}\right)
$$

It is easy to check that

$$
\mathbf{Y} \circ \mathbf{Z}=\mathbf{Z} \circ \mathbf{Y}=\mathbf{I}
$$

where $\mathbf{I}$ is the identity function i.e., $I_{k}(\mathbf{z})=z_{k}$ for all $\mathbf{z}$.

Using $\mathbf{Y}$ we can define the inverse transformation operator as

$$
\left(T^{-1} f\right)(\mathbf{z})=f(\mathbf{Y}(\mathbf{z}))
$$

If we now compute the action of $T T^{-1}$ on a function $f$ by evaluating at a point $\mathbf{z}$ then we get the function back. Hence, $T T^{-1}=\mathcal{I}$ is the identity transformation.

Now let us consider a canonical transformation $\mathbf{Z}(\mathbf{z}, \epsilon)$ which is a solution of the following Hamilton's equation

$$
\frac{\partial Z_{k}(\mathbf{z}, \epsilon)}{\partial \epsilon}=\Gamma_{k l} w_{, l}(\mathbf{Z}(\mathbf{z}, \epsilon))
$$

where $w$ is the "Hamiltonian" and $\epsilon$ is the variable of evolution. The function $w$ is not the real Hamiltonian, but a function used in Hamilton's equations to generate a canonical transformation. This is in direct analogy with the usual situation when the real Hamiltonian is used to generate time evolution which is a canonical transformation[19]. Just as the Hamiltonian is called as the generator of time evolution, we will call $w$ as the Lie generator of the canonical transformation $\mathbf{z}=\mathbf{Z}(\mathbf{z}, \epsilon)$. 
The initial conditions for Eq. $(2.40)$ is $\mathbf{Z}(\mathbf{z}, 0)=\mathbf{z}$ for all $\mathbf{z}$. Given this $\epsilon$ dependent canonical transformation, we associate an $\epsilon$ dependent operator,

$$
(T(\epsilon) f)(\mathbf{z})=f(\mathbf{Z}(\mathbf{z}, \epsilon))
$$

Differentiating both sides of Eq. (2.41) with respect to $\epsilon$ we have

$$
\begin{aligned}
\left(\frac{d T(\epsilon)}{d \epsilon} f\right)(\mathbf{z}) & =f_{, l}(\mathbf{Z}(\mathbf{z}, \epsilon)) \frac{\partial Z_{l}(\mathbf{z}, \epsilon)}{\partial \epsilon} \\
& =f_{l l}(\mathbf{Z}(\mathbf{z}, \epsilon)) \Gamma_{l k} w_{, k}(\mathbf{Z}(\mathbf{z}, \epsilon))=\{f, w\}(\mathbf{Z}(\mathbf{z}, \epsilon)) \\
& =(T(\epsilon)\{f, w\})(\mathbf{z})=-\left(T(\epsilon) L_{w} f\right)(\mathbf{z}) .
\end{aligned}
$$

This holds for all $\mathbf{z}$ and $f$ and hence we have

$$
\frac{d T(\epsilon)}{d \epsilon}=-T(\epsilon) L_{w}
$$

which is an operator equation. Assuming that $L_{w}$ is independent of $\epsilon$, i.e., $w$ has no explicit dependence on $\epsilon$ we can integrate Eq. (2.43) easily to obtain

$$
T(\epsilon)=e^{-\epsilon L_{w}}
$$

A canonical transformation expressed in this form is called a Lie transform. Equation (2.44) is an operator representation of a canonical transformation.

\subsubsection{Perturbation series}

To get explicit formulae connecting old and new variables we use the fact that

$$
(T(\epsilon) \mathbf{I})(\mathbf{z})=\mathbf{Z}(\mathbf{z}, \epsilon),
$$

and use Eq. (2.44) by expanding the exponential to obtain

$$
\mathbf{Z}(\mathbf{z}, \epsilon)=\mathbf{z}-\epsilon\{w, \mathbf{z}\}+\frac{\epsilon^{2}}{2}\{w,\{w, \mathbf{z}\}\}-\ldots
$$

Similar equation is obtained for the inverse transformation. The transformed Hamiltonian $K=T^{-1} H$ is obtained to required order in $\epsilon$ by expanding both $H$ and $K$ in powers of $\epsilon$

$$
\begin{aligned}
\left(K_{0}+\epsilon K_{1}+\epsilon^{2} K_{2}+\ldots\right)= & \left(\mathcal{I}+\epsilon L_{w}+\frac{\epsilon^{2}}{2} L_{w}^{2}+\ldots\right) \\
& \times\left(H_{0}+\epsilon H_{1}+\epsilon^{2} H_{2}+\ldots\right) .
\end{aligned}
$$


Equating powers of $\epsilon$ on both sides of Eq. (2.47) we get

$$
\begin{array}{ll}
O\left(\epsilon^{0}\right): & K_{0}=H_{0} \\
O\left(\epsilon^{1}\right): & K_{1}=H_{1}+L_{w} H_{0} \\
O\left(\epsilon^{2}\right): & K_{2}=H_{2}+L_{w} H_{1}+\frac{1}{2} L_{w}^{2} H_{0} .
\end{array}
$$

For example, $H_{0}$ would correspond to a solvable Hamiltonian.

Suppose now that our original Hamiltonian $H$ was expresses in terms of the action-angle variables. These action-angle variables correspond to the solvable $H_{0}$. The aim here is to choose the generating function $w$ such that our new Hamiltonian at $O(\epsilon)$ is independent of the angle variables and $w$ itself is independent of secular terms in the angles. Secular terms are terms which are unbounded in time. For example, $\psi$ is a secular term and $\sin (\psi)$ is not secular. Thus the presence of secular terms causes the perturbation solution to diverge from the true solution at long times. In order to satisfy these requirements, say at $O\left(\epsilon^{1}\right)$, the following choice of $K_{1}$ and $w$ is made

$$
\begin{aligned}
K_{1} & =\bar{H}_{1} \\
L_{w} H_{0} & =-\widetilde{H}_{1},
\end{aligned}
$$

where $\bar{H}_{1}$ and $\widetilde{H}_{1}$ denote the average and oscillatory parts of $H_{1}$ respectively. They are defined as

$$
\begin{aligned}
\bar{H}_{1}(\mathbf{J}) & =\frac{1}{(2 \pi)^{F}} \int_{0}^{2 \pi} d \boldsymbol{\psi} H_{1}(\mathbf{J}, \boldsymbol{\psi}) \\
\widetilde{H}_{\mathbf{1}}(\mathbf{J}, \boldsymbol{\psi}) & \equiv H_{1}(\mathbf{J}, \boldsymbol{\psi})-\bar{H}_{1}(\mathbf{J})
\end{aligned}
$$

A more efficient way is to slightly generalize our transformation operator to

$$
T(\epsilon)=e^{-\epsilon L_{1}} e^{-\epsilon^{2} L_{2}} e^{-\epsilon^{3} L_{3}} \ldots,
$$

where we are now doing a sequence of transformations and each one comes with its own Lie generator $w_{j} \leftrightarrow L_{j}$. We can write down the new Hamiltonian as

$$
K=\ldots . . e^{\epsilon^{2} L_{2}} e^{\epsilon L_{1}} H .
$$


This version of Lie transform perturbation theory is very similar to canonical VanVleck perturbation theory[28] for the quantum analog of our classical Hamiltonian. In particular, if we make the formal Dirac quantization[34] of the Poisson bracket

$$
L_{w} \rightarrow \frac{1}{i \hbar} \mathcal{L}_{w},
$$

where

$$
\mathcal{L}_{w} \widehat{A} \equiv[\widehat{w}, \widehat{A}],
$$

is the usual quantum commutator, then we can write the quantum analog of Eq. (2.52) as

$$
\widehat{K}=\ldots . e^{\epsilon^{2} \mathcal{L}_{2}} e^{\epsilon \mathcal{L}_{1}} \widehat{H} .
$$

Continuing the analysis along similar lines as described above we can get the new Hamiltonian to the desired order in $\epsilon$. Again, at each order the generating function is chosen such that the new Hamiltonian at that order does not depend on the angles and there are no secular terms in the generating function.

\subsubsection{Simple example}

In this section we will apply Lie transfrom perturbation theory to a simple one dimensional example. The Hamiltonian is

$$
H(p, q)=\frac{1}{2} p^{2}+\frac{1}{2} q^{2}-\frac{1}{3} q^{3} .
$$

This is just the uncoupled case of our two dimensional model Hamiltonian considered in this chapter. For clarity the parameters have been scaled to unity. The maximum for this potential is at $q^{\dagger}=1$. Around this maximum we can write our Hamiltonian as

$$
\begin{aligned}
h(q, p) & \equiv H(q, p)-\frac{1}{6} \\
& =\frac{1}{2} p^{2}+\frac{1}{2}\left(q-q^{\dagger}\right)^{2}-\frac{1}{3}\left(q-q^{\dagger}\right)^{3} .
\end{aligned}
$$

Now we make the following action-angle transformations

$$
\begin{aligned}
& p=\sqrt{2 J} \cos \psi \\
& q=\sqrt{2 J} \sin \psi+q^{\dagger} .
\end{aligned}
$$


Hence we can formally write our Hamiltonian in terms of these action-angle variables as

$$
h(J, \psi)=J+\epsilon h_{1},
$$

where $\epsilon$ is a formal perturbation parameter and

$$
h_{1}(J, \psi) \equiv-\frac{1}{3}(2 J)^{\frac{3}{2}} \sin ^{3} \psi .
$$

It is clear that at $O\left(\epsilon^{0}\right)$

$$
K_{0}=H_{0}=J^{\prime} .
$$

$J^{\prime}$ is the corrected action. To zeroth order it can be replaced by $J$. Similarly for the corrected angle $\psi^{\prime}$. $\left(J^{\prime}, \psi^{\prime}\right)$ can be obtained from Eq. (2.46). At $O\left(\epsilon^{1}\right)$ we obtain

$$
\begin{aligned}
& K_{1}=\bar{h}_{1}=0 \\
& w_{1}=\frac{1}{36}\left(2 J^{\prime}\right)^{\frac{3}{2}}\left(\cos 3 \psi^{\prime}-9 \cos \psi^{\prime}\right) .
\end{aligned}
$$

Hence we have to go to $O\left(\epsilon^{2}\right)$ in order to get the first correction to our Hamiltonian. At this order the relevant equation is

$$
2 L_{2} h_{0}=2\left(K_{2}-h_{2}\right)-L_{1}\left(h_{1}+K_{1}\right) .
$$

In our example, $h_{n} \equiv 0$ for all $n>1$ and $K_{1}=0$. We get the following results at $O\left(\epsilon^{2}\right)$

$$
\begin{aligned}
& K_{2}=\frac{1}{2} \overline{\left\{w_{1}, h_{1}\right\}}=-\frac{5}{12} J^{\prime 2} \\
& w_{2}=-\frac{1}{48} J^{\prime 2}\left(8 \sin 2 \psi^{\prime}+\sin 4 \psi^{\prime}\right) .
\end{aligned}
$$

We can easily continue this analysis and at $O\left(\epsilon^{4}\right)$ obtain the new Hamiltonian as

$$
K=J^{\prime}-\frac{5}{12} J^{\prime 2}-\frac{235}{432} J^{\prime 3},
$$

where we have set the formal perturbation parameter $\epsilon=1$. Now the action $J^{\prime}$ is pure imaginary as we are doing perturbation around the barrier. By substituting

$$
J^{\prime}=\frac{i \hbar}{\pi} \theta
$$


and inverting Eq. (2.65) we obtain the barrier penetration integral $\theta$ for our example problem as

$$
\theta \approx \frac{\pi}{\hbar}\left[-K+\frac{5}{12} K^{2}-\frac{385}{432} K^{3}\right] .
$$

By identifying $K=\left(E-\frac{1}{6}\right)$ we can prove that the resulting $\theta$ is the same as that obtained from approximations on the usual definition of the one dimensional barrier penetration integral[35]. 


\section{Chapter 3}

\section{Semiclassical Scattering Theory}

\subsection{Introduction}

Chemical reactions at the most fundamental level are scattering processes i.e., collision between two chemical species leading to products. For example, the bimolecular reaction

$$
\mathrm{H}+\mathrm{H}_{2} \mathrm{O} \rightarrow \mathrm{H}_{2}+\mathrm{OH}
$$

involves a hydrogen atom colliding with a water molecule to give hydrogen molecule and hydroxy radical as products. As chemists we are interested in obtaining the rate of a chemical reaction and the effect of various vibrational-rotational couplings of the reactants on the rate. The advent of modern experimental techniques which include molecular beam methods[38] and high resolution spectroscopies[39] has made it possible to measure the rates of chemical reactions in a very detailed fashion. These experimental advances have provided a great impetus for theoreticians to calculate rates of chemical reactions from the fundamental viewpoint of scattering theory.

The most detailed characterization of a bimolecular reaction is obtained by computing the scattering matrix (S-matrix) for the chemical reaction. The S-matrix, $S_{\mathbf{n}_{p}, \mathbf{n}_{r}}(E)$, gives the amplitude for reaction at a total energy $E$. The quantum numbers $\mathbf{n}_{p}$ and $\mathbf{n}_{r}$ characterize the internal asymptotic states of the products and reactants respectively. For instance, in Eq. (3.1), $\mathbf{n}_{r}$ describes the initial vibrational states of the water molecule. Once the S-matrix for a reaction has been calculated, the thermal 
rate constant is given by[40]

$$
k(T)=\frac{1}{2 \pi \hbar Q_{r}(T)} \int_{-\infty}^{\infty} d E e^{-\beta E} N(E)
$$

where $\beta=\left(k_{B} T\right)^{-1}, k_{B}$ is the Boltzmann's constant, and $Q_{r}(T)$ is the reactant partition function including relative translational motion, per unit volume. The microcanonical cummulative reaction probability $N(E)$ is defined as[40]

$$
N(E)=\sum_{\mathbf{n}_{p}} \sum_{\mathbf{n}_{r}}\left|S_{\mathbf{n}_{p}, \mathbf{n}_{r}}(E)\right|^{2}
$$

In recent years, considerable amount of progress has been made in the methodologies for calculating the S-matrix via exact quantum reactive scattering (QRS) theory[41]. The S-matrix Kohn variational principle (KVP) formulation of QRS[42] represents an important milestone as far as exact quantum approaches to reactive scattering are concerned. However, the computational effort required in these exact approaches in order to calculate the entire S-matrix is considerable. This numerical bottleneck, despite ingenious numerical strategies, has prevented applications to more than three atom systems. On the other hand, the fact that $k(T)$ and $N(E)$ are average quantities leads one to believe that it must be possible to calculate them directly without obtaining the full S-matrix for the system of interest. This important observation has led to dramatic advances in the theory of reaction dynamics for calculating the rates for much larger systems. Miller, Schwartz and Tromp proposed a method based on flux-flux correlation functions[43] in order to directly calculate $k(T)$. More recently, a whole range of direct methods[44] have been put forward to directly compute $N(E)$. In all of the direct methods to calculate $N(E)$ the central object of interest is the outgoing wave energy Green's function $G^{+}(E)$. The aim is to obtain a convienient, well-behaved representation of $G^{+}(E)$ which has numerically attractive features. In the recent methods this is achieved by using a discrete variable representation [45] for the Hamiltonian with absorbing boundary conditions (DVRABC) $[44,46]$. This makes the resulting Hamiltonian matrix sparse which can now be dealt with by using iterative techniques[48]. Investigations in these directions have led to a very promising approach for calculating $k(T)$ for large systems[49]. 
It is important to realize that the significant advances in rigorous quantum mechanical calculations are not only due to these new developements in theoretical methodologies but also due to continued increase in computational power. It is now possible to calculate $k(T)$ and $N(E)$ for four atom systems. In fact the most complex reaction that has been described rigorously (i.e. in its full dimensionality, six degrees of freedom) is $\mathrm{H}_{2}+\mathrm{OH} \rightarrow \mathrm{H}+\mathrm{H}_{2} \mathrm{O}$ [50]. Even for these direct calculations, going beyond four atom systems warrants new numerical techniques and better computing machines. It is important to note that in order to perform reaction dynamics, classical or quantum, we require global $a b$ initio potential energy surfaces which, with current $a b$ initio technologies, are limited to a few atom systems. Nevertheless, from a purely numerical standpoint the memory requirements for an exact calculation on a system with greater than four atoms is very large. However, it is well known that performing classical trajectory calculations on large systems is not memory intensive. This has led to a considerable amount of work in the area of quasiclassical trajectories based scattering calculations[51]. In most cases quasiclassical trajectory calculations have preceded exact calculations in order to obtain rates and cross-sections for reactions[51, 52]. These calculations, despite their importance as far as to provide a qualitative and semi-quantitative description of the reaction, have some significant drawbacks. One of the drawbacks is that they cannot account for classically forbidden processes. This implies that close to the reactive threshold the calculations are not very reliable. The other significant, long standing problem in classical trajectory calculations is the zero point energy problem[53]. In quasiclassical trajectory calculations this is taken care of by rejecting those trajectories which lead to products with the wrong zero point energies. This is the best that can be done as to date there is no consistent and practical way of including zero point energy effects in quasiclassical trajectory simulations[54].

One way to overcome the drawbacks mentioned above is to use semiclassical methods. Semiclassical dynamics can account for classically forbidden processes and incorporate the correct zero point energies. At the same time the rest of the attractive features of classical trajectory simulations are kept intact. Of course there is a price to be paid i.e., now the amplitudes and phases assosciated with each classical trajectory 
have to be calculated. There already exist semiclassical theories which have had considerable success in calculating reaction rates. Of these theories, classical $S$-matrix theory[9] developed by Miller has a special place in semiclassical dynamics due both to its elegant formulation and applicability. In traditional S-matrix theory classically forbidden processes are handled by complex valued trajectories[11]. This feature is perhaps the only one that limits its applicability to large systems wherein tunneling plays a significant role. However, as it will be demonstrated in this chapter, it is possible to alleviate the problem if the stationary phase approximation is not imposed on the classical S-matrix theory. For a complex system consisiting of many strongly coupled degrees of freedom it is plausible that an entirely semiclassical theory would not perform very well. An attractive possibility is to come up with a hybrid method which would combine an accurate quantum treatment of the few strongly coupled degrees of freedom with a semiclassical treatment of the rest. In this fashion we would be using the information from a reduced dimensionality quantum calculation and semiclassically correcting for the weakly coupled degrees of freedom that were ignored in the quantum calculation. In addition, if the weakly coupled modes are approximately harmonic then semiclassical approaches are essentially exact. Feynman path integral methods[5] offer one approach for devising methods of this type, as does the time-dependent self-consistent field (TDSCF) method[6, 56].

In this chapter another approach for combining an approximate quantum description with a semiclassical approximation is described[55]. This approach is based on time-independent scattering theory. The motivation for this method is due to the recent work of Heller and coworkers[58]. They showed that primitive semiclassical approximations can be surprisingly accurate, even for long times and in situations where the classical dynamics is chaotic. The results for a nontrivial test problem indicate that it is indeed possible to accurately correct an approximate quantum calculation within this semiclassical approach. Interestingly enough, contributions due to tunneling are accounted for rather well with purely real time classical trajectories.

A brief description of S-matrix KVP is given in the next section. The semiclassical hybrid approach and results are discussed in the following sections. Finally, conclusions and important observations are discussed. 


\subsection{S-Matrix Kohn variational principle}

In this section we introduce the S-matrix version of the Kohn variational principle for reactive scattering[61]. In order to be general we will explicitly denote the various chemical arangements (channels) by $\alpha$. For a given arrangement we denote the radial scattering coordinate by $R_{\alpha}$ and the internal coordinates by $\mathbf{r}_{\alpha}$. A formally exact expression for the S-matrix elements in the distorted Born representation is

$$
\begin{aligned}
S_{\mathbf{n}^{\prime} \alpha^{\prime}, \mathbf{n} \alpha}(E) & =S_{\mathbf{n}^{\prime} \alpha^{\prime}, \mathbf{n} \alpha}^{0}(E) \\
& +\frac{i}{\hbar}\left\langle\Phi_{\mathbf{n}^{\prime} \alpha^{\prime}}|\hat{H}-E| \Phi_{\mathbf{n} \alpha}\right\rangle \\
& +\frac{i}{\hbar}\left\langle\varphi_{\mathbf{n}^{\prime} \alpha^{\prime}}\left|\hat{G}^{+}(E)\right| \varphi_{\mathbf{n} \alpha}\right\rangle
\end{aligned}
$$

where $\hat{G}^{+}(E) \equiv \lim _{\epsilon \rightarrow 0^{+}}(E+i \epsilon-\hat{H})^{-1}$ is the scattering Green's function with outgoing wave boundary conditions and

$$
\begin{aligned}
\varphi_{\mathbf{n} \alpha} & \equiv(\hat{H}-E) \Phi_{\mathbf{n} \alpha} \\
\varphi_{\mathbf{n}^{\prime} \alpha^{\prime}} & \equiv(\hat{H}-E) \Phi_{\mathbf{n}^{\prime} \alpha^{\prime}}
\end{aligned}
$$

In Eq. (3.4), $\mathbf{n} \alpha$ and $\mathbf{n}^{\prime} \alpha^{\prime}$ denote reactant and product channels respectively and $S_{\mathbf{n}^{\prime} \alpha^{\prime}, \mathbf{n} \alpha}^{0}(E)$ is the unitary reference S-matrix assosciated with the trial wavefunction $\Phi_{\mathbf{n} \alpha}\left(R_{\alpha}, \mathbf{r}_{\alpha}\right)$. In addition we are using the convention that wavefunction in the bra symbol $\langle/$ are not complex conjugated. This and the specific form and normalization used for the $\Phi$ 's are standard conventions in quantum scattering calculations via the S-matrix KVP[42]. It is useful to adopt this particular convention because it more explicitly reveals the symmetry of the S-matrix. $\hat{H}$ is the total Hamiltonian and $\Phi_{\mathbf{n} \alpha}$ is any regular reactive scattering wavefunction with the following asymptotic form:

$$
\begin{aligned}
\lim _{R_{\alpha} \rightarrow 0^{+}} \Phi_{\mathbf{n} \alpha}\left(R_{\alpha}, \mathbf{r}_{\alpha}\right) & =0 \\
\lim _{R_{\alpha} \rightarrow \infty} \Phi_{\mathbf{n} \alpha}\left(R_{\alpha}, \mathbf{r}_{\alpha}\right) & =-\psi_{\mathbf{n} \alpha}\left(\mathbf{r}_{\alpha}\right) \frac{e^{-i k_{\mathbf{n}} R_{\alpha}}}{\sqrt{v_{\mathbf{n} \alpha}}} \\
& +\sum_{\mathbf{n}^{\prime} \alpha^{\prime} \in \text { open }} \psi_{\mathbf{n}^{\prime} \alpha^{\prime}}\left(\mathbf{r}_{\alpha^{\prime}}^{\prime}\right) \frac{e^{i k_{\mathbf{n}^{\prime} \alpha^{\prime}} R_{\alpha^{\prime}}}}{\sqrt{v_{\mathbf{n}^{\prime} \alpha^{\prime}}}} S_{\mathbf{n}^{\prime} \alpha^{\prime}, \mathbf{n}_{\alpha}}^{0}
\end{aligned}
$$


where $\psi_{\mathbf{n} \alpha}\left(\mathbf{r}_{\alpha}\right)$ and $v_{\mathbf{n}_{\alpha}}$ are the internal eigenfunction and asymptotic translational velocity in channel $\mathbf{n} \alpha$ respectively. In Eq. (3.6), the sum is over all open channels available at energy $E$. The asymptotic form for the scattering wavefunction, physically, corresponds to a unit incoming wave in channel $\mathbf{n} \alpha$ and outgoing waves in all other open channels $\mathbf{n}^{\prime} \alpha^{\prime}$. Note that if $\Phi_{\mathbf{n} \alpha}\left(R_{\alpha}, \mathbf{r}_{\alpha}\right)$ is exact then the exact S-matrix is given by $S_{\mathbf{n}^{\prime} \alpha^{\prime}, \mathbf{n} \alpha}^{0}$.

In the Kohn variational method all of the effort is concentrated on finding a good trial wavefunction. The better the trial wavefunction the smaller is the third term $\Delta S_{\mathbf{n}^{\prime} \alpha^{\prime}, \mathbf{n} \alpha}(E)$ in Eq. (3.4) involving the full Green's function. Usually the $\Phi$ 's are chosen as a linear combination of a given basis set. The exapansion coefficients are calculated by variationally optimizing the trial wavefunction based on making the first two terms in Eq. (3.4) stationary i.e.,

$$
\delta\left[S_{\mathbf{n}^{\prime} \alpha^{\prime}, \mathbf{n} \alpha}^{0}(E)+\frac{i}{\hbar}\left\langle\Phi_{\mathbf{n}^{\prime} \alpha^{\prime}}|\hat{H}-E| \Phi_{\mathbf{n} \alpha}\right\rangle\right] \equiv \delta S_{\mathbf{n}^{\prime} \alpha^{\prime}, \mathbf{n} \alpha}^{K V P}(E)=0 .
$$

If the basis is large enough the correction term involving $\hat{G}^{+}(E)$ will be negligible because $\varphi_{\mathbf{n} \alpha} \equiv(\hat{H}-E) \Phi_{\mathbf{n} \alpha} \approx 0$. However, for sufficiently large molecular systems this will not be possible and it becomes important to consider techniques to evaluate the full Green's function.

There are many possible ways of choosing approximate scattering wavefunctions for the $\Phi$ 's. For example, we could choose partially distorted waves but we are more interested in this chapter to choose the reduced dimensionality wavefunction. This reduced dimensionality wavefunction is obtained by carrying out a quantum scattering calculation for the few most important degrees of freedom, assuming the rest to be uncoupled from them (or perhaps treating them within a vibrationally adiabatic approximation). The reduced exact calculation provides us with $S_{\mathbf{n}^{\prime} \alpha^{\prime}, \mathbf{n} \alpha}^{K V P}(E)$ which is then corrected by calculating $\Delta S_{\mathbf{n}^{\prime} \alpha^{\prime}, \mathbf{n} \alpha}(E)$ which contains all higher order corrections beyond the Kohn variational treatment. There has been a considerable amount of work on calculating $\Delta S_{\mathbf{n}^{\prime} \alpha^{\prime}, \mathbf{n} \alpha}(E)$ by quantum methods[44]. As indicated in the introduction to this chapter, the numerical bottleneck to exact quantum methods is the calculation of the full Green's function. This feature, in accordance with the conservation of difficulty principle, has limited the applications to atmost four 
atom systems. In the next section we will present a semiclassical approximation to $\Delta S_{\mathbf{n}^{\prime} \alpha^{\prime}, \mathbf{n} \alpha}(E)$ which has the potential to be applicable to larger systems.

\subsection{Semiclassical approximation to $\Delta S_{\mathbf{n}^{\prime} \alpha^{\prime}, \mathbf{n} \alpha}(E)$}

The starting point for the semiclassical approximation is to note the following exact operator relation:

$$
\hat{G}^{+}(E)=\frac{1}{i \hbar} \int_{0}^{\infty} d t \exp \left(\frac{i E t}{\hbar}\right) \exp \left(-\frac{i \hat{H} t}{\hbar}\right) .
$$

There is a crucial reason for changing over to the time representation as opposed to directly performing the semiclassical approximation in the energy representation. The reason being that time domain and energy domain are not exactly equivalent in semiclassical dynamics. In order to see this clearly let us denote the position space representation of $\hat{G}^{+}(E)$ and $e^{-i \hat{H} t / \hbar}$ by $\mathcal{G}$ and $\mathcal{K}$ respectively. The following diagram summarizes the reasoning:

$$
\begin{aligned}
& \mathcal{G}_{s c} \stackrel{S P}{\longleftarrow} \mathcal{G} \stackrel{F T}{\longleftrightarrow} \mathcal{K} \stackrel{S P}{\longrightarrow} \mathcal{K}_{s c} \\
& \mathcal{G}_{s c} \stackrel{S P F T}{\longrightarrow} \mathcal{K}_{s c}
\end{aligned}
$$

$\mathcal{G}_{s c}$ and $\mathcal{K}_{s c}$ are semiclassical counterparts of $\mathcal{G}$ and $\mathcal{K}$ obtained via stationary phase (SP) approximation. While $\mathcal{G}$ and $\mathcal{K}$ are equivalent through the Fourier transform (FT) defined in Eq. (3.8), the semiclassical counterparts are not. Instead $\mathcal{G}_{s c}$ and $\mathcal{K}_{s c}$ are equivalent under stationary phase fourier transform (SPFT). In particular semiclassical approximations in energy representation can have very different accuracies when compared to approximations in the time representation. Thus, depending on the dynamics of the system of interest, it is better to perform the semiclassical approximation in a particular representation. For example, in the case of a simple harmonic oscillator it is well known that $\mathcal{K}_{s c}$ is exact whereas $\mathcal{G}_{s c}$ is not [57]. This is the essence of the important idea put forward by Heller and coworkers[58]. Anticipating short time dynamics in our case we perform the semiclassical approximation in the time domain. 
Using relation (3.8) and inserting complete sets of position states into the correction term $\Delta S_{\mathbf{n}^{\prime} \alpha^{\prime}, \mathbf{n} \alpha}(E)$ we obtain

$$
\Delta S_{\mathbf{n}^{\prime} \alpha^{\prime}, \mathbf{n} \alpha}(E)=\frac{1}{\hbar^{2}} \int_{\mathbf{x}_{2}^{\alpha^{\prime}}, \mathbf{x}_{1}^{\alpha}, t} e^{i E t / \hbar} \varphi_{\mathbf{n}^{\prime} \alpha^{\prime}}\left(\mathbf{x}_{2}^{\alpha^{\prime}}\right) K\left(\mathbf{x}_{2}^{\alpha^{\prime}}, t ; \mathbf{x}_{1}^{\alpha}, 0\right) \varphi_{\mathbf{n} \alpha}\left(\mathbf{x}_{1}^{\alpha}\right)
$$

where we have explicitly labeled the arrangement index on the position states collectively denoted by $\mathbf{x}$ and for compactness adopted the following notation:

$$
\int_{\mathbf{x}_{2}^{\alpha^{\prime}}, \mathbf{x}_{1}^{\alpha}, t} \equiv \int_{-\infty}^{\infty} d \mathbf{x}_{2}^{\alpha^{\prime}} \int_{-\infty}^{\infty} d \mathbf{x}_{1}^{\alpha} \int_{0}^{\infty} d t
$$

The exact propagator $K\left(\mathbf{x}_{2}^{\alpha^{\prime}}, t ; \mathbf{x}_{1}^{\alpha}, 0\right)$ is defined as

$$
K\left(\mathbf{x}_{2}^{\alpha^{\prime}}, t ; \mathbf{x}_{1}^{\alpha}, 0\right) \equiv\left\langle\mathbf{x}_{2}^{\alpha^{\prime}}\left|\exp \left(-\frac{i \hat{H} t}{\hbar}\right)\right| \mathbf{x}_{1}^{\alpha}\right\rangle
$$

The exact propagator can be formally defined via Feynman path integrals[62]. A path integral analysis of the correction term can be performed leading us to the standard theory of reactive scattering based on path integrals $[9,11,63]$. However, we are interested in a semiclassical approximation for the propagator. This can be achieved by doing a stationary phase analysis on the path integral or through the traditional WKB approach. Both approaches result in the celebrated Van VleckGutzwiller approximation[64] for the exact propagator ${ }^{1}$

$$
\begin{aligned}
K\left(\mathbf{x}_{2}^{\alpha^{\prime}}, t ; \mathbf{x}_{1}^{\alpha}, 0\right) \approx & K_{s c}\left(\mathbf{x}_{2}^{\alpha^{\prime}}, t ; \mathbf{x}_{1}^{\alpha}, 0\right) \\
=\left(\frac{1}{2 \pi i \hbar}\right)^{F / 2} \sum_{k} \mid & \left.\operatorname{Det}\left(\frac{\partial^{2} \mathcal{S}_{k}\left(\mathbf{x}_{2}^{\alpha^{\prime}}, \mathbf{x}_{1}^{\alpha} ; t\right)}{\partial \mathbf{x}_{2}^{\alpha^{\prime}} \partial \mathbf{x}_{1}^{\alpha}}\right)\right|^{1 / 2} \\
& \times \exp \left(\frac{i \mathcal{S}_{k}\left(\mathbf{x}_{2}^{\alpha^{\prime}}, \mathbf{x}_{1}^{\alpha} ; t\right)}{\hbar}-\frac{i \pi \mu_{k}}{2}\right) .
\end{aligned}
$$

In Eq. (3.13) the Jacobian determinant plays the role of a classical probability and the phase is determined by the classical action $\mathcal{S}_{k}\left(\mathbf{x}_{2}^{\alpha^{t}}, \mathbf{x}_{1}^{\alpha} ; t\right) . F$ is the number of degrees of freedom. The classical action for the $k$ th trajectory that goes from $\mathbf{x}_{1}^{\alpha}$

\footnotetext{
${ }^{1}$ We are assuming that the Hamiltonian is of cartesian form. This is true for one degree of freedom system and collinear atom-diatom collisions. For non-cartesian systems it is straightforward to work out the relevant expression through point coordinate canonical transformations.
} 
at time $t=0$ to $\mathbf{x}_{2}^{\alpha^{t}}$ at time $t$ is given by the time integral of the corresponding Lagrangian $\mathcal{L}$,

$$
\begin{aligned}
\mathcal{S}_{k}\left(\mathbf{x}_{2}^{\alpha^{\prime}}, \mathbf{x}_{1}^{\alpha} ; t\right) & =\int_{0}^{t} d t^{\prime} \mathcal{L}\left(\mathbf{x}, \dot{\mathbf{x}} ; t^{\prime}\right) \\
& =\int_{0}^{t} d t^{\prime}\left[\mathbf{p}\left(t^{\prime}\right) \cdot \dot{\mathbf{x}}\left(t^{\prime}\right)-\mathcal{H}\left(\mathbf{p}\left(t^{\prime}\right), \mathbf{x}\left(t^{\prime}\right)\right)\right]
\end{aligned}
$$

where $\mathcal{H}$ is the classical Hamiltonian and the sum over $k$ in Eq. (3.13) is over all possible classical trajectories that satisfy this double ended boundary condition. The Maslov index ${ }^{2} \mu_{k}$ is given by the number of zeros experienced by the inverse of the Jacobian determinant in the time interval $(0, t)[60]$. Sustituting the semiclassical propagator into Eq. (3.10) we obtain the semiclassical approximation for $\Delta S_{\mathbf{n}^{\prime} \alpha^{\prime}, \mathbf{n} \alpha}(E)$

$$
\begin{aligned}
\Delta S_{\mathbf{n}^{\prime} \alpha^{\prime}, \mathbf{n} \alpha}^{s c}(E) & =\frac{(2 \pi i \hbar)^{-F / 2}}{\hbar^{2}} \\
& \times \sum_{k} \int_{\mathbf{x}_{2}^{\alpha^{\prime}}, \mathbf{x}_{1}^{\alpha}, t} \mathcal{A}_{\mathbf{n}^{\prime} \alpha^{\prime}, \mathbf{n} \alpha}^{(k)}\left(\mathbf{x}_{2}^{\alpha^{\prime}}, \mathbf{x}_{1}^{\alpha} ; t\right) \exp \left(\frac{i \mathcal{P}^{(k)}\left(\mathbf{x}_{2}^{\alpha^{\prime}}, \mathbf{x}_{1}^{\alpha} ; t\right)}{\hbar}\right),
\end{aligned}
$$

where

$$
\begin{aligned}
\mathcal{A}_{\mathbf{n}^{\prime} \alpha^{\prime}, \mathbf{n} \alpha}^{(k)}\left(\mathbf{x}_{2}^{\alpha^{\prime}}, \mathbf{x}_{1}^{\alpha} ; t\right) & \equiv\left|\operatorname{Det}\left(\frac{\partial^{2} \mathcal{S}_{k}\left(\mathbf{x}_{2}^{\alpha^{\prime}}, \mathbf{x}_{1}^{\alpha} ; t\right)}{\partial \mathbf{x}_{2}^{\alpha^{\prime}} \partial \mathbf{x}_{1}^{\alpha}}\right)\right|^{1 / 2} \varphi_{\mathbf{n}^{\prime} \alpha^{\prime}}\left(\mathbf{x}_{2}^{\alpha^{\prime}}\right) \varphi_{\mathbf{n} \alpha}\left(\mathbf{x}_{1}^{\alpha}\right) \\
\mathcal{P}^{(k)}\left(\mathbf{x}_{2}^{\alpha^{\prime}}, \mathbf{x}_{1}^{\alpha} ; t\right) & \equiv \mathcal{S}_{k}\left(\mathbf{x}_{2}^{\alpha^{\prime}}, \mathbf{x}_{1}^{\alpha} ; t\right)+E t-\frac{\pi \hbar \mu_{k}}{2} .
\end{aligned}
$$

In Eq. (3.15) we still have to sum over all trajectories that go from $x_{1}^{\alpha}$ to $x_{2}^{\alpha^{\prime}}$ in time $t$. For a given system there are, in general, many such trajectories and we have to search for them all in the full phase space of the problem. This root search problem for the double ended boundary conditions can become prohibitive for large systems. A way to avoid the root search problem is to transform to an initial value representation. It is well known in classical mechanics that a classical trajectory with a given initial condition is unique[19]. Thus for fixed $\mathbf{x}_{1}^{\alpha}$ and $t$ we change the integration variable from $\mathbf{x}_{2}^{\alpha^{\prime}}$ to $\mathbf{p}_{1}^{\alpha}$,

$$
\sum_{k} d \mathbf{x}_{2}^{\alpha^{\prime}} \longrightarrow\left|\operatorname{Det}\left(\frac{\partial \mathbf{x}_{2}^{\alpha^{\prime}}\left(\mathbf{x}_{1}^{\alpha}, \mathbf{p}_{1}^{\alpha} ; t\right)}{\partial \mathbf{p}_{1}^{\alpha}}\right)\right| d \mathbf{p}_{1}^{\alpha},
$$

\footnotetext{
${ }^{2}$ Van Vleck originally wrote down the semiclassical approximation in 1928 without the sum over $k$ or the Maslov index. This limited the approximation to very short times. The more accurate form, as presented in this section, was worked out by Gutzwiller.
} 
where the sum over root trajectories is subsumed in the integration over $\mathbf{p}_{1}^{\alpha}$. Notice that we have not solved the root search problem but only circumvented it by going to an initial value representation. We have to run classical trajectories in the full phase space in order to perform the root search and sum over the root trajectories. In the initial value representation we are making use of all the trajectories and thus implementing the root search in a straightforward fashion. There is a price to be paid i.e., now we are running more trajectories then the number of root trajectories. Nevertheless, it can be argued that this extra cost is offset by the efforts required to determine the root trajectories. This is especially true for large systems for which we are interested in applying this model. Using the relation

$$
\frac{\partial \mathcal{S}\left(\mathbf{x}_{2}^{\alpha^{\prime}}, \mathbf{x}_{1}^{\alpha} ; t\right)}{\partial \mathbf{x}_{1}^{\alpha}}=-\mathbf{p}_{1}^{\alpha}
$$

and substituting into Eq. (3.15) we obtain the semiclassical correction term in the initial value representation as

$$
\begin{aligned}
\Delta S_{\mathbf{n}^{\prime} \alpha^{\prime}, \mathbf{n} \alpha}^{s c}(E) & =\frac{(2 \pi i \hbar)^{-F / 2}}{\hbar^{2}} \\
& \times \int_{\mathbf{x}_{1}^{\alpha}, \mathbf{p}_{1}^{\alpha}, t} \mathcal{A}_{\mathbf{n}^{\prime} \alpha^{\prime}, \mathbf{n} \alpha}\left(\mathbf{x}_{2}^{\alpha^{\prime}}, \mathbf{x}_{1}^{\alpha} ; t\right) \exp \left(\frac{i \mathcal{P}\left(\mathbf{x}_{2}^{\alpha^{\prime}}, \mathbf{x}_{1}^{\alpha} ; t\right)}{\hbar}\right),
\end{aligned}
$$

where

$$
\begin{aligned}
\mathcal{A}_{\mathbf{n}^{\prime} \alpha^{\prime}, \mathbf{n} \alpha}\left(\mathbf{x}_{2}^{\alpha^{\prime}}, \mathbf{x}_{1}^{\alpha} ; t\right) & \equiv\left|\operatorname{Det}\left(\frac{\partial \mathbf{x}_{2}^{\alpha^{\prime}}}{\partial \mathbf{p}_{1}^{\alpha}}\right)\right|^{1 / 2} \varphi_{\mathbf{n}^{\prime} \alpha^{\prime}}\left(\mathbf{x}_{2}^{\alpha^{\prime}}\right) \varphi_{\mathbf{n} \alpha}\left(\mathbf{x}_{1}^{\alpha}\right) \\
\mathcal{P}\left(\mathbf{x}_{2}^{\alpha^{\prime}}, \mathbf{x}_{1}^{\alpha} ; t\right) & \equiv \mathcal{S}\left(\mathbf{x}_{2}^{\alpha^{\prime}}, \mathbf{x}_{1}^{\alpha} ; t\right)+E t-\frac{\pi \hbar \mu}{2} \\
\mathbf{x}_{2}^{\alpha^{\prime}} & \equiv \mathbf{x}_{2}^{\alpha^{\prime}}\left(\mathbf{x}_{1}^{\alpha}, \mathbf{p}_{1}^{\alpha} ; t\right)
\end{aligned}
$$

The Maslov index $\mu$ is now given by the number of zeros experienced by the determinant in the time interval $(0, t)$. The matrix occuring in Eq. (3.20) is part of a $2 F \times 2 F$ matrix called as the monodormy matrix ${ }^{3}$ which provides information about the stability of nearby classical trajectories in phase space. The time evolution of the monodormy matrix $\mathcal{M}$ is given by:

$$
\frac{d}{d t} \mathcal{M}(t)+\mathcal{F}(t) \cdot \mathcal{M}(t)=0
$$

\footnotetext{
${ }^{3}$ This also goes by various other names in the literature. For example, stability matrix, Whittaker's matrix etc. $\mathcal{M}$ is invariant under canonical transformations.
} 
where we have denoted

$$
\mathcal{M}(t)=\frac{\partial\left(\mathbf{x}_{2}^{\alpha^{\prime}}, \mathbf{p}_{2}^{\alpha^{\prime}}\right)}{\partial\left(\mathbf{x}_{1}^{\alpha}, \mathbf{p}_{1}^{\alpha}\right)}(t),
$$

and $\mathcal{F}$ is a generalized force constant matrix

$$
\mathcal{F}(t)=\left(\begin{array}{cc}
-\partial_{\mathbf{p x}} \mathcal{H} & -\partial_{\mathbf{p p}} \mathcal{H} \\
\partial_{\mathbf{x x}} \mathcal{H} & \partial_{\mathbf{x p}} \mathcal{H}
\end{array}\right) .
$$

The coupled equations of motion in Eq. (3.21) are integrated along with the usual Hamilton's equations of motion with the initial conditions

$$
\mathcal{M}(0)=\left(\begin{array}{ll}
1 & 0 \\
0 & 1
\end{array}\right) .
$$

Thus the Maslov index is kept track of along the classical trajectory.

Eq. (3.19) is the fundamental result of this chapter. There are a few significant features of this result that we wish to emphasize. Firstly, classical S-matrix theory is obatined from Eq. (3.19) if the integral over initial phase space variables is performed via stationary phase approximation. Here, however, we are interested in evaluating them exactly (numerically) as discussed in the beginning of the section. The functions $\varphi_{\mathbf{n}^{\prime} \alpha^{\prime}}\left(\mathbf{x}_{2}^{\alpha^{\prime}}\right)$ and $\varphi_{\mathbf{n} \alpha}\left(\mathbf{x}_{1}^{\alpha}\right)$ are decaying rapidly (as $\mathcal{L}^{2}$ functions) for large $\mathbf{x}_{1}^{\alpha}$ and $\mathbf{x}_{2}^{\alpha^{\prime}}$. This implies that only relatively short time dynamics is required and hence the reason for performing the semiclassical approximation in the time domain. Thus we expect the approximation to have an even better chance of being usefully accurate then the generic long time accuracy indicated by Heller's recent work.

Secondly, tunneling contributions are calculated via real time classical trajectories only. In order to see this, concentrate on the time integral. The trajectory that corresponds to zero time is the one with an infinite amount of energy ${ }^{4}$-a trajectory far above the barrier. On the other hand, the classical trajectory that corresponds to infinite time is the separatrix trajectory i.e., one which just skims the top of the barrier. Hence if we do all the integrals numerically exactly then all of the tunneling

\footnotetext{
${ }^{4}$ This trajectory energy $\mathcal{E}$ is different from the energy $E$ at which we are calculating the $S$ matrix. Since we are in the time representation, we are summing over all trajectories with different energies. Stationary phase result would only sum up contributions from trajectories which satisfy $\mathcal{E}\left(\mathbf{x}_{1}^{\alpha}, \mathbf{p}_{1}^{\alpha}\right)=E$.
} 
contributions are included in the real time trajectories with energies above the barrier. The tunneling contributions are small and it will be demonstarted in the next section that it is indeed possible to calculate these small numbers relatively well with real valued classical trajectories.

Finally, in performing the integrals in Eq. (3.19) exactly, we are not consistent to $O(\hbar)$ anymore. This implies that we lose canonical invariance to $O(\hbar)$ with respect to certain parameters in our system which we would have if stationary phase was the method of choice for integration. In other words, our results would have subtle dependencies on some parameters which, otherwise, would not be expected. For example, in the next section it will be seen that the amplitudes and hence probabilities vary with a parameter assosciated with the trial wavefunction although the exact results do not depend on this parameter. In some sense, the stability of the results under the variation of the parameter would be an indication of the robustness of the semiclassical approximation. For harmonic systems we still have canonical invariance as stationary phase result is exact in this case.

\subsection{Application to a model potential}

In this section we will use the following notation:

$$
\begin{array}{lll}
h(x) \equiv h\left(-x-x_{0}\right) & \bar{h}(x) \equiv h\left(x-x_{0}\right) \\
\delta(x) \equiv \delta\left(-x-x_{0}\right) & \bar{\delta}(x) \equiv \delta\left(x-x_{0}\right),
\end{array}
$$

where $h(z)$ is a smooth step function ( $=1$ for $z \gg 0$, and 0 for $z \ll 0)$ and $\delta(z)$ is the corresponding smooth pre-limit version of a Dirac delta function. $x_{0}$ denotes the position of the smooth step function. For the applications below we used

$$
\begin{aligned}
h(z) & =\frac{1}{1+e^{-\lambda z}} \\
\delta(z) & =\frac{\lambda}{4} \operatorname{sech}^{2}\left(\frac{\lambda z}{2}\right) .
\end{aligned}
$$

We apply the ideas of the previous section on a one dimensional model of a 
chemical reaction, namely transmission through an Eckart barrier[65],

$$
V(x)=V_{0} \operatorname{sech}^{2}(a x)
$$

The barrier height $V_{0}$ and mass $m$ were chosen to be $0.01562 a u(\approx 0.425 \mathrm{eV})$ and $1060 a u$ respectively. The parameter $a$, related to the anharmonicity of the barrier, is set equal to $1.3624 \mathrm{au}$. With this choice of parameters, the potential approximately corresponds to the collinear $H+H_{2}$ reaction.

The Eckart barrier is the simplest model of chemical reaction but not completely trivial. Furthermore, in order to provide the most stringent test of the semiclassical approximation, we make the simplest possible choice for the trial wavefunctions as smoothly cutoff plane waves,

$$
\begin{aligned}
& \Phi_{n}(x)=\frac{e^{i k x}}{\sqrt{v}} h(x) \\
& \Phi_{\bar{n}}(x)=\frac{e^{-i k x}}{\sqrt{v}} \bar{h}(x),
\end{aligned}
$$

where $n$ represents reactants to the left and $\bar{n}$ represents products to the right of the barrier. $v$ and $k$ are the asymptotic velocity and wavevector respectively, given by the usual relation $v=\hbar k / m=\sqrt{2 E / m}$. Thus $\Phi_{n}(x)$ is cutoff smoothly on the left side of the barrier at $x \approx-x_{0}$, so that

$$
\begin{aligned}
& \Phi_{n}(x) \approx \frac{e^{i k x}}{\sqrt{v}}, \quad x \ll-x_{0} \\
& \Phi_{n}(x) \approx 0, \quad x \gg-x_{0}
\end{aligned}
$$

and similarly for $\Phi_{\bar{n}}(x)$ at $x \approx x_{0}$. Note that in our choice of the trial wavefunctions there is no distortion and thus corresponds to the simplest Kohn calculation for our system. As a result, the semiclassical correction term is rather large. With this choice for the trial wavefunctions the zeroth order term in Eq. (3.4) is zero and the Kohn part of the S-matrix is given by

$$
\begin{aligned}
S_{\bar{n}, n}^{K V P}(E) & =\frac{i}{\hbar}\left\langle\Phi_{\bar{n}}|\hat{H}-E| \Phi_{n}\right\rangle \\
& =\frac{i}{\hbar v} \int_{x}\left[V(x) \bar{h}(x) h(x)+i \hbar v \bar{h}(x) \delta(x)-\frac{\hbar^{2}}{2 m} \bar{\delta}(x) \delta(x)\right],
\end{aligned}
$$


where we have used the result

$$
\varphi_{n}(x)=\frac{e^{i k x}}{\sqrt{v}}\left[V(x) \bar{h}(x)+i \hbar v \bar{\delta}(x)+\frac{\hbar^{2}}{2 m} \frac{d \bar{\delta}(x)}{d x}\right],
$$

and similarly for $\varphi_{\bar{n}}(x)$. In this case we can write down the explicit form of the semiclassical correction term as

$$
\Delta S_{\tilde{n}, n}^{s c}(E)=\frac{(2 \pi \hbar)^{-1 / 2}}{\hbar^{2} v} \int_{x_{1}, p_{1}, t} \mathcal{A}\left(x_{1}, p_{1} ; t\right) \exp \left(\frac{i \mathcal{P}\left(x_{1}, p_{1} ; t\right)}{\hbar}\right)
$$

where

$$
\begin{aligned}
\mathcal{A}\left(x_{1}, p_{1} ; t\right) & \equiv\left|\frac{\partial x_{2}}{\partial p_{1}}\right|^{1 / 2}\left[V\left(x_{1}\right) h\left(x_{1}\right)+\frac{i \hbar}{2}\left(v+\frac{p_{1}}{m}\right) \delta\left(x_{1}\right)\right] \\
\times\left[V\left(x_{2}\right) \bar{h}\left(x_{2}\right)+\frac{i \hbar}{2}\left(v+\frac{p_{2}}{m}\right) \bar{\delta}\left(x_{2}\right)\right] & \\
\mathcal{P}\left(x_{1}, p_{1} ; t\right) & \equiv \hbar k\left(x_{1}-x_{2}\right)+\mathcal{S}\left(x_{2}, x_{1} ; t\right)+E t-\frac{\pi \hbar}{2}\left(\mu+\frac{1}{2}\right), \\
x_{2} & =x_{2}\left(x_{1}, p_{1} ; t\right), \quad p_{2}=p_{2}\left(x_{1}, p_{1} ; t\right) .
\end{aligned}
$$

The integral over time in Eq. (3.32) is evaluated simultaneously with computing the trajectory with initial conditions $\left(p_{1}, x_{1}\right)$. The behaviour of the integrand with respect to the initial phase space variables is not completely smooth. As expected, the integrand as a function of $p_{1}$ for fixed $x_{1}$ is oscillatory. Figure (3.1) shows a typical plot of the integrand as a function of $p_{1}$.

For $E>V_{0}$ there is a stationary phase region in the integrand which corresponds to the classical trajectory with energy $\mathcal{E}\left(p_{1}, x_{1}\right)=E$. In the case of $E<V_{0}$ there is no real $\left(p_{1}, x_{1}\right)$ such that $\mathcal{E}\left(p_{1}, x_{1}\right)=E$ and, not surprisingly, we do not see a stationary phase region. On studying the integrand in Eq. (3.32) as a function of $p_{1}$ for a given $x_{1}$ we observe a singularity at some sharp value $p_{1}=p_{1}^{s p}$. Analyzing the integrand more carefully revealed that the singular point corresponded to the separatrix trajectory i.e., $\mathcal{E}\left(p_{1}^{s p}, x_{1}\right)=V_{0}$ and more importantly that it is a removable singularity ${ }^{5}$. In order for the numerical integration to be smooth, we transformed from $p_{1}$ to a variable $\tilde{p}_{1}$

$$
p_{1}=\tilde{p}_{1}^{2}+p_{1}^{s p}
$$

\footnotetext{
${ }^{5}$ Please see appendix for a theoretical analysis of the singularity assosciated with the separatrix trajectory.
} 


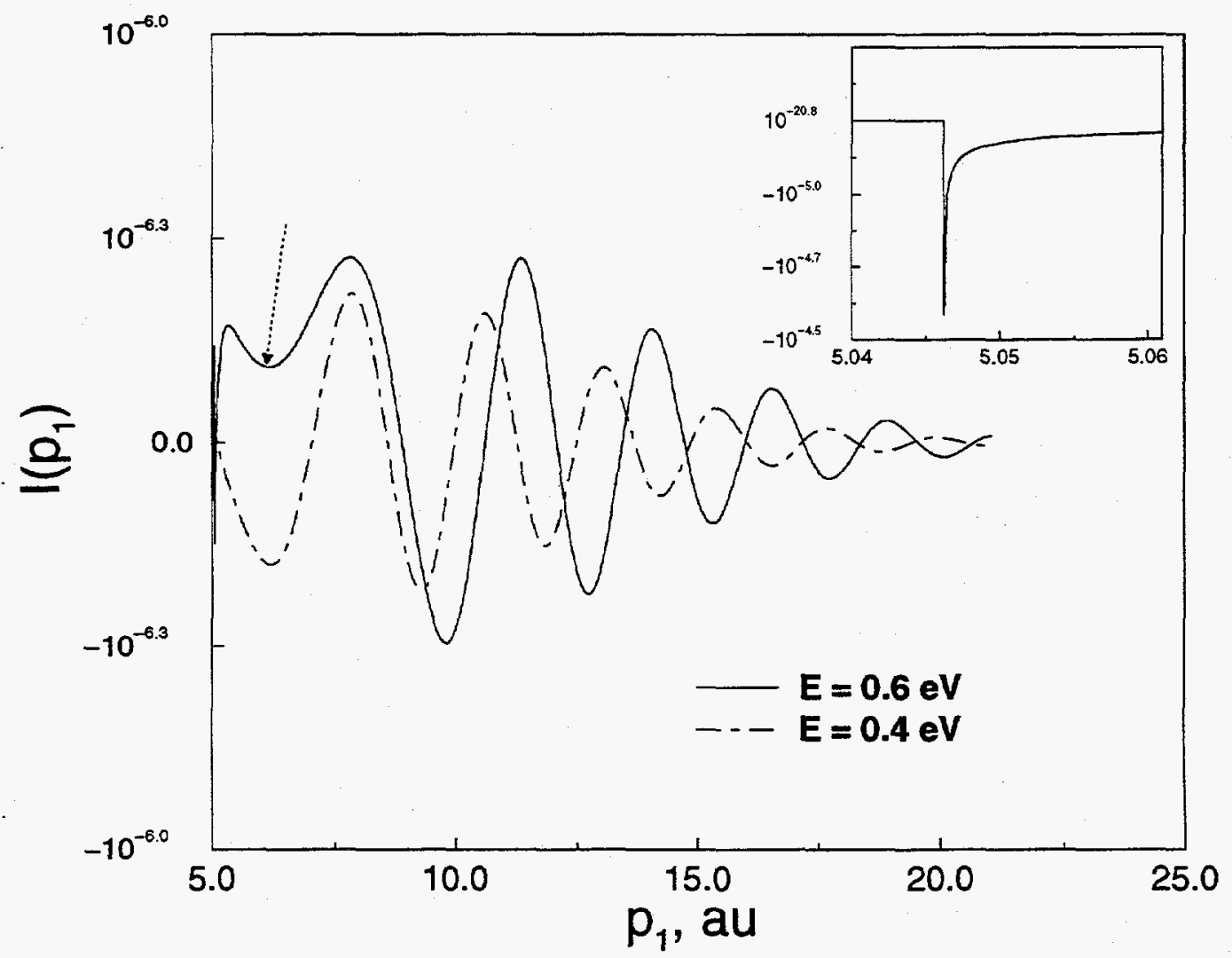

Figure 3.1: A typical integrand in Eq. (3.32) is shown here as a function of $p_{1}$ for a fixed value of $x_{1}$ and the parameters $x_{0}, \lambda$. Barrier height $V_{0}=0.425 \mathrm{eV}$. Notice the stationary phase region for $E>V_{0}$, indicated in the plot by an arrow. The inset shows the singularity in the integrand corresponding to the separatrix trajectory. 
where $p_{1}^{s p}$ is the separatrix momentum, determined for a given $x_{1}$ as

$$
\begin{aligned}
p_{1}^{s p} & =\sqrt{\left[2 m\left(V_{0}-V\left(x_{1}\right)\right]\right.} \\
& =\sqrt{2 m V_{0}} \tanh \left(a x_{1}\right) .
\end{aligned}
$$

\subsubsection{Results and discussion}

Figure (3.2) shows the transmission probability $P(E) \equiv\left|S_{\bar{n}, n}(E)\right|^{2}$ as a function of energy for the cutoff parameter $x_{0}=5 \mathrm{au}$. For comparison we have also shown the exact[66] and WKB[13] results for the probability

$$
\begin{aligned}
P_{e x}(E) & =\left\{1+[\cosh (c) / \sinh (b)]^{2}\right\}^{-1} \\
P_{w k b}(E) & =\left(1+e^{2 \theta(E)}\right)^{-1}
\end{aligned}
$$

with

$$
\begin{aligned}
\theta(E) & =-b+\sqrt{c^{2}+\frac{\pi^{2}}{4}} \\
b & =\pi \sqrt{\frac{2 m E}{(a \hbar)^{2}}}, \quad c=\frac{\pi}{2} \sqrt{\frac{8 V_{0} m}{(a \hbar)^{2}}-1}
\end{aligned}
$$

Our choice for $x_{0}$ implies that we have cutoff the $\Phi$ 's well into their respective asymptotic regions. Thus in the interaction region (where the potential is significantly larger than its asymptotic value of zero) the trial wavefunctions $\Phi_{\bar{n}}(x)$ and $\Phi_{n}(x)$ have essentially zero overlap. As a result of this the Kohn variational term in Eq. (3.30) vanishes. Thus the entire contribution to the transmission probability is from the semiclassical correction term $\Delta S_{\tilde{n}, n}^{s c}(E)$. It is impressive to see how well the semiclassical approximation does even in the deep tunneling region. For the values of $P(E)$ below $10^{-6}$ the results become progressively worse, being about an order of magnitude too large when $P \approx 10^{-7}$. In this one dimensional example, the WKB results do better than the present semiclassical model. However, this is to be expected as WKB is quite accurate for one dimensional systems. The important point to be made here is that the present semiclassical model is applicable to systems with many degrees of freedom whereas, there is at present no consistent WKB theory for such systems. Note that all the trajectories that contribute in Eq. (3.32) go from $x_{1} \leq-x_{0}$ 


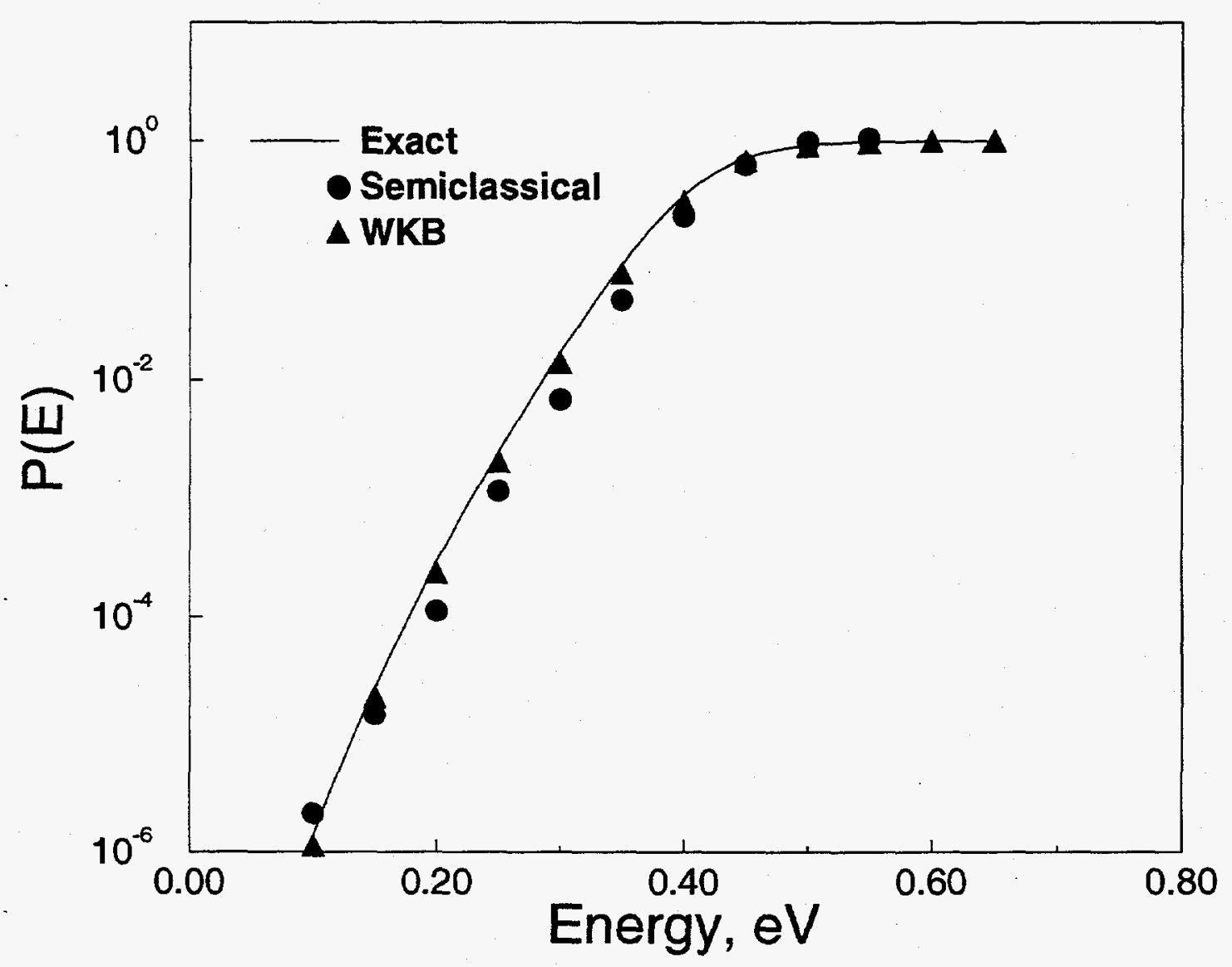

Figure 3.2: Transmission probability, $P(E)$, is shown as a function of the total energy $E$. The cutoff parameters are $\lambda=2.5 \mathrm{au}$ and $x_{0}=5.0 \mathrm{au}$. Also shown are the exact results and the results from a one dimensional WKB analysis. For this potential $E=0.2 \mathrm{eV}$ already corresponds to deep tunneling regime. 


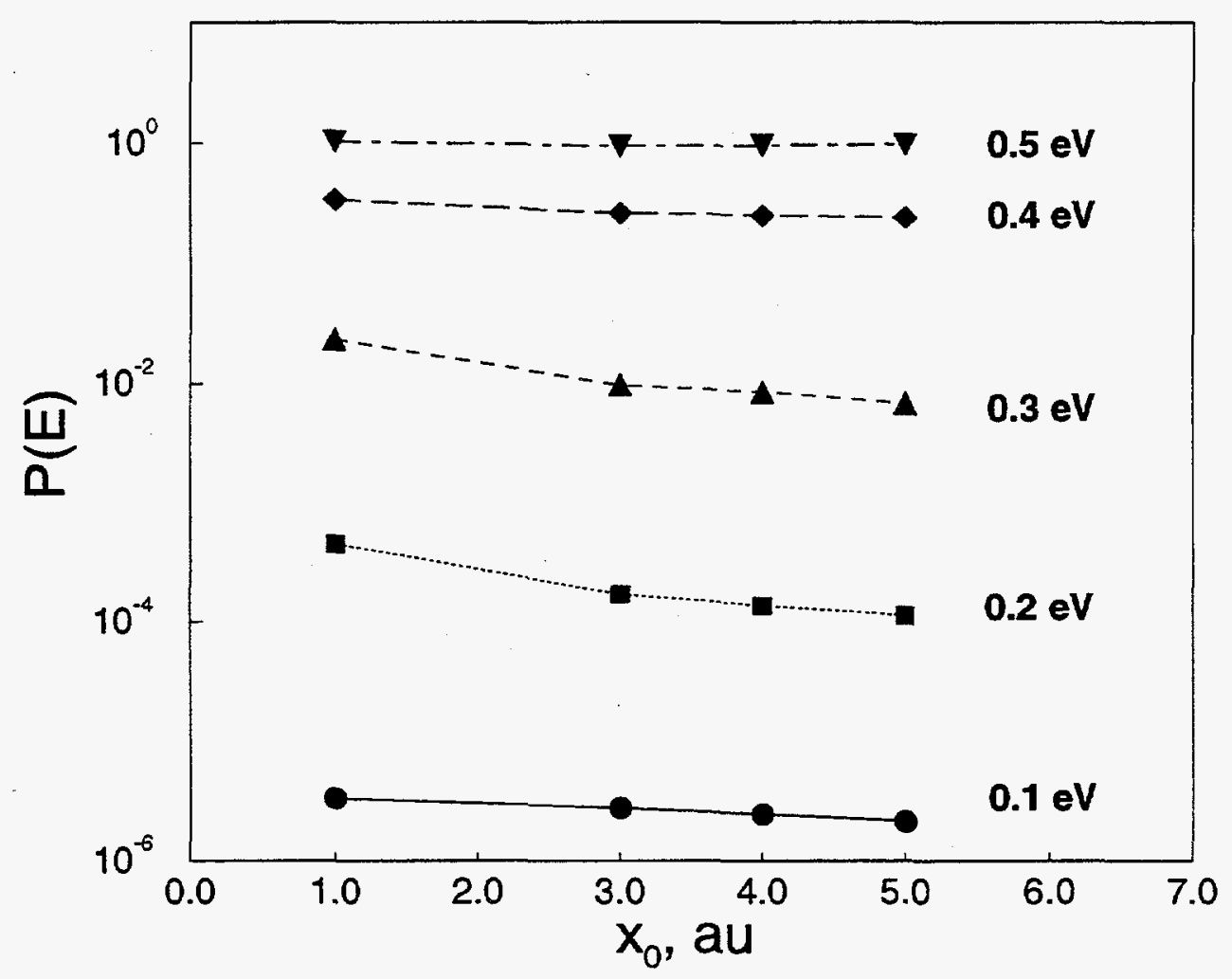

Figure 3.3: Shown here is the variation of $P(E)$ with the cutoff parameter $x_{0}$ for several representative values of the energy $E$. The parameter $\lambda$ is fixed at $2.5 \mathrm{au}$. The barrier height is about $0.425 \mathrm{eV}$. Notice the stability of the results with respect to $x_{0}$.

to $x_{2} \geq x_{0}$ in real time $t$. Thus we are getting tunneling probabilities from purely real time trajectories.

As indicated in the previous section, it is important to verify the stability of these results with respect to the parameters in order to make sure that we did not have a special set of parameters. Figure (3.3) shows how the probabilities vary with respect to the cutoff parameter $x_{0}$. If the semiclassical approximation were exact, $P(E)$ would be independent of $x_{0}$ and of the particular choice of $\Phi$ 's in general. The results vary only modestly for energies above and below the barrier height over a wide range of $x_{0}$. This stability of the results with respect to variations in $x_{0}$ is an indication of the robustness of the semiclassical approximation. In these calculations, for small $x_{0}$ the Kohn variational term becomes non-negligible and has to be taken into 
account. Similar stability is also seen with respect to variations in the parameter $\lambda$. It is relatively easy to verify that the small numbers obtained for tunneling probabilities are indeed correct. This can be achieved by performing $\hbar$-scaling caculations on the system and demonstrating that the small numbers scale exponentially with $\hbar$ as

$$
P \sim e^{- \text {const } / \hbar},
$$

which is a familiar result for tunneling probabilites[37, 67]. Performing such a calculation on our system we see scaling of the form in Eq. (3.38).

\subsection{Concluding remarks}

The semiclassical hybrid approach described in this chapter shows promise of applicability to large systems. The test case of a simple but nontrivial one-dimensional potential demonstrates the quantitative and qualitative abilities of this approach. For the application described in the previous section, the semiclassical method was pushed to its limits by our choice of the parameters and trial wavefunctions. The results even in these extreme limits were quite good. Clearly, the results can be significantly improved by using better trial wavefunctions i.e., distorted waves which would be desirable when dealing with multidimensional systems. It is important to emphasize that this approach is much more theoretically self-consisitent than many other approaches considered before.

This chapter was concerned mainly with calculating the state-to-state Smatrix elements which is a very detailed quantity. It is natural to enquire if there are similar semiclassical approaches within which we can directly calculate $N(E)$ and $k(T)$. There is already a way to calculate $N(E)$ directly using absorbing boundary conditions[69]. There is also a half-state-selected version of the theory presented in this chapter. However, unlike in the exact quantum calculations, there does not seem to be any significant savings in performing a direct calculation as opposed to a stateto-state calculation. Further studies are required in order to see if it is possible to obtain accurate results with a much smaller number of trajectories than that needed for a full S-matrix calculation. The case of $k(T)$ is much more difficult and challenging. 
For some of the difficulties and a new transition state result, see appendix II of this chapter.

Some of the features resulting from this approach are worth mentioning. Historically, stationary phase approximations have always been the method of choice for highly oscillatory integrals. Indeed the stationary phase method is the main tool of analysis in most of the classic studies in semiclassical methods. There has been some work in the area of going beyond stationary phase approximation i.e., beyond $O(\hbar)$ but the asymptotic nature of the expansions limit their practical utility[13]. It was realised that the stationary phase approach to classically forbidden processes necessiates the use and understanding of complex valued classical trajectories. This work, in stark contrast from earlier studies, shows that it is possible to go beyond stationary phase and to deal with the highly oscillatory integrals numerically. This is made possible by gaining a deeper understanding of the wide variety of classical trajectories which constitute the phase space of the system. For example, one of the key feature in the integrals, manifesting itself in the form of a singularity, was attributed to the separatrix trajectory. Once we had isolated the family of trajectories leading to this behaviour it was realtively simple to alleviate the problem. A similar analysis by Tomsovic and Heller enabled them to go beyond stationary phase in a system whose classical dynamics exhibits hard chaos and to establish semiclassical accuracies for much longer times than previously considered possible[68]. Thus, within our approach, we were able to calculate tunneling probabilities with purely real-time classical trajectories. This is an important step forward as far as applicability to larger systems is concerned due to the fact that computing complex valued classical trajectories for such systems is extremely difficult. More importantly, our approach also provides a consistent way to take into account both classically allowed and forbidden processes in multidimensions. There is no clear generalization of WKB to multidimensional problems which can do this in a practical way.

On the numerical side it becomes important to consider the total number of trajectories needed in order to obtain the results provided in the previous section. In any numerical approach, quantum or semiclassical, in addition to the scaling with the system size there is also a prefactor. Although the scaling for classical trajectories 
only increases linearly with the number of degrees of freedom, the prefactor might become uncomfortably large. In order to study this more carefully, we optimized the problem and found that the prefactor in the one dimensional case is very reasonable. Another attractive numerical feature is that for all the energies of interest, a single set of trajectories is all that is needed. Thus it is possible to parallelize the computation and a single run gives us the entire S-matrix over the required energy range. This is a highly desirable feature for multidimensional systems and we are thus guaranteed of a reasonable prefactor for our numerical calculations.

In light of the above remarks, it is clear that this is an approach worthy of further studies and applications to truly complex chemical systems.

\subsection{Appendix I: Analysis of the singularity in the integrand of Eq. (3.28)}

In this appendix we will show that the singularity in the integrand of Eq. (3.32) is assosciated with the separatrix trajectory. The form of the singularity, generic to all one dimensional barrier potentials, demonstrates that it is removable via the transformation given by Eq. (3.34).

To begin with, we will cast Eq. (3.32) into a simpler but equivalent form. Trajectories are evolved from a fixed initial position $\left(x_{1}=-\bar{x}\right)$ to a fixed final position $\left(x_{2}\left(x_{1}, p_{1} ; t\right)=\bar{x}\right)$. The value of $\bar{x}$ is chosen such that it corresponds to the asymptotic region of the potential i.e., $V(\bar{x}) \approx 0$. As a result, the time integral can be trivially done due to the fact that for a given $\bar{x}$ and $p_{1}$ the time $\bar{t}$ is fixed by the relation $x_{2}\left(-\bar{x}, p_{1} ; \bar{t}\right)=\bar{x}$. Thus we are left with an integral over initial momenta only. The final expression for the S-matrix in this sharp cutoff limit is

$$
S_{\bar{n}, n}^{s c}(E)=v \int_{p_{1}} \mathcal{A}\left(-\bar{x}, p_{1} ; \bar{t}\right) \exp \left(\frac{i \mathcal{P}\left(-\bar{x}, p_{1} ; \bar{t}\right)}{\hbar}\right),
$$

where

$$
\begin{aligned}
\mathcal{A}\left(-\bar{x}, p_{1} ; \bar{t}\right) & \equiv \frac{m}{\left|p_{2}\right|}\left(\frac{1}{2 \pi \hbar}\left|\frac{\partial x_{2}}{\partial p_{1}}\right|_{\bar{x}}\right)^{1 / 2} \\
\mathcal{P}\left(-\bar{x}, p_{1} ; \bar{t}\right) & \equiv-2 \hbar k \bar{x}+E \bar{t}+\mathcal{S}\left(-\bar{x}, p_{1} ; \bar{t}\right),
\end{aligned}
$$


and now $\bar{t}=\bar{t}\left(\bar{x}, p_{1}\right)$ with the final momentum $p_{2}$ determined as a function of $\left(\bar{x}, p_{1}\right)$.

It is easy to check that for a free particle, Eq. (3.39) gives unity for the S-matrix. In order to see the singularity, let us consider a parabolic barrier

$$
V(x)=-\frac{1}{2} m \omega x^{2}
$$

The Eckart barrier, treated in the previous section, can be closely approximated by the parabolic barrier near the maximum by appropriately choosing $\omega$. For the discussion in this section the parabolic barrier example will suffice since the structure of the singularity depends on the form of the barrier. Thus any one dimensional potential with a quadratic (parabolic) barrier will give rise to the singularity. The relevant quantities for Eq. (3.39) can be worked out analytically in this case and we find

$$
\begin{aligned}
\mathcal{S}\left(\bar{x}, p_{1} ; \bar{t}\right) & =\bar{x} p_{1} \\
\bar{t}\left(\bar{x}, p_{1}\right) & =\frac{1}{\omega} \ln \left(\frac{p_{1}+p_{1}^{s p}}{p_{1}-p_{1}^{s p}}\right) \\
p_{2}\left(\bar{x}, p_{1} ; \bar{t}\right) & =p_{1} \\
\frac{\partial x_{2}}{\partial p_{1}} & =\frac{\sinh (\omega \bar{t})}{m \omega}=\frac{2 p_{1} \bar{x}}{\left(p_{1}+p_{1}^{s p}\right)\left(p_{1}-p_{1}^{s p}\right)} .
\end{aligned}
$$

The separatrix momentum is denoted by $p_{1}^{s p}=m \omega \bar{x}$ and for the parabolic barrier corresponds to an energy equal to zero. Using the above relations, it is seen that both the amplitude and phase in Eq. (3.39) exhibit singularities as $p_{1} \rightarrow p_{1}^{s p}$,

$$
\begin{aligned}
& \mathcal{A} \sim\left(p_{1}-p_{1}^{s p}\right)^{-1 / 2} \\
& \mathcal{P} \sim \ln \left(p_{1}-p_{1}^{s p}\right) .
\end{aligned}
$$

The other momenta which lead to singularity in the amplitude do not matter as we are only running trajectories with positive momenta and above the barrier height. Notice that the singularity assosciated with the phase is very weak (logarithamic) as compared to the square-root singularity of the amplitude. It is clear that the nonlinear transformation of Eq. (3.34) will remove the singularity in the amplitude. With this transformation the integral can be done via stationary phase approximation which is exact for the parabolic barrier. A little bit of algebra leads to the exact result for the probability

$$
P(E)=\left(1+e^{2 \pi E / \hbar \omega}\right)^{-1} .
$$


In one dimension we were successful in dealing with the singularity as it is possible, a priori, to locate the singularity. The reason being that we are given an invariant function in phase space assosciated with the trajectories which characterizes the separatrix. In one dimensional cases the Hamiltonian (total energy) provides us with such an invariant function. In addition, the singularity was removable as demonstrated in this section. Thus, the numerical integration of the oscillatory integrals presented no particular problem. However, a different kind of problem presents itself in two dimensional collinear scattering systems. Preliminary studies suggest that there are certain singularities which are not removable. These singularities correspond to chaotic scattering[70] in the system. It will be shown in the next chapter that periodic orbit dividing surfaces (PODS)[8] result in these non-removable singularities and some suggestions are offered to efficiently deal with them.

\subsection{Appendix II: Direct semiclassical approach to thermal rate constant $k(T)$-challenges and a new transition state result}

The main focus of this chapter was to formulate a hybrid approach to calculate state-to-state S-matrix elements. Once the S-matrix elements have been calculated, the thermal rate constant $k(T)$ can be obatined via Eq. (3.2). In order to be accurate we would calculate the cummulative reaction probability $N(E)$ over a wide energy range with a fine enough grid in $E$ and then perform a Boltzmann average. However, it would be more efficient if we could directly calculate $k(T)$ without recourse to calculating $N(E)$ first. In recent years remarkable progress has been made towards directly calculating $k(T)$ by exact quantum methods[49, 74]. Again, we are interested in semiclassical methods to compute $k(T)$ for applications to larger systems. Perhaps it is feasible to make use of a reduced dimensionality exact calculation and correct for the neglected, weakly coupled degrees of freedom within a semiclassical approach. At present, we will not go into the details as to how it could be done 


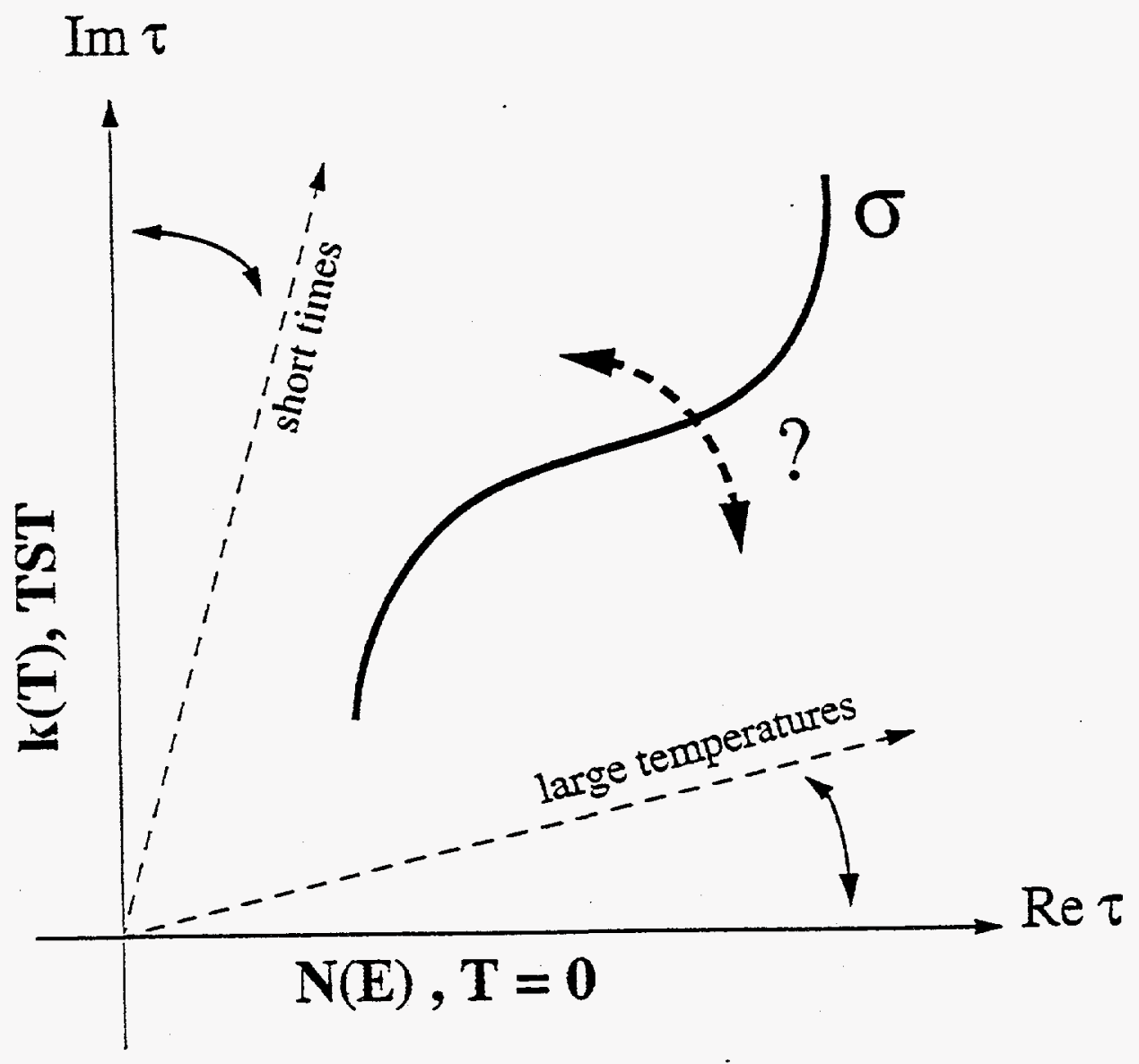

Figure 3.4: This sketch summarizes the difficulty in calculating $k(T)$ semiclassically. $\tau$ denotes complex time and $\sigma$ denotes some Stokes'surface. It is possible to analytically continue the semiclassical propagator from either the real or imaginary time axis as long as the Stokes' surface is not crossed. 
but indicate some problems that prohibit a direct semiclassical approach.

A formally exact expression for the thermal rate constant can be written down as

$$
k(T)=\frac{1}{Q_{r}} \operatorname{Tr}\left[e^{-\beta \hat{H}} \hat{F} \hat{\mathcal{P}}\right],
$$

where $\hat{\mathcal{P}}$ is a projection operator onto reactive space, $Q_{r}$ is the reactant partition function and $\hat{F}$ is a symmetrized flux operator. With the following choice of the projection operator

$$
\hat{\mathcal{P}}=\lim _{t \rightarrow \infty} e^{-i \hat{H} t / \hbar} h(\hat{p}) e^{i \hat{H} t / \hbar},
$$

we can write down the thermal rate constant as

$$
k(T)=\frac{1}{Q_{r}} \lim _{t \rightarrow \infty} \operatorname{Tr}\left[h(\hat{p}) e^{i \hat{H} \tau^{\star} / \hbar} \hat{F} e^{-i \hat{H} \tau / \hbar} h(\hat{p})\right],
$$

where $h(\hat{p})$ is a momentum step function and $\tau$ denotes complex time, $\tau=t-i \beta \hbar / 2$ with $\beta=\left(k_{B} T\right)^{-1}$. A direct calculation of $k(T)$ thus involves the propagator in complex time $\tau$. In order to implement Eq. (3.45) semiclassically, we need to know the analog of the Van Vleck-Gutzwiller approximation for complex time. A first guess is to simply analytically continue the real time result in Eq. (3.13). Unfortunately, this is completely wrong due to the fact that the semiclassical approximation in Eq. (3.13) is an asymptotic result. It is important to realize that asymptotic expressions cannot be analytically continued in a naive fashion since the asymptotic series is valid within a certain region only. In another region, the asymptotic approximation to a function can be of a completely different form. The boundary between these different regions is known as the Stokes' lines or surfaces. On the other hand, if we had an analytic series representing a function then it is possible to analytically continue the series. As a simple example, consider the integral representation of the Airy function

$$
\operatorname{Ai}(z)=\frac{1}{2 \pi} \int_{-\infty}^{\infty} d k \exp \left(i k z+\frac{i k^{3}}{3}\right)
$$

Stationary phase approximation leads to the following asymptotic forms for the Airy function[72]

$$
\begin{array}{lr}
\operatorname{Ai}(z) \approx \frac{1}{2 \pi^{1 / 2} z^{1 / 4}} \exp \left(-\frac{2}{3} z^{3 / 2}\right) & \text { real } z>0 \\
\operatorname{Ai}(z) \approx \frac{1}{\pi^{1 / 2}(-z)^{1 / 4}} \cos \left[\frac{2}{3}(-z)^{3 / 2}-\frac{\pi}{4}\right] & \text { real } z<0 .
\end{array}
$$


It is obvious that the asymptotic form for the Airy function for negative real $z$ cannot be obtained by simply substituting $-z$ in the asymptotic form of $\mathrm{Ai}(z)$ for positive real $z$. This is due to the fact that in case of the Airy function there are three stokes lines in the complex $z$ plane and we cross one of them in going from $z$ to $-z$. It is also clear that on crossing a stokes line, the asymptotic form does not merely change by an overall phase. Asymptotic series actually have a discontinuity at the stokes boundary. There are stokes rules which tell us as to how to continue an asymptotic series across the stokes boundaries. However, the statement of these rules and the mapping of the stokes boundary themselves are extremely difficult for multidimensional systems. In fact a proper generalization of the Van Vleck-Gutzwiller propagator to complex time is a long-standing open problem in semiclassical dynamics. Note that the semiclassical propagator in purely imaginary time is relatively straightforward. This is due to the fact that in classical mechanics purely imaginary time dynamics on a potential $V$ can be mapped over to purely real time dynamics on the upside-down potential $-V$. Figure (3.4) schematically summarizes the difficulty. In the complex time plane, it might be possible to move off the real axis and calculate rates for large temperatures. It is also possible to move off the purely imaginary time axis (TST calculation, real time $t=0$ ) to correct for short time dynamics. But the presence of stokes boundaries $\sigma$ prevents us from rotating too far out of the real or imaginary time axis. Even if we did have the semiclassical propagator for complex times we would have to deal with the issue of complex valued classical trajectories. In one dimension it is possible to keep the trajectory real by appropriate choice of the time contour but it is not possible to do so in a general multidimensional system[76]. These are some of the main difficulties involved in a direct semiclassical calculation for the thermal rate constant. Nevertheless, from a practical viewpoint, it is perhaps better to calculate $k(T)$ in the TST approximation first and then move off the imaginary time axis to correct for real time dynamics. 


\subsubsection{A new semiclassical TST result for $k(T)$}

The starting point is Eq. (3.45) with the real time set to zero. This is an approximation as the projection operator commutes with the Hamiltonian only in the $t \rightarrow \infty$ limit. Subtleties aside, we can write the transition state approximation as

$$
k(T) Q_{r} \approx \operatorname{Tr}\left[e^{-\beta \hat{H}} \hat{F} h(\hat{p})\right] \equiv k_{T S T}(T) Q_{r}
$$

The result is stated in one dimension and is relatively easy to generalize to many degrees of freedom. Inserting a complete set of position states we get

$$
k_{T S T}(T) Q_{r}=\int_{x_{1}, x_{2}}\left\langle x_{1}\left|e^{-\beta \hat{H}}\right| x_{2}\right\rangle\left\langle x_{2}|\hat{F} \hat{\mathcal{P}}| x_{1}\right\rangle
$$

For the imaginary time propagator we perform a semiclassical approximation and use Weyl rules[73] to calculate the flux-projection operator matrix elements. The final result in an initial value representation is

$$
k_{T S T}(T) Q_{r}=\frac{-\hbar}{\pi m} \int_{x_{1}, p_{1}} \mathcal{A}\left(x_{1}, x_{2} ; \tau_{B}\right) \exp \left(\frac{-\mathcal{S}\left(x_{1}, x_{2} ; \tau_{B}\right)}{\hbar}\right)
$$

where $x_{2}=x_{2}\left(x_{1}, p_{1} ; \tau_{B}\right), \tau_{B}=\hbar \beta$ and

$$
\mathcal{A}\left(x_{1}, x_{2} ; \tau_{B}\right) \equiv\left(\frac{1}{2 \pi \hbar} \frac{\partial x_{2}}{\partial p_{1}}\right)^{1 / 2} \frac{\delta\left(x_{1}+x_{2}\right)}{\left(x_{1}-x_{2}\right)^{2}}
$$

and $\mathcal{S}$ is the classical action on the upside-down potential

$$
\mathcal{S}\left(x_{1}, x_{2} ; \tau_{B}\right)=\int_{0}^{\tau_{B}} d \tau[p(\tau) x(\tau)-\mathcal{H}(p(\tau), x(\tau))]
$$

Thus trajectories are evolved on the upside-down potential from time 0 to $\tau_{B}$ and the thermal rate constant in the TST approximation is calculated from Eq. (3.52). It is easily checked that Eq. (3.52) gives the exact results for the free particle and the parabolic barrier cases.

Note that the resulting integrals are not oscillatory anymore. In fact only a finite (perhaps small) number of trajectories contribute to the integral due to the delta function restriciton. It would be interesting to see as to how Eq. (3.52) can now be corrected for real time dynamics i.e., take into account the fact that there can be barrier recrossings. 


\section{Chapter 4}

\section{Application to collinear $A+B C$ reactions}

\subsection{Introduction}

In the previous chapter we described a semiclassical approach to scattering theory in order to calculate state-to-state S-matrix elements. The impressive success and numerical advantages of the method were self-evident from the example of the Eckart barrier. In order for the method to be useful for general multidimensional problems we need to demonstrate similar quantitative and qualitative results for two and three dimensional systems. Collinear i.e., two degrees of freedom reactions have been a fertile test ground for exact quantum as well as approximate methodologies[41, $42,44]$. From a fundamental viewpoint, the classical dynamics of such systems is also considerably rich and interesting[19].

Collinear atom-diatom collisions also offer a representative case to study the effectiveness of the hybrid semiclassical approach. It is now possible, in contrast to one dimensional cases, to systematically study the effect of choosing various approximate trial wavefunctions on the resulting S-matrix. In the previous chapter we applied the method to a one dimensional test case with very stringent choice of parameters and trial wavefunctions. However, a very significant aspect of chemical reaction dynamics i.e., resonances do not manifest themselves in one dimensional barrier problems. It is 
well known that resonances give rise to sharp features for the transition probabilities in collinear systems[41]. Systems with larger than two degrees of freedom also have resonance features, but are typically of less importance. Thus, it becomes important to apply the semiclassical method to collinear reactions in order to completely assess the reliability and accuracy of the approach.

In this chapter we will apply the theoretical ideas developed in the previous section to the collinear $\mathrm{H}+\mathrm{H}_{2}$ reaction. This reaction, apart from being a very good test problem, has been thoroughly studied both classically and via exact quantum methods $[8,44]$. In addition, we have a very reliable $a b$ initio potential energy surface for this system in order to perform classical dynamics. An analysis of the phase space integrals for this reaction reveals a significant bottleneck to the calculations arising from chaotic scattering trajectories[70]. In the next few sections we describe the choice of parameters and trial wavefunctions for the system and offer suggestions to handle the numerical problems due to chaotic scattering.

\subsection{The Jacobi Hamiltonian}

For a general collinear reaction $A+B C$ it is convenient to use Jacobi coordinates $(r, R)$. Here $r$ is the vibrational coordinate of the diatom $B C$ and $R$ is the translational coordinate describing the relative motion of the atom and the diatom. These coordinates are Cartesian for a collinear system. The classical Hamiltonian is given by

$$
\mathcal{H}\left(P_{r}, P_{R}, r, R\right)=\frac{1}{2 m_{r}} P_{r}^{2}+\frac{1}{2 m_{R}} P_{R}^{2}+V(r, R),
$$

where $P_{r}$ and $P_{R}$ are the momenta conjugate to the coordinates $r$ and $R$ respectively. The corresponding reduced masses are

$$
\begin{aligned}
m_{r} & =\frac{m_{B} m_{C}}{m_{B}+m_{C}} \\
m_{R} & =\frac{m_{A}\left(m_{B}+m_{C}\right)}{m_{A}+m_{B}+m_{C}} .
\end{aligned}
$$

Classical trajectories are evolved with this Jacobi Hamiltonian and the potential $V(r, R)$ is the Liu-Siegbahn-Horowitz-Truhlar (LSTH) ab initio potential[75]. Note 
that we have two sets of Jacobi coordinates, one for describing the reactants $A+B C$ and another set for describing the products $A B+C$. Throughout this chapter superscripts $\beta$ and $\alpha$ will be used to indicate the product and reactant Jacobi coordinates respectively. It is important to distinguish the two sets of ccordinates as we are concerned with reactive scattering. The transformation between product and reactant Jacobi coordinates is

$$
\begin{aligned}
r^{\beta} & =R^{\alpha}-\left[\frac{m_{C}}{m_{B}+m_{C}}\right] r^{\alpha} \\
R^{\beta} & =\left[\frac{m_{A}}{m_{A}+m_{B}}\right] R^{\alpha}+\left[\frac{m_{A} m_{B}}{m_{R}\left(m_{A}+m_{B}\right)}\right] r^{\alpha}
\end{aligned}
$$

with the corresponding transformation for the conjugate momenta $\left(P_{r}^{\beta}, P_{R}^{\beta}\right)$.

\subsection{The semiclassical S-matrix}

As shown in the previous chapter, the semiclassical correction term to the KVP S-matrix can be written down as the four dimensional phase space integral

$$
\begin{aligned}
\Delta S_{n_{\beta}, n_{\alpha}}(E) & =\frac{1}{2 \pi i \hbar^{3}} \\
& \times \int_{\mathbf{x}_{1}^{\alpha}, \mathbf{P}_{1}^{\alpha}, t} \mathcal{A}_{n_{\beta}, n_{\alpha}}\left(\mathbf{x}_{2}^{\beta}, \mathbf{x}_{1}^{\alpha} ; t\right) \exp \left(\frac{i \mathcal{P}\left(\mathbf{x}_{2}^{\beta}, \mathbf{x}_{1}^{\alpha} ; t\right)}{\hbar}\right),
\end{aligned}
$$

where we have explicitly indicated the reactant and product channels and

$$
\begin{aligned}
\mathcal{A}_{n_{\beta}, n_{\alpha}}\left(\mathbf{x}_{2}^{\beta}, \mathbf{x}_{1}^{\alpha} ; t\right) & \equiv\left|\operatorname{Det}\left(\frac{\partial \mathbf{x}_{2}^{\beta}}{\partial \mathbf{p}_{1}^{\alpha}}\right)\right|^{1 / 2} \varphi_{n_{\beta}}\left(\mathbf{x}_{2}^{\beta}\right) \varphi_{n_{\alpha}}\left(\mathbf{x}_{1}^{\alpha}\right) \\
\mathcal{P}\left(\mathbf{x}_{2}^{\beta}, \mathbf{x}_{1}^{\alpha} ; t\right) & \equiv \mathcal{S}\left(\mathbf{x}_{2}^{\beta}, \mathbf{x}_{1}^{\alpha} ; t\right)+E t-\frac{\pi \hbar \mu}{2} \\
\mathbf{x}_{2}^{\beta} & \equiv \mathbf{x}_{2}^{\beta}\left(\mathbf{x}_{1}^{\alpha}, \mathbf{p}_{1}^{\alpha} ; t\right)
\end{aligned}
$$

In the above expression we have denoted $\mathbf{x} \equiv(r, R)$ and $\mathbf{p} \equiv\left(P_{r}, P_{R}\right)$ for compactness. However, we want to work with an equivalent expression which does not involve the time integral and the integral over translational coordinate $R$. In order to go to such an expression, we run classical trajectories from a fixed asymptotic value of the initial translational Jacobi coordinate to a fixed asymptotic value of the final translational 
Jacobi coordinate. In other words, classical trajectories evolve from $R_{1}^{\alpha}=\bar{R}$ to $R_{2}^{\beta}=\bar{R}$. The time integral is trivially done as for a given set of initial conditions $\left(r_{1}^{\alpha}, \bar{R}, P_{r 1}^{\alpha}, P_{R 1}^{\alpha}\right)$ the time $\bar{t}$ is fixed by the relation $R_{2}^{\beta}\left(r_{1}^{\alpha}, \bar{R}, P_{r 1}^{\alpha}, P_{R 1}^{\alpha} ; \bar{t}\right)=\bar{R}$. As a result, the full S-matrix is given by[76]

$$
\begin{aligned}
S_{n_{\beta}, n_{\alpha}}(E) & =-\frac{1}{2 \pi i \hbar} \\
& \times \int_{\tau_{1}^{\alpha}, P_{r 1}^{\alpha}, P_{R 1}^{\alpha}} \mathcal{A}_{n_{\beta}, n_{\alpha}}\left(r_{2}^{\beta}, r_{1}^{\alpha} ; \bar{t}, \bar{R}\right) \exp \left(\frac{i \mathcal{P}\left(r_{2}^{\beta}, r_{1}^{\alpha} ; \bar{t}, \bar{R}\right)}{\hbar}\right),
\end{aligned}
$$

where

$$
\begin{aligned}
\mathcal{A}_{n_{\beta}, n_{\alpha}}\left(r_{2}^{\beta}, r_{1}^{\alpha} ; \bar{t}, \bar{R}\right) & \equiv \frac{m_{R}}{\left|P_{R 2}^{\beta}\right|}\left|\operatorname{Det}\left(\frac{\partial \mathbf{x}_{2}^{\beta}}{\partial \mathbf{p}_{1}^{\alpha}}\right)_{\bar{R}}\right|^{1 / 2} \varphi_{n_{\beta}}\left(r_{2}^{\beta} ; \bar{R}\right) \varphi_{n_{\alpha}}\left(r_{1}^{\alpha} ; \bar{R}\right) \\
\mathcal{P}\left(r_{2}^{\beta}, r_{1}^{\alpha} ; \bar{t}, \bar{R}\right) & \equiv \mathcal{S}\left(r_{1}^{\alpha}, \mathbf{p}_{1}^{\alpha} ; \bar{t}, \bar{R}\right)+E \bar{t}-\frac{\pi \hbar \mu}{2} \\
r_{2}^{\beta} & \equiv r_{2}^{\beta}\left(r_{1}^{\alpha}, \mathbf{p}_{1}^{\alpha} ; \bar{t}, \bar{R}\right)
\end{aligned}
$$

and we have explicitly denoted the parametric dependence of the relevant functions on $\bar{R}$ and $\bar{t}$. Note that even though the Jacobian determinant is needed only at a time $\bar{t}$, we still require it along the trajectory in order to keep track of the Maslov index $[60] \mu$.

\subsubsection{Choice of the trial wavefunctions}

In Eq. (4.7) we have to specify the form of the trial wavefunctions $\varphi$. For the present case of collinear $\mathrm{H}+\mathrm{H}_{2}$ we take the vibrational part to be eigenfunctions of an appropriate Morse oscillator[77] potential $V(r)$

$$
V(r)=D\left[1-\exp \left(-a\left(r-r_{0}\right)\right)\right]^{2}
$$

and plane waves for the translational degree of freedom. The parameters for the Morse potential $D, a, r_{0}$ are chosen to agree with the LSTH potential. Thus we have

$$
\varphi_{n}(r ; \bar{R})=v_{n}^{1 / 2} e^{-i k_{n} \bar{R}} \psi_{n}(r)
$$

where $v_{n}$ is the asymptotic velocity in channel $n$ and $\psi_{n}$ are Morse oscillator eigenfunctions which can be analytically obtained by solving the corresponding Schrödinger 
equation[77]. The resulting expression is

$$
\psi_{n}(r)=\left[\frac{a \kappa n !}{\Gamma(\kappa+n+1)}\right]^{1 / 2} e^{-z / 2} z^{\kappa / 2} L_{n}^{\kappa}(z)
$$

where $\kappa \equiv \zeta-2 n-1, \Gamma(b)$ is the Gamma function[78] and $L_{n}^{\kappa}(z)$ are the assosciated Laguerre functions[78] evaluated using the following series

$$
\begin{aligned}
\mathrm{L}_{n}^{\kappa}(z) & =\sum_{m=0}^{n} \frac{(-1)^{m}(\kappa+n) !}{m !(n-m) !(\kappa+m) !} z^{m} \\
z & =\zeta \exp \left(-a\left(r-r_{0}\right)\right) ; \quad \zeta=\frac{2}{a \hbar} \sqrt{2 m_{r} D}
\end{aligned}
$$

For collinear $H+H_{2}$ the parameters were chosen as $D=0.17447 a u, r_{0}=1.402 a u$ and $a=1.026 \mathrm{au}$. The parameters $D$ and $r_{0}$ correspond to the depth of the potential and equilibrium bond length of the diatom respectively[75]. The factorials in Eq. (4.11) are evaluated using the Gamma function definition $\Gamma(b)=(b-1)$ ! since $\kappa$ is not an integer. The asymptotic velocity in channel $n$ is given by

$$
\begin{aligned}
& v_{n}=\sqrt{\frac{2\left(E-\varepsilon_{n}\right)}{m_{R}}} \\
& \varepsilon_{n}=-D\left[\left(\frac{2 n+1}{\zeta}\right)^{2}-2\left(\frac{2 n+1}{\zeta}\right)\right]
\end{aligned}
$$

where $\varepsilon_{n}$ are the eigenenergies of the Morse oscillator. Note that we have again chosen a very simple form for our trial wavefunctions in order to test the semiclassical model in the extreme limits.

\subsubsection{Evaluating the Jacobian determinant}

The Jacobian determinant in Eq. (4.7) is part of the full monodromy matrix and we require the determinant along each classical trajectory in order to properly account for the Maslov index. As discussed in the previous chapter, the time evolution of the Jacobian requires the knowledge of second derivatives of the potential with respect to the Jacobi coordinates. Unfortunately, the LSTH ab initio potential provides us with only the first derivatives of the potential. It is possible to use the 


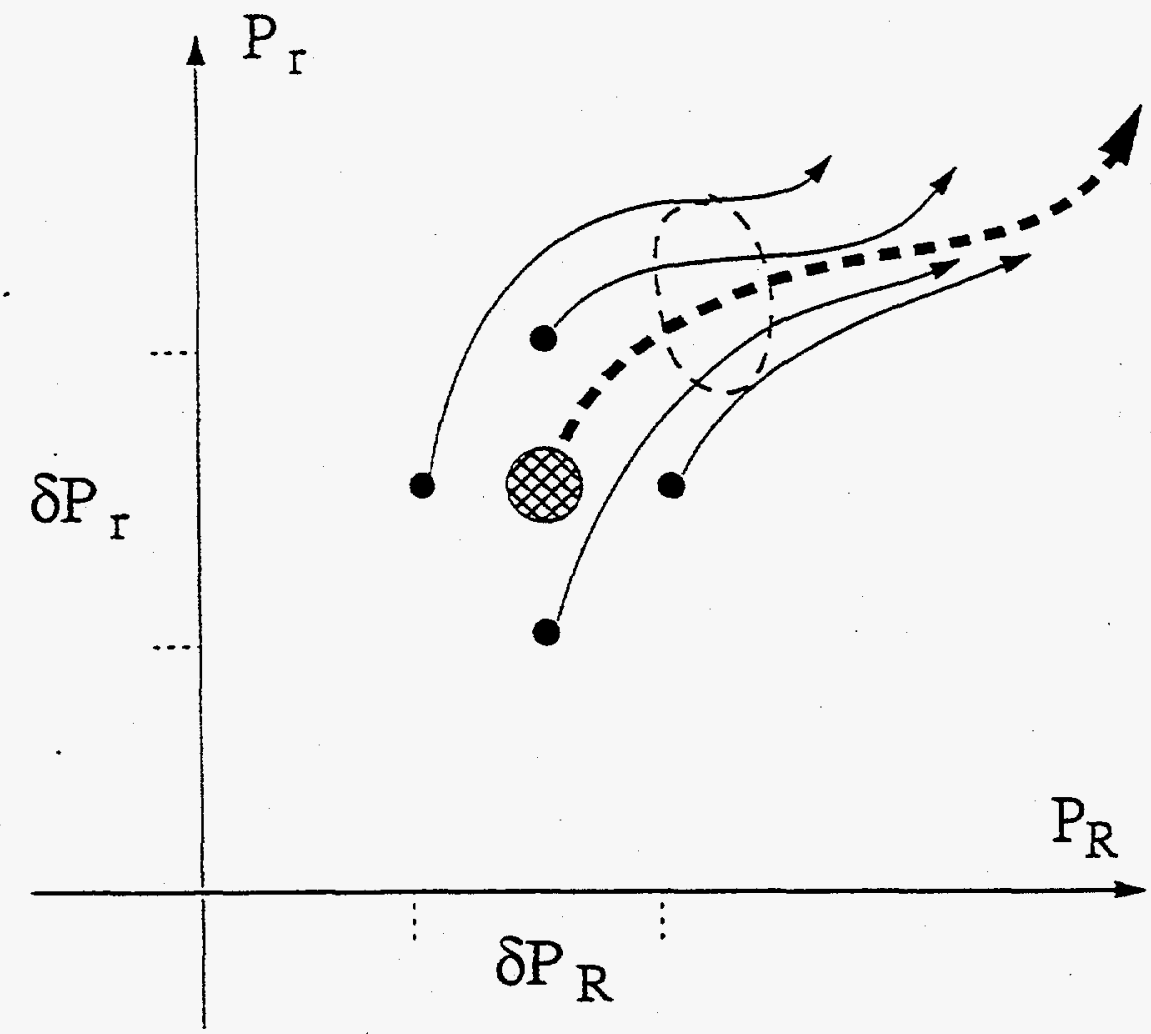

Figure 4.1: This figure summarizes the startegy outlined in this chapter in order to determine the Jacobian determinant along a classical trajectory. For each trajectory, sketched by the heary dotted line, a set of four auxillary trajectories are evolved with the same initial positions $\left(r_{1}^{\alpha}, \bar{R}\right)$ but different momenta. These aurillary trajectories are schematically shown as thin solid lines. This trajectory bundle is then used to determine the Jacobian at time $t$.

first derivative information to obtain the necessary second derivatives by finite difference methods but, we adopt a different strategy to evaluate the Jacobian which is very easy to implement in problems with very high degrees of freedom. The firite difference approach using first derivatives of the potential can be very expensive and generally unreliable for larger systems.

Figure (4.1) summarizes the startegy. We evolve four aurillary trajectories simultaneously with our original trajectory which differ in their inital momenta but with the same initial positions $\left(r_{1}^{\alpha}, \bar{R}\right)$. The initial momenta for the four aurillary trajectories are $\left(P_{r}^{\alpha} \pm \delta P_{r}^{\alpha} / 2, P_{R}^{\alpha} \pm \delta P_{R}^{\alpha} / 2\right)$ with the original trajectory $\left(P_{r}^{\alpha}, P_{R}^{\alpha}\right)$. This trajectory bundle will give us the Jacobian determinant at any time $t$ along the 
trajectory. For example, one of the elements of the Jacobian is obtained as[78]

$$
\frac{\partial r_{2}^{\beta}}{\partial P_{R 1}}(t) \approx \frac{1}{\delta P_{R 1}}\left[r_{2}^{\beta}\left(t ; P_{R 1}^{\alpha}+\frac{\delta P_{R 1}^{\alpha}}{2}\right)-r_{2}^{\beta}\left(t ; P_{R 1}^{\alpha}-\frac{\delta P_{R 1}^{\alpha}}{2}\right)\right],
$$

and similarly for the other three elements. In the above expression we have supressed the dependence of $r_{2}^{\beta}$ on the other initial conditions for sake of clarity. For our case we found that a spacing of about $10^{-8}$ for the trajectory bundle is optimal. If the spacing is too small it leads to numerical errors due to the limits on the precision with which we are integrating the trajectories. Applying this method to the one dimensional Eckart barrier yielded results which were in very good agreement with the results obtained in the previous chapter.

\subsection{Behaviour of the integrand}

We are now ready to perform the integrals in Eq. (4.6). However, this is not an easy task at all due to the complicated nature of the integrand. Figure (4.2) shows a typical plot of the imaginary part of the integrand as a function of the initial translational momentum with the other variables fixed. The nonreactive trajectories for the system are identified and assigned zero amplitudes. It is clear from the figure that there are regions of reactive trajectories and nonreactive trajectories, both having a very smooth behaviour as a function of $P_{R}^{\alpha}$. More crucially, it is seen that the region seperating the reactive and nonreactive trajectories are extremely complicated. In particular, these boundaries between reactive and nonreactive trajectories manifest themselves as singularities in the integrand. These singularities occur at energies much higher than the barrier height energy of $0.425 \mathrm{eV}$. It is important to note that the results in figure (4.2) do not change when we do the trajectory calculations in an more accurate fashion.

In fact the situation is much more complex than that suggested by figure (4.2). Looking at the integrand more closely around one of these boundaries shows that these singularities arise due to irregular or chaotic scattering trajectories. Figure (4.3) shows the integrand in greater detail around one of the boundaries. Interspersed in that random smatter of points are both reactive and nonreactive trajectories. This 


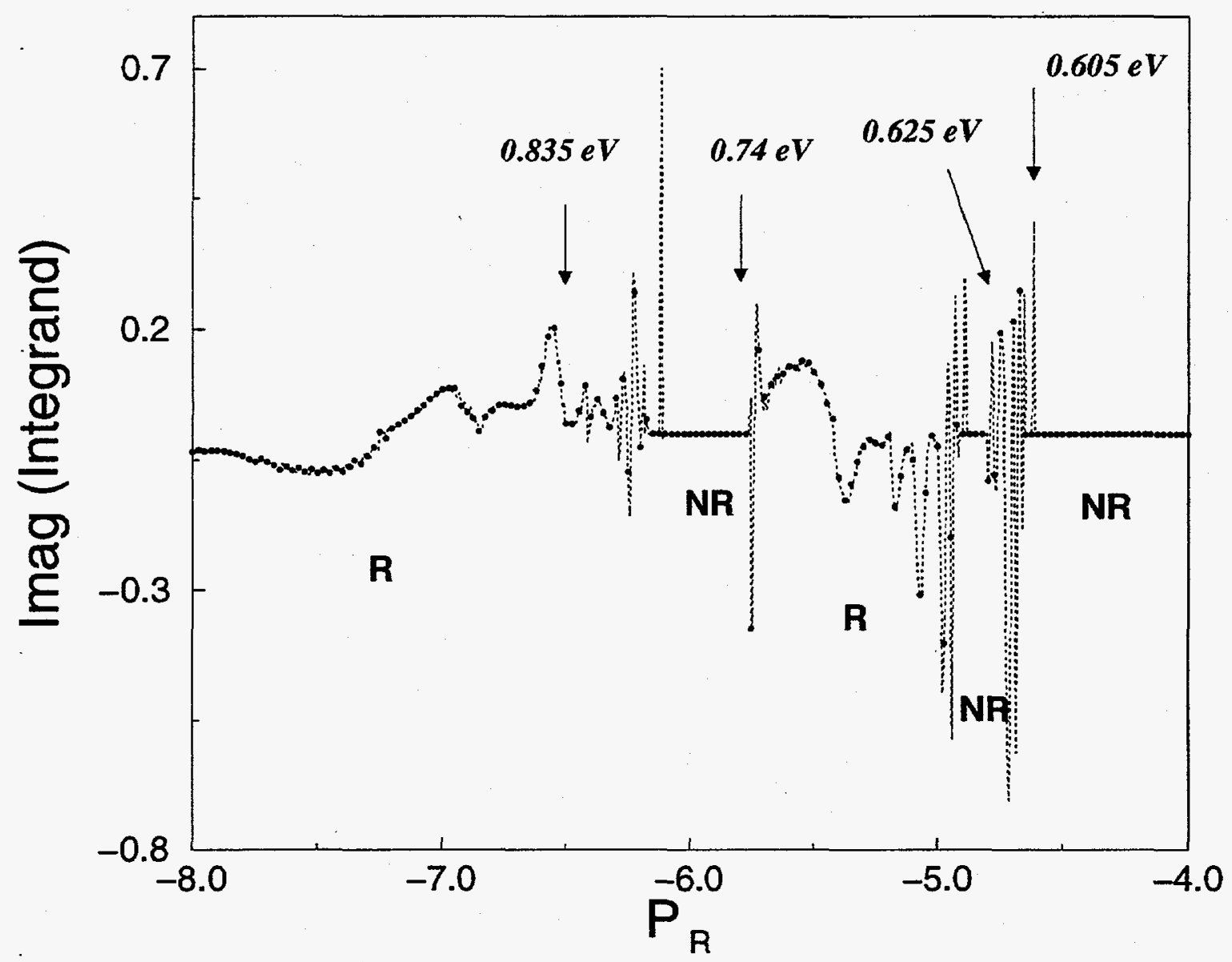

Figure 4.2: Shown here is a typical plot of the imaginary part of the integrand as a function of $P_{R}^{\alpha}$ with the other variables being held fixed. The labels $R$ and NR denote reactive and nonreactive trajectories respectively. The solid points are calculations done with a $P_{R}^{\alpha}$ grid much coarser than the results shown by the dotted line. Notice the singularities at the boundaries between $\mathrm{R}$ and $\mathrm{NR}$ trajectories. The contribution from $\mathrm{R}$ trajectories are very smooth. Some of the total energies of the trajectories are also indicated in the figure. For comparison, the barrier height for collinear $\mathrm{H}+\mathrm{H}_{2}$ reaction is about $0.425 \mathrm{eV}$. 


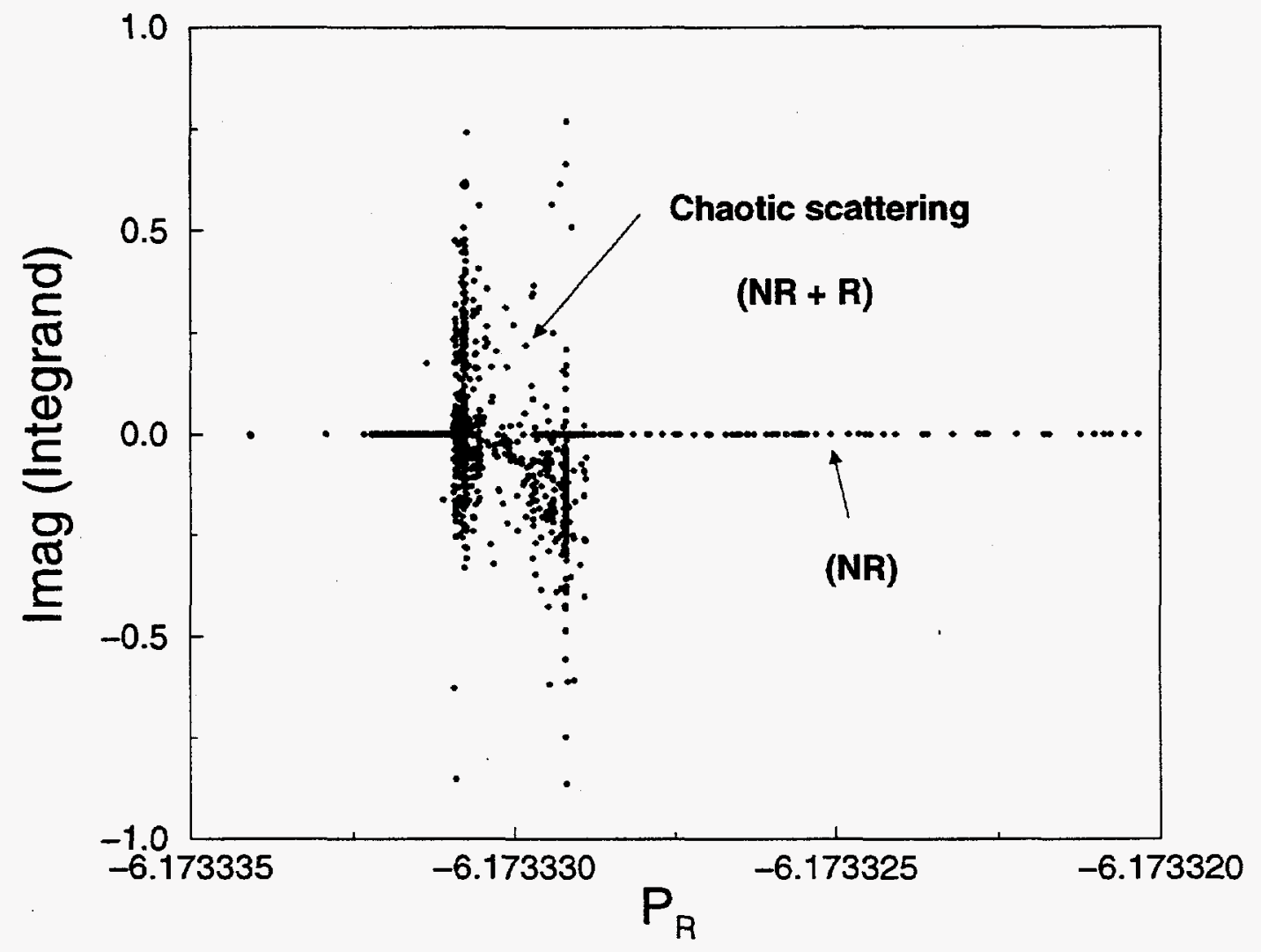

Figure 4.3: This plot shows the imaginary part of the integrand as a function of $P_{R}^{\alpha}$ near a R-NR boundary. It is clear that the singularity is due to chaotic scattering and hence not integrable.

shows the sensitivity of the trajectories to initial conditions. It is obvious for well known reasons that these singularities are nonintegrable[70]. Thus, any naive numerical integration scheme has negligible chances of converging.

The phenomenon of chaotic scattering is well known in two dimensional dynamical systems[70, 81]. Considerable work has been done to understand chaotic scattering in collinear atom-diatom collisons. It was demonstrated by Pechukas, Pollak, Davis and many others $[8,80]$ that these boundaries are actually periodic orbit dividing surfaces (PODS). Davis[8, 79] has done a thorough analysis of classical dynamics around these PODS by explicitly mapping them out. It is now well known that there can be a total of nine such PODS for the collinear $H+H_{2}$ reaction[79]. Furthermore, the existence of more than one PODS was linked to the failure of variational transition state theory[80]. In figure (4.4) we show three different kinds of 
reactive trajectories-a simple reactive trajectory which crosses the transition state once, a reactive trajectory which spends a lot of time near the asymmetric stretch periodic orbit and the most complicated one at an energy of about $0.75 \mathrm{eV}$ which recrosses the transition state. The latter kind of trajectory are the ones which exist near the boundaries. For the moment, let us think of the PODS as some kind of adiabatic barriers. Then the separatrix assosciated with them have an extremely complicated structure as compared to an one dimensional barrier like the Eckart barrier. Thus, it is not possible to transform away these singularities in a manner analogous to what we did for the Eckart barrier ${ }^{1}$.

Given this complicated situation, it is natural to ask as to how is it possible to make any progress with integrating Eq. (4.7). An obvious approach is to explicitly map out all the periodic orbits and use a customized integration scheme in order to perform the integrals. This is certainly feasible in two dimensions but impractical in higher dimensions. The mapping of periodic orbits in three or higher degrees of freedom can become prohibitively difficult. Since we are interested in applying the semiclassical hybrid approach to multidimensional systems we have to develop a method which does not rely upon a priori knowledge of the PODS. This, however, is a very difficult task and still an open question in numerical analysis. Nevetheless, as a beginning effort, the modified Filinov method[82] may have some advantages in dealing with this problem.

\subsubsection{The modified Filinov method}

Consider a generic multidimensional integral

$$
\mathcal{I}=\int_{\mathbf{p}} \mathcal{A}(\mathbf{p}) e^{i \mathcal{P}(\mathbf{p})}
$$

Following Filinov, we insert unity in the form

$$
1=\int_{\mathbf{p}_{0}}\left[\operatorname{Det}\left(\frac{\mathbf{B}}{2 \pi}\right)\right]^{1 / 2} \exp \left(-\frac{1}{2}\left(\mathbf{p}-\mathbf{p}_{0}\right) \cdot \mathbf{B} \cdot\left(\mathbf{p}-\mathbf{p}_{0}\right),\right.
$$

\footnotetext{
${ }^{1}$ Please refer to appendix I of chapter 2.
} 


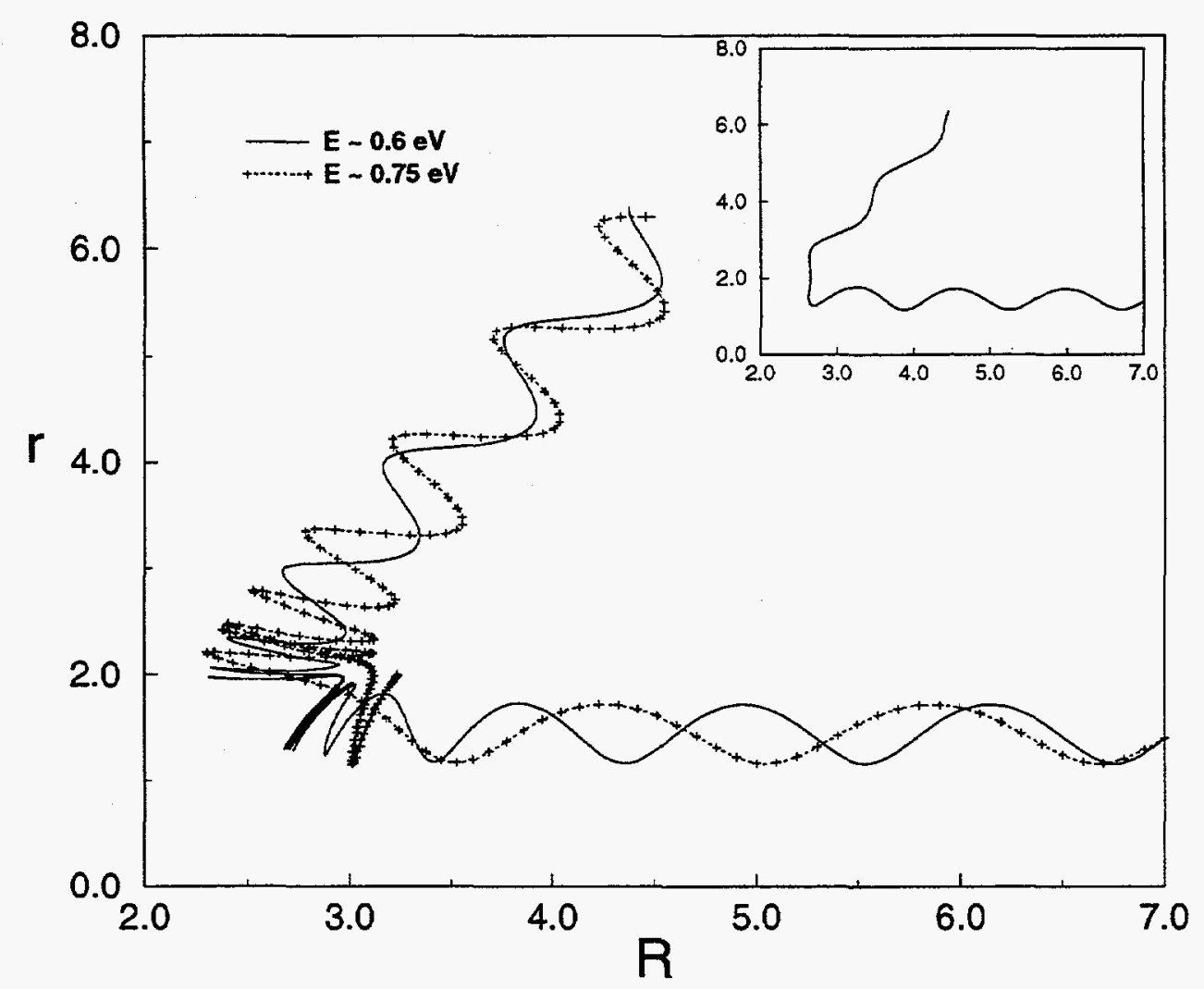

Figure 4.4: Three different kinds of reactive trajectories are shown in this figure. The inset shows a trajectory with $E \approx 0.6 \mathrm{eV}$ which crosses the transition state only once. In the main figure, the trajectory with $E \approx 0.6 \mathrm{eV}$ also crosses the transition state only once but spends considerable time trapped near the asymmetric stretch periodic orbit. Finally, the trajectory with $E \approx 0.75 \mathrm{eV}$ violates transition state assumption as it recrosses the transition state and spends some time trapped near two of the PODS. 
where $B$ is a positive definite matrix, into the integrand of Eq. (4.14). Interchanging the order of integration, the integral becomes

$$
\mathcal{I}=\int_{\mathbf{p}^{0}}\left[\operatorname{Det}\left(\frac{\mathbf{B}}{2 \pi}\right)\right]^{1 / 2} \int_{\mathbf{p}} \mathcal{A}(\mathbf{p}) \exp \left(i \mathcal{P}(\mathbf{p})-\frac{1}{2}\left(\mathbf{p}-\mathbf{p}_{0}\right) \cdot \mathbf{B} \cdot\left(\mathbf{p}-\mathbf{p}_{0}\right) .\right.
$$

Note that in the above expression the gaussian factor insures that the integral is dominated by values of $\mathbf{p} \approx \mathbf{p}_{0}$. Thus, we expand $\mathcal{P}(\mathbf{p})$ in a Taylor series about $\mathbf{p}_{0}$ through quadratic terms:

$$
\mathcal{P}(\mathbf{p}) \approx \mathcal{P}\left(\mathbf{p}_{0}\right)+\mathcal{P}_{1}\left(\mathbf{p}_{0}\right) \cdot\left(\mathbf{p}-\mathbf{p}_{0}\right)+\frac{1}{2}\left(\mathbf{p}-\mathbf{p}_{0}\right) \cdot \mathcal{P}_{2}\left(\mathbf{p}_{0}\right) \cdot\left(\mathbf{p}-\mathbf{p}_{0}\right)
$$

where $\mathcal{P}_{1}\left(\mathbf{p}_{0}\right) \equiv \partial \mathcal{P} / \partial \mathbf{p}$ and $\mathcal{P}_{2}\left(\mathbf{p}_{0}\right) \equiv \partial^{2} \mathcal{P} / \partial \mathbf{p} \partial \mathbf{p}$. With this quadratic expansion we can analytically integrate over $\mathbf{p}$, obtaining

$$
\begin{aligned}
\mathcal{I} & \approx \mathcal{I}(\mathbf{B}) \\
& \equiv \int_{\mathbf{p}_{0}}\left[\operatorname{Det}\left(\mathbf{1}-i \mathcal{P}_{2}\left(\mathbf{p}_{0}\right) \cdot \mathbf{B}^{-1}\right)\right]^{-1 / 2} \mathcal{A}\left(\mathbf{p}_{0}\right) \\
& \times \exp \left(i \mathcal{P}\left(\mathbf{p}_{0}\right)-\frac{1}{2} \mathcal{P}_{1}\left(\mathbf{p}_{0}\right) \cdot\left[\mathbf{B}-i \mathcal{P}_{2}\left(\mathbf{p}_{0}\right)\right]^{-1} \cdot \mathcal{P}_{1}\left(\mathbf{p}_{0}\right)\right)
\end{aligned}
$$

The above equation results from the standard Filinov procedure. Note that we have replaced the amplitude $\mathcal{A}(\mathbf{p})$ by the zeroth order term $\mathcal{A}\left(\mathbf{p}_{0}\right)$. It is possible to make a better approximation by including higher order terms but for now we make the simplest possible choice for the amplitude. At this juncture we make a small modification as suggested by Makri and Miller[82] in order to make the approximation more useful. Notice that Eq. (4.15) is true even if $B$ is complex and approximately true if $\mathbf{B}$ is a function of $p_{0}$. Accordingly, we modify $\mathbf{B}$ as

$$
\mathbf{B}=\mathbf{B}\left(\mathbf{p}_{0}\right)=i \mathcal{P}_{2}\left(\mathbf{p}_{0}\right)+\mathbf{b}
$$

as a result of which Eq. (4.18) becomes

$$
\begin{aligned}
\mathcal{I} & \approx \mathcal{I}(\mathbf{b}) \\
& \equiv \int_{\mathbf{p}_{0}}\left[\operatorname{Det}\left(\mathbf{1}+i \mathcal{P}_{2}\left(\mathbf{p}_{0}\right) \cdot \mathbf{b}^{-1}\right)\right]^{1 / 2} \mathcal{A}\left(\mathbf{p}_{0}\right) \\
& \times \exp \left(i \mathcal{P}\left(\mathbf{p}_{0}\right)-\frac{1}{2} \mathcal{P}_{1}\left(\mathbf{p}_{0}\right) \cdot \mathbf{b}^{-1} \cdot \mathcal{P}_{1}\left(\mathbf{p}_{0}\right)\right)
\end{aligned}
$$


where the matrix $\mathbf{b}$ is a constant positive definite matrix. Eq. (4.20) is the modified Filinov result. As a simple choice for the matrix $\mathbf{b}$ we take $\mathbf{b}=b \mathbf{1}$ with $b>0$. One of the main attractive features of Eq. (4.20) is that it has the stationary phase result as its worst limit. It can be shown that

$$
\begin{aligned}
\lim _{b \rightarrow \infty} \mathcal{I}(\mathbf{b}) & =\mathcal{I}_{\text {exact }} \\
\lim _{b \rightarrow 0} \mathcal{I}(\mathbf{b}) & =\mathcal{I}_{\text {sp }},
\end{aligned}
$$

where $\mathcal{I}_{\text {exact }}$ is the exact integral and $\mathcal{I}_{s p}$ is the stationary phase approximation to the exact integral. Thus, it is possible to systematically go beyond stationary phase by increasing the value of $b$. In additon, if $\mathcal{P}(\mathbf{p})$ is a quadratic function then $\mathcal{I}(\mathbf{b})=\mathcal{I}$ for all values of $b$. There are other numerically attractive features of this method[83] but for the purposes of this chapter we will take Eq. (4.20) as the basic result.

\subsubsection{Eckart barrier revisited}

Before applying the modified Filinov trick to the collinear reaction we test it on the one dimensional Eckart barrier problem. Specifically, we want to see the accuracy of the results as compared to the results obtained in the previous chapter. Since in the collinear problem we do not have a priori knowledge of the PODS, we will analogously perform the modified Filinov trick on the Eckart case without integrating out the singularity ${ }^{2}$.

The full S-matrix can be written down in analogy with Eq. (4.6) as ${ }^{3}$

$$
S_{\bar{n}, n}^{s c}(E)=-\frac{v}{(2 \pi i \hbar)^{1 / 2}} \int_{p_{1}} \mathcal{A}\left(p_{1} ; \bar{t}, \bar{x}\right) \exp \left(i \mathcal{P}\left(p_{1} ; \bar{t}, \bar{x}\right)\right)
$$

where we have used a slightly different notation for $\mathcal{A}$ and $\mathcal{P}$ as compared to the ones used in the previous chapter with

$$
\begin{aligned}
& \mathcal{A}\left(p_{1} ; \bar{t}, \bar{x}\right) \equiv \frac{m}{\left|p_{2}\right|}\left|\frac{\partial x_{2}}{\partial p_{1}}\right|_{\bar{x}}^{1 / 2} \\
& \mathcal{P}\left(p_{1} ; \bar{t}, \bar{x}\right) \equiv-2 k \bar{x}+\frac{1}{\hbar}\left[E \bar{t}+\mathcal{S}\left(p_{1} ; \bar{t}, \bar{x}\right)\right]
\end{aligned}
$$

\footnotetext{
${ }^{2}$ In the previous chapter we had explicitly changed variables in order to transform away the singularity in the integrand assosciated with the seperatrix trajectory. Please refer to chapter 2 of this thesis.

${ }^{3}$ See appendix I of the previous chapter.
} 
In order to calculate the Filinov derivatives of the phase $\mathcal{P}$ we have to be careful as the derivatives have to be taken with the condition that $x_{2}=\bar{x}$ is fixed and $\bar{t}=\bar{t}\left(\bar{x}, p_{1}\right)$. Thus, for example the first derivative of the phase with respect to $p_{1}$ is calculated as follows:

$$
\left.\mathcal{P}_{1}\left(p_{1} ; \bar{t}, \bar{x}\right) \equiv \frac{\partial \mathcal{P}}{\partial p_{1}}\right|_{\bar{x}}=\left.\frac{\partial \mathcal{P}}{\partial p_{1}}\right|_{\bar{t}}+\frac{\partial \mathcal{P}}{\partial \bar{t}} \frac{\partial \bar{t}}{\partial p_{1}},
$$

where a subscript denotes the quantity being held fixed. Using the relations

$$
\begin{aligned}
\frac{\partial \mathcal{S}}{\partial p_{1}} & =p_{2} \frac{\partial x_{2}}{\partial p_{1}} \\
\frac{\partial \mathcal{S}}{\partial \bar{t}} & =\mathcal{H} \\
\frac{\partial \bar{t}}{\partial p_{1}} & =-\left.\frac{m}{p_{2}} \frac{\partial x_{2}}{\partial p_{1}}\right|_{\bar{t}}
\end{aligned}
$$

with $\mathcal{H}$ being the classical Hamiltonian, we obtain

$$
\hbar \mathcal{P}_{1}\left(p_{1} ; \bar{t}, \bar{x}\right)=\left.p_{2} \frac{\partial x_{2}}{\partial p_{1}}\right|_{\bar{t}}-\left.\frac{m}{p_{2}}(E+\mathcal{H}) \frac{\partial x_{2}}{\partial p_{1}}\right|_{\bar{t}} .
$$

Similar analysis can be done for the second derivative of the phase obtaining

$$
\begin{aligned}
\hbar \mathcal{P}_{2}\left(p_{1} ; \bar{t}, \bar{x}\right) & =\left\{\left(\frac{2 m}{p_{2}^{2}}(E+\mathcal{H}) \frac{\partial p_{2}}{\partial p_{1}}-\frac{p_{1}}{p_{2}}\right) \frac{\partial x_{2}}{\partial p_{1}}\right. \\
& \left.+\frac{m}{p_{2}} \frac{\partial V}{\partial x}\left(\frac{\partial x_{2}}{\partial p_{1}}\right)^{2}\left[1+\frac{m}{p_{2}^{2}}(E+\mathcal{H})\right]\right\}\left.\right|_{\bar{t}}
\end{aligned}
$$

Thus, the modified Filinow approximation to Eq. (4.22) is given by

$$
\begin{aligned}
S_{\bar{n}, n}^{s c}(E) & \approx S_{\bar{n}, n}^{s c}(E ; b) \\
& =-\frac{v}{(2 \pi i \hbar)^{1 / 2}} \int_{p} \mathcal{A}^{M F}(p ; \bar{t}, \bar{x}) \exp \left(i \mathcal{P}^{M F}(p ; \bar{t}, \bar{x})\right)
\end{aligned}
$$

where

$$
\begin{aligned}
\mathcal{A}^{M F}(p ; \bar{t}, \bar{x}) & \equiv \mathcal{A}(p ; \bar{t}, \bar{x})\left(1+\frac{i \mathcal{P}_{2}(p ; \bar{t}, \bar{x})}{b}\right)^{1 / 2} \\
\mathcal{P}^{M F}(p ; \bar{t}, \bar{x}) & \equiv \mathcal{P}(p ; \bar{t}, \bar{x})+\frac{i}{2 b} \mathcal{P}_{1}^{2}(p ; \bar{t}, \bar{x})
\end{aligned}
$$

Trajectories are now propagated with initial conditions $(p,-\bar{x})$ until a fixed final position $\bar{x}$ is reached and the integral in Eq. (4.28) is calculated. Note that all 


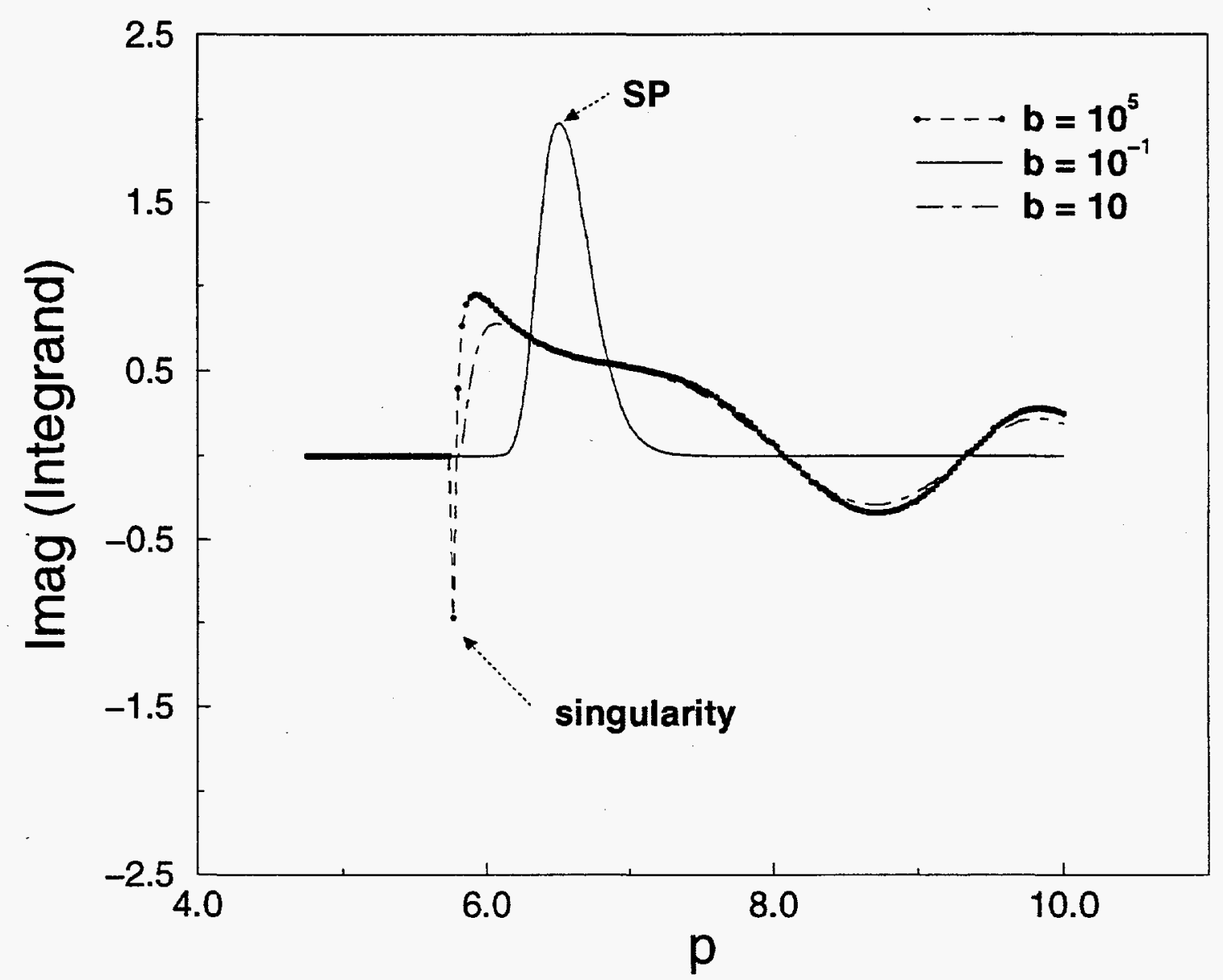

Figure 4.5: This figure shows the imaginary part of the integrand in Eq. (4.28) for various values of the Filinov parameter $b$ for $E=0.55 \mathrm{eV}$. Notice the smooth form of the integrand for very small values of $b$ (stationary phase limit) and the separatrix singularity emerging for larger values of $b$ (exact limit). The stationary phase (SP) and singularity momenta are also indicated on the plot. 
the relevant quantities required for the modified Filinov method are already being computed along the trajectory.

Figure (4.5) shows the imaginary part of the integrand in Eq. (4.28) for various values of the Filinov parameter $b$. The energy $E$ is set equal to $0.55 \mathrm{eV}$. For very small values of $b$ we see that the gaussian factor in the integrand of Eq. (4.28) samples a very narrow region around the stationary phase region and damps out the rest leading to a very smooth behaviour of the integrand. The separatrix singularity is damped out at this value of $b$ and a slightly larger value of $b$ as well. For very large values of $b$ we see the singularity manifest itself in the integrand since we are approaching the exact integral in this limit. It is clear that for $E$ less than or close to the barrier height, the results with a very small value of $b$ will be essentially zero. This is due to the fact that there are no real stationary phase points at these energies for the integrand. It is thus necessary to consider larger values of $b$ in order to obtain the probabilities at these energies. Thus it is seen that a calculation with very small $b$ yields fairly good results for the transmission probabilities at energies above the barrier height. As the value of $b$ increases the tunneling contribution become finite and begin to move towards the correct results. The results are very encouraging and provide some hope that the collinear problem can be dealt with more efficiently in the future.

\subsubsection{Modified Filinov approach to collinear systems}

The modified Filinov method is applied to Eq. (4.6) only to the translational momentum part of the integrand. In general, we could apply it to both the translational and vibrational momentum part of the integrand but we consider the simpler case in this section. The first and second derivatives of the phase are calculated as outlined in the previous section. Again, the derivatives are calculated for fixed value of the translational Jacobi coordinate $\bar{R}$. We will just state the results:

$$
\left.\hbar \mathcal{P}_{1}\right|_{\bar{R}}=\left.\left\{\left[P_{R 2}^{\beta}-\frac{m_{R}}{P_{R 2}^{\beta}}(E+\mathcal{H})\right] \mathcal{M}_{T}+P_{r 2}^{\beta} \mathcal{M}_{V}\right\}\right|_{\bar{t}}
$$




$$
\left.\hbar \mathcal{P}_{2}\right|_{\bar{R}}=\left.\mathcal{P}_{2}^{(1)}\right|_{\bar{t}}+\left.\mathcal{P}_{2}^{(2)}\right|_{\bar{t}},
$$

where

$$
\begin{aligned}
\mathcal{P}_{2}^{(1)} & \approx\left[1+\frac{m_{R}}{P_{R 2}^{\beta} P_{R 2}^{\beta}}(E+\mathcal{H})\right] \mathcal{M}_{T P} \mathcal{M}_{T}-\frac{P_{R 1}^{\alpha}}{P_{R 2}^{\beta}} \mathcal{M}_{T}+\mathcal{M}_{V P} \mathcal{M}_{V} \\
\mathcal{P}_{2}^{(2)} & \approx \frac{m_{R}}{P_{R 2}^{\beta}} \mathcal{M}_{T}\left[V_{R 2}^{\beta} \mathcal{M}_{T}-P_{R 2}^{\beta} \dot{\mathcal{M}}_{T}+V_{r 2}^{\beta} \mathcal{M}_{V}-P_{r 2}^{\beta} \dot{\mathcal{M}}_{V}\right] \\
& +\frac{m_{R}^{2}}{P_{R 2}^{\beta} P_{R 2}^{\beta}}(E+\mathcal{H})\left[\frac{1}{P_{R 2}^{\beta}} V_{R 2}^{\beta} \mathcal{M}_{T}^{2}+\mathcal{M}_{T} \dot{\mathcal{M}}_{T}\right]
\end{aligned}
$$

and we have denoted

$$
\begin{aligned}
\mathcal{M}_{T} \equiv \frac{\partial R_{2}^{\beta}}{\partial P_{R 1}^{\alpha}}, & \mathcal{M}_{V} \equiv \frac{\partial r_{2}^{\beta}}{\partial P_{R 1}^{\alpha}} \\
\mathcal{M}_{T P} \equiv \frac{\partial P_{R 2}^{\beta}}{\partial P_{R 1}^{\alpha}}, & \mathcal{M}_{V P} \equiv \frac{\partial P_{22}^{\beta}}{\partial P_{R 1}^{\alpha}} \\
V_{R 2}^{\beta} \equiv \frac{\partial V}{\partial R_{2}^{\beta}}, & V_{r 2}^{\beta} \equiv \frac{\partial V}{\partial r_{2}^{\beta}} .
\end{aligned}
$$

The dots on the $\mathcal{M}$ 's indicate time derivative and it is easy to show, using the Jacobi Hamiltonian, that the time evolution is given by

$$
\begin{aligned}
\dot{\mathcal{M}}_{T} & =\frac{1}{m_{R}} \mathcal{M}_{T P} \\
\dot{\mathcal{M}}_{V} & =\frac{1}{m_{r}} \mathcal{M}_{V P} .
\end{aligned}
$$

The derivatives are approximate since we have neglected certain terms involving the Jacobian elements. Note that the first derivative of the phase does not vanish at stationary phase points because we are applying the method only to the translational momentum part of the integrand. This is in contrast to the one dimensional case where the first derivative does vanish at the stationary phase points. Nevertheless, it is interesting to analyze the integrand in Eq. (4.6) as a function of the Filinov parameter $b$.

In the figure (4.6) we show the imaginary part of the integrand for two different representative values of the Filinov parameter $b$. It is clear that with a small value of $b$ it is possible to damp out the PODS singularity around $0.48 \mathrm{eV}$. The main 


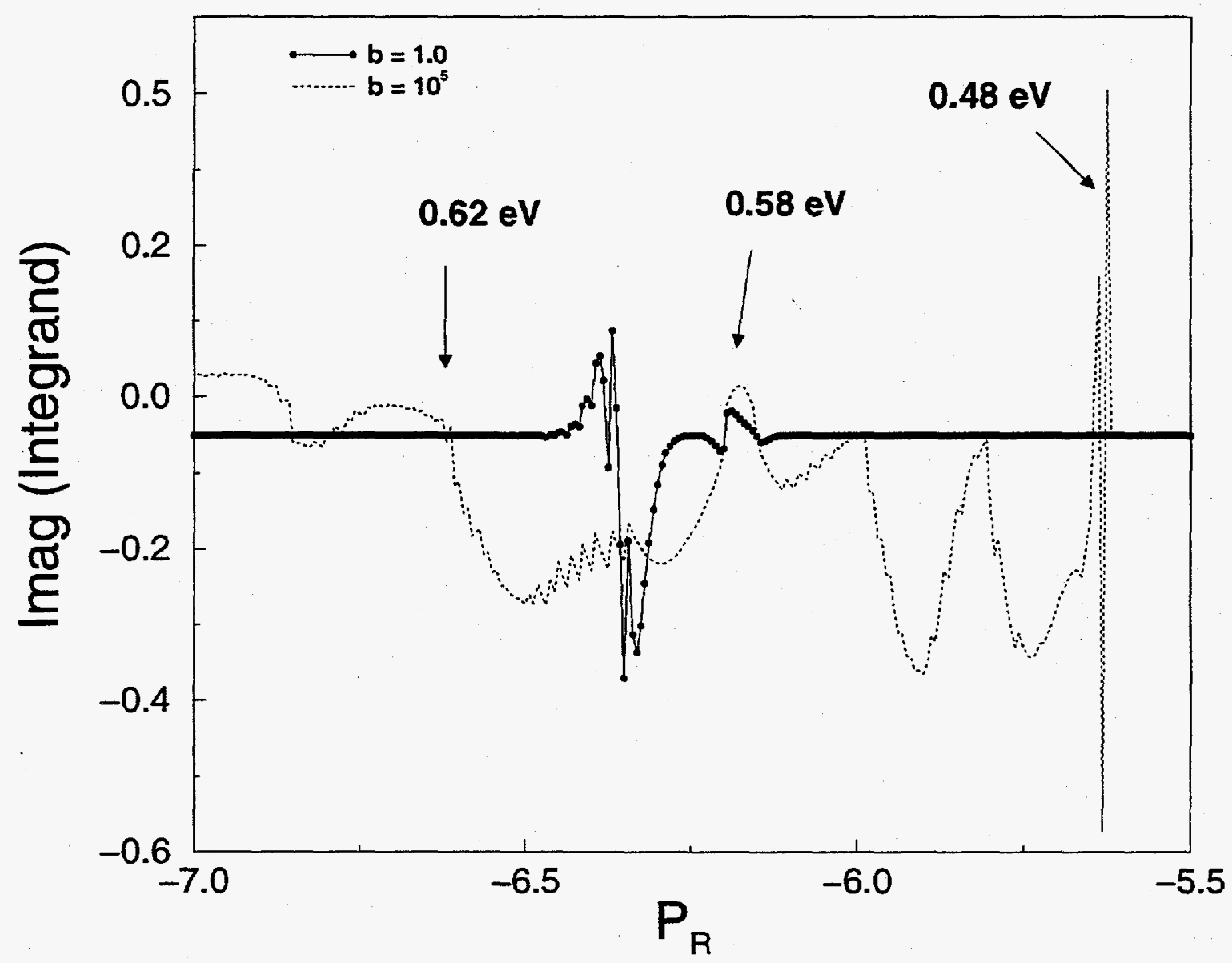

Figure 4.6: This figure shows the imaginary part of the integrand in Eq. (4.6) for two representative values of the Filinov parameter $b$ for $E=0.6 \mathrm{eV}$. Notice the smooth form of the integrand for very small values of $b$ (stationary phase limit) and the PODS singularity structure at $E \approx 0.48 \mathrm{eV}$ emerging for larger values of $b$ (exact limit). The reactive contribution for small values of $b$ is concentrated around the $0.6 \mathrm{eV}$ region. 
contribution for small $b$ is definitely concentrated in the stationary phase region at $E=0.6 \mathrm{eV}$. Even though the integrand looks reasonably smooth as a function of the initial translational momentum, the results still do not compare well with the exact results for collinear $H+H_{2}$. One of the reasons might be that we have not applied the method to the vibrational momentum part of the integrand. It is well known from previous studies that chaotic scattering arises due to the sensitivity of the scattering trajectories to the initial vibrational phase of the diatom. Perhaps treating both the translational and vibrational part of the integrand with the modified Filinov trick would be the correct approach. Another, more pessimistic, reason could be that the complicated nature of these PODS singularities and close proximity of many PODS in a small energy region lead to the failure of this method. It is important to remember that the semiclassical hybrid approach has the best chances of being accurate when the dynamics of interest is inherently of a short time nature. However, reactive trajectories in the neighbourhood of the PODS are relatively long lived and might be a significant factor in the accuracy of the results obtained for this collinear system.

\subsection{Conclusions}

The semiclassical hybrid approach as applied to collinear reactive scattering has a significant numerical bottleneck due to the complicated nature of the integrand. The complications arise due chaotic scattering as a result of which reactive and nonreactive regions are seperated by PODS. We were interested in developing a method which did not rely upon explicitly mapping out the PODS since mapping periodic orbits in greater than two dimensions is prohibitively difficult. The modified Filinov method demonstrated some promise of being successful but application to collinear systems requires a much more careful analysis than presented in this chapter. On the other hand, it is well known from recent work in dynamical systems that chaotic scattering is supressed in greater than two dimensions[81]. The reason being that periodic orbits are one dimensional objects in phase space and clearly in greater than two dimensions will be of less importance for reactive scattering. Thus, it seems 
reasonable to apply the semiclassical hybrid approach to three dimensional systems and test the accuracy of the method. From a numerical standpoint the PODS singularity are the largest bottleneck in implementing the semiclassical approach for collinear reactive scattering. Perhaps it would be useful to explicitly map the PODS for collinear systems and develop a customized integration routine to handle the singularities. Finally, if we have a reactive system which exhibits an interesting and important resonance structure then it becomes necessary to develop efficient methods to handle them within our semiclassical hybrid approach. 


\section{Bibliography}

[1] Tunneling, eds., J. Jortner and B. Pullman, Reidel, Dordrecht 1986.

[2] A. Messiah, Quantum Mechanics, Vol.I North-Holland, Amsterdam 1968; J. J. Sakurai, Modern Quantum Mechanics, Benjamin/Cummings, CA 1985

[3] W. F. Polik, C. B. Moore and W. H. Miller, J. Chem. Phys. 89, 3584 (1988); W. F. Polik, D. R. Guyer, W. H. Miller and C. B. Moore, J. Chem. Phys. 92, 3471 (1990).

[4] Tunneling in Biological Systems, eds., B. Chance, D. C. Devault, H. Frauenfelder, R. A. Marcus, J. R. Schrieffer and N. Sutin, Academic Press 1979.

For importance of tunneling in physics see, for example K. Yamamoto, Prog. Theor. Phys. 91,437 (1994); R. D. Carlitz and D. A. Nicole, Ann. Phys. 164, 411 (1985); P. van Baal and A. Auerbach, Nucl. Phys. B275, 93 (1986).

[5] See for example

J. D. Doll, D. L. Freeman and M. J. Gillan, Chem. Phys. Lett. 143, 277 (1988);

N. Makri, Comput. Phys. Commun. 63, 389 (1991), and references therein.

[6] N. Makri and W. H. Miller, J. Chem. Phys. 87, 5781 (1987); Z. Kotler, A. Nitzan and R. Kosloff, Chem. Phys. Lett. 153, 483 (1988); H. D. Meyer, U. Manthe and L. S. Cederbaum, Chem. Phys. Lett. 165, 73 (1990).

[7] D. G. Truhlar and B. C. Garett, Ann. Rev. Phys. Chem. 35, 159 (1984); G. C. Lynch, D. G. Truhlar and B. C. Garett, J. Chem. Phys. 90, 3102 (1989); R. A. 
Marcus and M. E. Coltrin, J. Chem. Phys. 67, 2609 (1977); C. J. Cerjan, S. Shi and W. H. Miller, J. Chem. Phys. 79, 3336 (1983).

[8] Advances in Classical Trajectory Methods, ed. W. L. Hase 1, JAI press 1992; Theory of Chemical Reaction Dynamics vol.III, ed. M. Baer, CRC Press, Boca Raton, FL 1985; Dynamics of Molecular Collisions, Part B, ed. W. H. Miller, Plenum, NY 1976.

[9] W. H. Miller, Advan. Chem. Phys. 25, 25 (1974); W. H. Miller, Advan. Chem. Phys. 30, 74 (1975).

[10] W. H. Miller, J. Chem. Phys. 62, 1899 (1975); S. Coleman, Phys. Rev. D 15, 2929 (1977); C. G. Callan and S. Coleman, Phys. Rev. D 16, 1762 (1977).

[11] T. F. George and W. H. Miller, J. Chem. Phys. 56, 5722 (1972); T. F. George and W. H. Miller, J. Chem. Phys. 57, 2458 (1972); J. D. Doll, T. F. George and W. H. Miller, J. Chem. Phys. 58, 1343 (1973).

[12] A. O. Caldiera and A. J. Leggett, Ann. Phys. (N.Y.) 149, 374 (1983); S. Coleman, Uses of instantons, in The Whys of Subnuclear Physics, ed. A. Zichichi, Plenum, N.Y., 1979; D. Chandler, Theory of Quantum Processes in Liquids, in Les Houches, Session LI, Liquids, Freezing and Glass Transition, Elsevier, 1991.

[13] N. Fröman and P. O. Fröman, JWKB Approximation, North-Holland, Amsterdam, 1965.

[14] See for example

N. Makri and W. H. Miller, J. Chem. Phys. 91, 4026 (1989); B. A. Waite and W. H. Miller, J. Chem. Phys. 76, 2412 (1982); E. J. Heller and R. C. Brown, J. Chem. Phys. 79, 3336 (1983); J. M. G. Llorente and E. Pollak, Chem. Phys. 120, 37 (1988); R. T. Skodje, D. G. Truhlar and B. C. Garett, J. Phys. Chem. 85, 3019 (1981).

[15] J. C. Tully and R. K. Preston, J. Chem. Phys. 55, 562 (1971). 
[16] N. Makri and W. H. Miller, J. Chem. Phys. 91, 4026 (1989).

[17] S. Keshavamurthy, unpublished results.

[18] E. Bosch, M. Moreno and J. M. Lluch, Chem. Phys. Lett. 196, 73 (1992); E. Bosch, M. Moreno and J. M. Lluch, Chem. Phys. Lett. 159, 99 (1992).

[19] V. I. Arnold, Mathematical Methods of Classical Mechanics, Springer- Verlag, NY, 1989.

[20] S. Keshavamurthy and W. H. Miller, Chem. Phys. Lett. 205, 96 (1993).

[21] W. H. Miller, Faraday Discussions Chem. Soc. 62, 40 (1977).

[22] D. Papoušek and M. R. Aliev, Molecular Vibrational-Rotational Spectra, Elsevier, Amsterdam, 1982.

[23] A. Einstein, Verh. Dtsch. Phys. Ges. 19, 82 (1917); M. L. Brillouin, J. Phys. 7, 353 (1926); J. B. Keller, Ann. Phys. 4, 180 (1958).

[24] M. S. Child, Semiclassical Mechanics with Molecular Applications, Clarendon Press, Oxford, 1991.

[25] W. H. Green, D. Jayatilaka, A. Willetts, R. D. Amos and N. C. Handy, J. Chem. Phys. 93, 4965 (1990); M. J. Cohen, A. Willetts and N. C. Handy, J. Chem. Phys. 99, 5885 (1993).

[26] W. H. Miller, R. Hernandez, C. B. Moore and W. F. Polik, J. Chem. Phys. 93, 5657 (1990); W. H. Miller, R. Hernandez, N. C. Handy, D. Jayatilaka and A. Willetts, Chem. Phys. Lett. 172, 62 (1990); R. Hernandez, W. H. Miller, C. B. Moore and W. F. Polik, J. Chem. Phys. 99, 950 (1993).

[27] J. R. Cary, Phys. Rep. 79, 129 (1981).

[28] E. L. Sibert, J. Chem. Phys. 88, 4378 (1988), and references therein.

[29] E. A. Solv'ev, Soviet Physics JETP 48, 635 (1978); R. T. Skodje, F. Borondo and W. P. Reinhardt, J. Chem. Phys. 82, 4611 (1985). 
[30] A. J. Lichtenberg and M. A. Lieberman, Regular and Stochastic Motion, Springer-Verlag, NY, 1983.

[31] W. L. Hase, D. G. Buckowski and K. N. Swamy, J. Phys. Chem. 87, 2754 (1983).

[32] N. Moiseyev, P. R. Certain and F. Weinhold, Mol. Phys. 36, 1613 (1978).

[33] B. V. Chirikov, Phys. Rep. 52, 263 (1979).

[34] P. A. M. Dirac, The Principles of Quantum Mechanics, Clarendon Press, Oxford, 1967, pp 84-89.

[35] B. A. Waite and W. H. Miller, J. Chem. Phys. 74, 3910 (1981).

[36] W. H. Miller, Chem. Rev. 87, 19 (1987); B. A. Waite and W. H. Miller, J. Chem. Phys. 73, 3713 (1980); Y. Qin and D. L. Thompson, J. Chem. Phys. 100, 6445 (1994).

[37] There has been a lot of effort to extend WKB theory to multidimensional tunneling processes. See for example

M. Wilkinson, Physica 21D, 341 (1986); M. Wilkinson and J. H. Hannay, Physica 27D, 201 (1987); T. Banks, C. M. Bender and T. T. Wu, Phys. Rev. D 8, 3346 (1973); T. Banks and C. M. Bender, Phys. Rev. D 8, 3366 (1973); J. L. Gervais and B. Sakita, Phys. Rev. D 16, 3507 (1977); H. J. De Vega, J. L. Gervais and B. Sakita, Nucl. Phys. B139, 20 (1978).

[38] Atomic and Molecular Beam methods, ed. G. Scoles, Oxford University Press 1988; Proceedings of the International School of Physics "Enrico Fermi", G. Scoles 107, 1988; Y. T. Lee, Science 236, 793 (1987).

[39] P. A. Block, L. G. Pedersen and R. E. Miller, J. Chem. Phys. 98, 3754 (1993); V. S. Letokhov, Nonlinear Laser Chemistry, Springer Series in Chemical Physics 22, Springer-Verlag, NY, 1983; R. B. Bernstein, Chemical Dynamics via Molecular Beam and Laser Techniques, Oxford, NY, 1982. 
[40] W. H. Miller, J. Chem. Phys. 61, 4301 (1974); W. H. Miller, J. Chem. Phys. 63, 1166 (1975); S. Chapman, B. C. Garret and W. H. Miller, J. Chem. Phys. 63, $2710(1975)$.

[41] W. H. Miller in Methods in Computational Molecular Physics, ed. S. Wilson and G. H. F. Diercksen, Plenum Press, NY, 1992 pp 519; W. H. Miller, Annu. Rev. Phys. Chem. 41, 245 (1990) and references therein; D. E. Manolopoulos and D. C. Clary, Ann. Rep. C 86, 95 (1989); G. C. Schatz, Ann. Rev. Phys. Chem. 39, 317 (1988); NATO Advanced Research Workshop on the Theory of Chemical Reaction Dynamics, ed. D. C. Clary 170, Dordrecht 1986; Molecular Collision Dynamics, ed. J. M. Bowman 33, Springer- Verlag 1983.

[42] J. Z. H. Zhang, S. I. Chu and W. H. Miller, J. Chem. Phys 886233 (1988); W. H. Miller and B. M. D. D. J. op de Har, J. Chem. Phys. 86, 8213 (1987).

[43] W. H. Miller, S. D. Schwartz and J. W. Tromp, J. Chem. Phys. 79, 4889 (1983).

[44] T. Seideman and W. H. Miller, J. Chem. Phys. 95, 1768 (1991); T. Seideman and W. H. Miller, J. Chem. Phys. 96, 4412 (1992); T. Seideman and W. H. Miller, J. Chem. Phys. 97, 2499 (1992); W. H. Thompson and W. H. Miller, Chem. Phys. Lett 206123 (1993); U. Manthe and W. H. Miller, J. Chem. Phys. 99 3411 (1993); S. M. Auerbach and W. H. Miller, J. Chem. Phys. 986917 (1993).

[45] D. O. Harris, G. G. Engerholm and W. D. Gwinn, J. Chem. Phys. 43, 1515 (1965); A. S. Dickinson and P. R. Certain, J. Chem. Phys. 49, 4209 (1968); J. C. Light, I. P. Hamilton and J. V. Lill, J. Chem. Phys. 82, 1400 (1985); D. T. Colbert and W. H. Miller, J. Chem. Phys. 96, 1982 (1992).

[46] See for example

R. Kosloff and D. Kosloff, J. Comput. Phys. 63, 363 (1986); C. Leforestier and R. E. Wyatt, J. Chem. Phys. 78, 2334 (1983); D. Neuhauser and M. Baer, J. Chem. Phys. 90, 4351 (1989).

[47] See for example

U. Peskin and W. H. Miller, J. Chem. Phys., to be published. 
[48] G. C. Groenenboom, Report, Netherlands Organization for Scientific Research (NWO), 1992; G. C. Groenenboom and D. T. Colbert, J. Chem. Phys. 99, 9681 (1993).

[49] W. H. Thompson and W. H. Miller, to be published.

[50] U. Manthe, T. Seideman and W. H. Miller, J. Chem. Phys. 101, 4759 (1994); U. Manthe, T. Seideman and W. H. Miller, J. Chem. Phys. 99, 10078 (1993).

[51] G. C. Schatz, M. C. Molton and J. L. Grant, J. Phys. Chem. 88, 2971 (1984); G. C. Schatz, Topics Current Phys. 33, 25 (1983); G. Lendvay and G. C. Schatz, J. Chem. Phys. 98, 1034 (1993).

[52] For example, mode specific effects in the $\mathrm{H}+\mathrm{H}_{2} \mathrm{O}$ reaction was predicted in: H. Elgersma and G. C. Schatz, Int. J. Quant. Chem. Symp. 15, 611 (1981); T. H. Dunning Jr., L. B. Harding, A. F. Wagner, G. C. Schatz and J. M. Bowman, Science 240, 453 (1988).

[53] R. A. Marcus, Ber. Bunsenges. Phys. Chem. 81, 190 (1977); W. L. Hase and D. G. Buckowski, J. Comput. Chem. 3, 335 (1982); G. C. Schatz, J. Chem. Phys. 79, 5386 (1983).

[54] There have been attempts to include the correct zero point energies into classical trajectories in a consistent fashion. However, these are not quite practical and much remains to be understood about the problem. See for example W. H. Miller, W. L. Hase and C. L. Darling, J. Chem. Phys. 91, 2863 (1989); T. D. Sewell, D. L. Thompson, J. D. Gezelter and W. H. Miller, Chem. Phys. Lett. 193, $512(1992)$.

[55] S. Keshavamurthy and W. H. Miller, Chem. Phys. Lett. 218, 189 (1994).

[56] R. B. Gerber and M. A. Ratner, Advan. Chem. Phys. 90, 97 (1988).

[57] This is realted to the fact that the WKB energy eigenfunctions are not exact although the time propagator is exact. 
[58] E. J. Heller, J. Chem. Phys. 94, 2723 (1991); M. Sepúlveda, S. Tomsovic and E. J. Heller, Phys. Rev. Lett. 69, 402 (1992); S. Tomsovic and E. J. Heller, Phys. Rev. E 47, 282 (1993); E. J. Heller, Prog. Theor. Phys. Supp. 116, 45 (1994); E. J. Heller and S. Tomsovic, Physics Today 46, 38 (1993); E. J. Heller in Chaos and Quantum Physics, eds., M. J. Giannoni, A. Voros and J. Zinn-Justin, Elsevier Science, 1991.

[59] M. A. Sepúlveda and E. J. Heller, J. Chem. Phys. 101, 8004 (1994).

[60] For a rigorous introduction to Maslov indicies see

V. P. Maslov and M. V. Fedoriuk, Semiclassical Approximations in Quantum Mechanics, Reidel, Dordrecht, 1981; J. M. Robbins, Studies in Semiclassical Quantization, Ph.D. thesis, U. C. Berkeley, 1983.

[61] For a more detailed discussion of the S-matrix version of the Kohn variational principle, see

L. F. Gaucher, A Discrete Variable Representation for Electron-Hydrogen Atom Scattering, Ph.D. thesis, U. C. Berkeley, 1993.

[62] R. P. Feynman and A. R. Hibbs, Quantum Mechanics and Path Integrals, McGraw-Hill, NY, 1965.

[63] P. Pechukas, Phys. Rev. 181, 166 (1969); D. Gelman and L. Spruch, J. Math. Phys. 10, 2240 (1969); A. P. Penner and R. Wallace, Phys. Rev. A 7, 1007 (1973); W. H. Miller, J. Chem. Phys. 53, 1949 (1970); W. H. Miller, J. Chem. Phys. 53, 3578 (1970).

[64] J. H. Van Vleck, Proc. Natl. Acad. Sci. 14, 178 (1928); M. C. Gutzwiller, J. Math. Phys. 8, 1979 (1967).

[65] C. Eckart, Phys. Rev. 35, 1303 (1930).

[66] H. S. Johnston, Gas Phase Reaction Rate Theory, Ronald, NY, 1966 p. 44. 
[67] See for example

L. D. Landau and E. M. Lifshitz, Quantum Mechanics, Pergammon, Oxford, 1958, chap 7; M. V. Berry and K. E. Mount, Rep. Prog. Phys. 35, 315 (1972).

[68] M. V. Berry, N. L. Balazs, M. Tabor and A. Voros, Ann. Phys. (N.Y.) 122, 26 (1979).

[69] B. W. Spath and W. H. Miller, to be published.

[70] See for example

B. Eckhardt, Physica D 33, 89 (1988); J. H. Jensen, Phys. Rev. Lett. 73, 244 (1994).

[71] Please see appendix I of the previous chapter and the references therein for a detailed discussion of this observation.

[72] R. B. Dingle, Asymptotic expansions: their derivation and interpretation, Academic Press, NY, 1973.

[73] H. Weyl, Z. Phys. 46, 1 (1927); J. E. Moyal, Proc. Camb. Phil. Soc. 45, 99 (1949).

[74] D. E. Makarov and N. Makri, Chem. Phys. Let. 221, 482 (1994); N. Makri, Chem. Phys. Let. 193, 435 (1992); N. Makri, J. Chem. Phys. 94, 4949 (1991).

[75] D. G. Truhlar and C. J. Horowitz, J. Chem. Phys. 68, 2466 (1978); D. G. Truhlar and C. J. Horowitz, J. Chem. Phys. 71, 1514(E) (1979); P. Seigbahn and B. Liu, J. Chem. Phys. 68, 2457 (1978).

[76] S. Keshavamurthy and W. H. Miller, unpublished notes.

[77] See for example

L. Pauling and E. B. Wilson, Jr., Introduction to Quantum Mechanics, Dover, NY, 1963, pp 271-274.

[78] M. Abramowitz and I. A. Stegun, Handbook of Mathematical Functions with Formulas, Graphs and Mathematical Tables, Wiley, NY, 1972. 
[79] M. J. Davis, J. Chem. Phys. 86, 3978 (1987); R. T. Skodje and M. J. Davis, J. Chem. Phys. 88, 2429 (1988); M. J. Davis, J. Chem. Phys. 83, 1016 (1985); M. J. Davis and S. K. Gray, J. Chem. Phys. 84, 5389 (1986).

[80] E. Pollak and P. Pechukas, J. Chem. Phys. 70, 325 (1979); E. Pollak in Theory of Chemical Reaction Dynamics, Ed., M. Baer, CRC Press, Boca Reaton, FL, 1985, Vol. III, p 65.

[81] See for example S. Mikkola, Month. Not. Roy. Ast. Soc. 269, 127 (1994); B. B. Grayce, R. T. Skodje and J. M. Hutson, J. Chem. Phys. 98, 3929 (1993); M. Ding, C. Grebogi, E. Ott and J. A. Yorke, Phys. Rev. A 42, 7025 (1990); R. Blumel and U. Smilansky, Phys. Rev. Lett. 64, 241 (1990).

[82] N. Makri and W. H. Miller, Chem. Phys. Lett. 139, 10 (1987); V. S. Filinov, Nucl. Phys. B 271, 717 (1986).

[83] For more detailed analysis of the modified Filinov method see N. Makri, Theoretical Methods for the Study of Chemical Dynamics, Ph.D. thesis, U. C. Berkeley, 1989.

[84] G. R. Fleming and A. E. Siegman, Eds., Ultrafast Phenomena V, Springer-Verlag, NY, 1986; C. V. Shank, Science 233, 1276 (1986); A. H. Zewail and R. B. Bernstein, Chem.\& Eng. News 66, 24 (1988); M. J. Rosker, M. Dantus and A. H. Zewail, Science 241, 200 (1988); S. Mukamel, in Ultrafast Phenomena VI, Springer-Verlag, NY, 1988.

[85] R. P. Feynman, Statistical Mechanics, Benjamin, Reading, 1972; R. P. Feynman, Phys. Rev. 97, 660 (1955); D. Chandler and P. G. Wolynes, J. Chem. Phys. 74, 4079 (1981); R. P. Feynman and F. L. Vernon, Annal. Phys. 24, 118 (1963).

[86] M. P. Allen and D. J. Tildesley, Computer Simulations of Liquids, Oxford University Press, Oxford, 1987. 
[87] A. M. Ozorio de Almeida, Hamiltonian Systems: Chaos and Quantization, Cambridge University Press, 1988.

[88] M. Tabor, Chaos and Integrability in Nonlinear Dynamics. An Introduction, Wiley, NY, 1989.

[89] M. C. Gutzwiller, Chaos in Classical and Quantum Mechanics, Springer-Verlag, NY, 1990.

[90] O. Bohigas, S. Tomsovic and D. Ullmo, Phys. Rep. 223, 43 (1993).

[91] In general, analytically continuing classical mechanics leads to complex phase space and there are questions about the smoothness of the manifolds in phase space and other subtleties. There is no rigorous theory concerning these issues and is still an open problem. Anyhow, even after doing analytical continuation we have to build in quantum superposition.

[92] S. Kovalevskaya, Acta Math. 12, 177 (1889); M. Tabor, Nature 310, 277 (1984); H. Yoshida, Celest. Mech. 31, 363 (1983); Y. F. Chang, J. M. Greene, M. Tabor and J. Weiss, Physica D 8, 183 (1983).

[93] R. G. Littlejohn, J. Stat. Phys. 68, 7 (1992).

[94] The Physics of Phase Space, Ed. Y. S. Kim and W. W. Zachary, Springer-Verlag, NY, 1986.

[95] M. C. Gutzwiller, J. Math. Phys. 8, 1979 (1967).

[96] R. Artuso, E. Aurell and P. Cvitanović, Nonlinearity 3, 325, 361 (1990).

[97] R. G. Littlejohn, J. Math. Phys. 31, 2952 (1990); S. C. Creagh, J. M. Robbins and R. G. Littlejohn, Phys. Rev. A 42, 1907 (1990); S. C. Creagh and R. G. Littlejohn, J. Phys. A: Math. Gen. 25, 1643 (1992).

[98] B. Eckhardt and D. Wintgen, J. Phys. B: At. Mol. Opt. Phys. 23, 355 (1990); H. Friedrich and D. Wintgen, Phys. Rep. 183, 37 (1989); G. S. Ezra, K. Richter, G. Tanner and D. Wintgen, J. Phys. B: At. Mol. Opt. Phys. 24, 413 (1991). 
[99] M. V. Berry and J. P. Keating, J. Phys. A: Mat. Gen. 23, 4839 (1990).

[100] K. W. Ford and J. A. Wheeler, Ann. Phys. N.Y., 7, 259, 287 (1959). 